Prepared in cooperation with the Washington County Water Conservancy District

\title{
Assessment of Managed Aquifer Recharge at Sand Hollow Reservoir, Washington County, Utah, Updated to Conditions in 2012
}

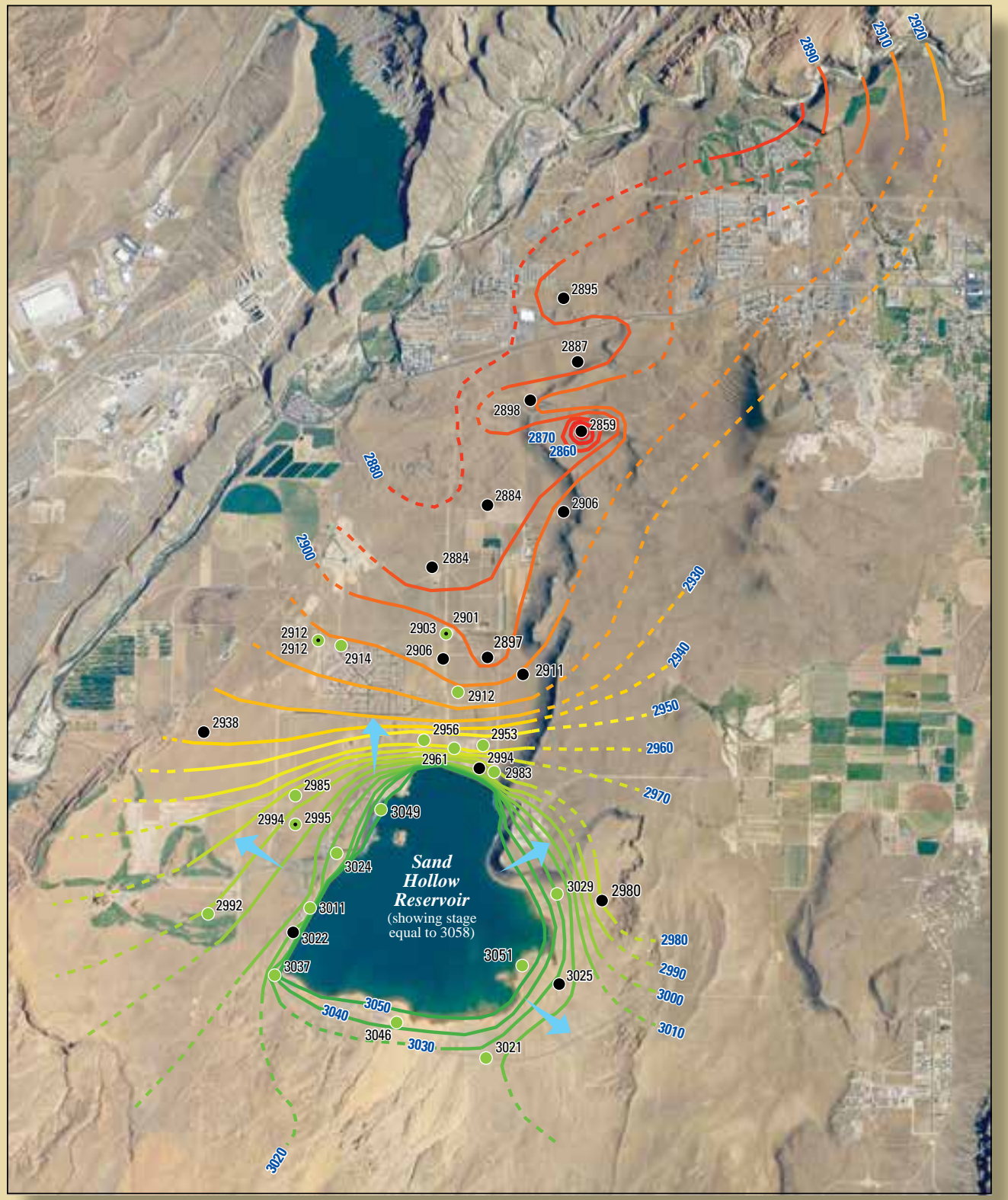

Scientific Investigations Report 2013-5057

U.S. Department of the Interior

U.S. Geological Survey 



\section{Assessment of Managed Aquifer Recharge at Sand Hollow Reservoir, Washington County, Utah, Updated to Conditions in 2012}

By Thomas M. Marston and Victor M. Heilweil

Prepared in cooperation with the Washington County Water Conservancy District

Scientific Investigations Report 2013-5057 


\section{U.S. Department of the Interior \\ KEN SALAZAR, Secretary}

\section{U.S. Geological Survey \\ Suzette M. Kimball, Acting Director}

U.S. Geological Survey, Reston, Virginia: 2013

For more information on the USGS - the Federal source for science about the Earth, its natural and living resources, natural hazards, and the environment, visit http://www.usgs.gov or call 1-888-ASK-USGS.

For an overview of USGS information products, including maps, imagery, and publications, visit http://www.usgs.gov/pubprod

To order this and other USGS information products, visit http://store.usgs.gov

Any use of trade, firm, or product names is for descriptive purposes only and does not imply endorsement by the U.S. Government.

Although this information product, for the most part, is in the public domain, it also may contain copyrighted materials as noted in the text. Permission to reproduce copyrighted items must be secured from the copyright owner.

Suggested citation:

Marston, T.M. and Heilweil, V.M., 2013, Assessment of managed aquifer recharge at Sand Hollow Reservoir, Washington County, Utah, updated to conditions in 2012: U.S. Geological Survey Scientific Investigations Report 2013-5057, 43 p. 


\section{Contents}

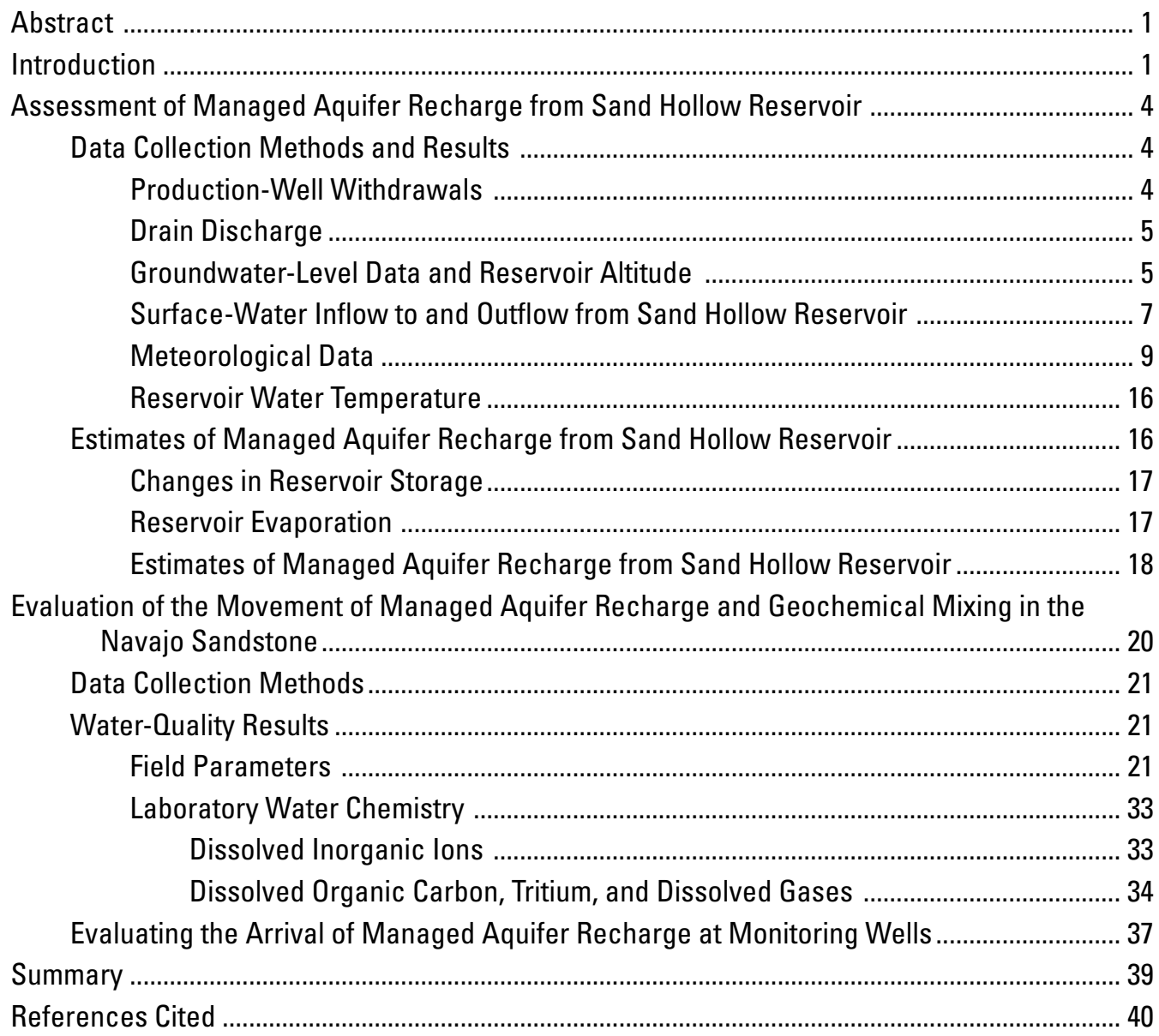

\section{Figures}

1. Map showing location of the Sand Hollow study area, Washington County, Utah .............. 2

2. Map showing location of wells, the weather station, drains, and reservoir sampling sites in Sand Hollow, Utah ........................................................................................ 3

3. Graph showing Washington County Water Conservancy District production-well withdrawals in Sand Hollow, Utah, 2004-11 ..................................................................... 4

4. Graph showing monthly reported discharge from the North Dam drain, West Dam drain, and West Dam Spring drain in Sand Hollow, Utah, 2003-11 ................................ 5

5. Graph showing water-level altitude in selected wells and Sand Hollow Reservoir, Sand Hollow, Utah, 1995-2011 ............................................................................... 6

6. Map showing potentiometric surface of the Navajo aquifer in January 2012, Sand

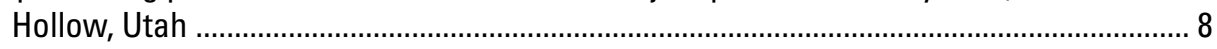

7. Graph showing monthly precipitation at Sand Hollow, Utah, 1998-2011 ............................. 9

8. Graph showing daily water temperature at various depths in Sand Hollow Reservoir,

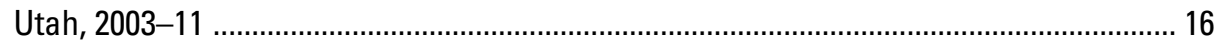


9. Graph showing monthly estimated evaporation, recharge, and reservoir altitude, Sand Hollow Reservoir, Utah, 2002-11

10. Graph showing monthly calculated recharge rates beneath Sand Hollow Reservoir, Utah, 2002-11

11. Graph showing estimated annual inflow, evaporation, and recharge from Sand Hollow Reservoir, Utah, 2002-11

12. Graph showing total dissolved-gas pressure in groundwater from selected monitoring wells in Sand Hollow, Utah, 2001-2012

13. Graphs showing specific conductance of reservoir water and groundwater from selected monitoring wells in Sand Hollow, Utah, 1999-2012

14. Graph showing major-ion chemistry from selected surface water and groundwater sites in Sand Hollow, Utah

15. Graph showing chloride-to-bromide ratios of reservoir water and groundwater from selected monitoring wells in Sand Hollow, Utah, 2001-12

16. Map showing tritium concentrations of reservoir water and groundwater from selected monitoring wells in Sand Hollow, Utah, February 2012

17. Map showing CFC-12 concentrations of reservoir water and groundwater from selected monitoring wells in Sand Hollow, Utah, February 2012

\section{Tables}

1. Reservoir data, evaporation, and calculated recharge from Sand Hollow Reservoir, Utah, 2002-11

2. Field water-quality parameters, dissolved organic carbon, tritium, chlorofluorocarbons, sulfur hexafluoride, and neon excess in groundwater and surface water from Sand Hollow, Utah

3. Major and minor chemical constituents in groundwater and surface water from selected sites in Sand Hollow, Utah

4. Summary of tracer peaks showing year of arrival for reservoir recharge at selected monitoring wells in Sand Hollow, Utah 


\section{Conversion Factors, Datums, and Abbreviated Water-Quality Units}

Inch/Pound to SI

\begin{tabular}{|c|c|c|}
\hline Multiply & By & To obtain \\
\hline \multicolumn{3}{|c|}{ Length } \\
\hline inch (in) & 2.54 & centimeter $(\mathrm{cm})$ \\
\hline foot $(\mathrm{ft})$ & 0.3048 & meter $(\mathrm{m})$ \\
\hline mile (mi) & 1.609 & kilometer (km) \\
\hline \multicolumn{3}{|c|}{ Area } \\
\hline acre & 4,047 & square meter $\left(\mathrm{m}^{2}\right)$ \\
\hline acre & 0.004047 & square kilometer $\left(\mathrm{km}^{2}\right)$ \\
\hline square foot $\left(\mathrm{ft}^{2}\right)$ & 929.0 & square centimeter $\left(\mathrm{cm}^{2}\right)$ \\
\hline square foot $\left(\mathrm{ft}^{2}\right)$ & 0.09290 & square meter $\left(\mathrm{m}^{2}\right)$ \\
\hline square inch (in²) & 6.452 & square centimeter $\left(\mathrm{cm}^{2}\right)$ \\
\hline square mile $\left(\mathrm{mi}^{2}\right)$ & 2.590 & square kilometer $\left(\mathrm{km}^{2}\right)$ \\
\hline \multicolumn{3}{|c|}{ Volume } \\
\hline gallon (gal) & 3.785 & liter (L) \\
\hline cubic foot $\left(\mathrm{ft}^{3}\right)$ & 0.02832 & cubic meter $\left(\mathrm{m}^{3}\right)$ \\
\hline acre-foot (acre-ft) & 1,233 & cubic meter $\left(\mathrm{m}^{3}\right)$ \\
\hline \multicolumn{3}{|c|}{ Flow rate } \\
\hline acre-foot per day (acre-ft/d) & 0.01427 & cubic meter per second (m³/s) \\
\hline \multicolumn{3}{|c|}{ Mass } \\
\hline pound, avoirdupois (Ib) & 0.4536 & kilogram (kg) \\
\hline \multicolumn{3}{|c|}{ Density } \\
\hline pound per cubic foot (lb/ft³) & 16.02 & kilogram per cubic meter $\left(\mathrm{kg} / \mathrm{m}^{3}\right)$ \\
\hline \multicolumn{3}{|c|}{ Hydraulic conductivity } \\
\hline foot per day (ft/d) & 0.3048 & meter per day $(\mathrm{m} / \mathrm{d})$ \\
\hline
\end{tabular}

Temperature in degrees Celsius $\left({ }^{\circ} \mathrm{C}\right)$ may be converted to degrees Fahrenheit ( $\left.{ }^{\circ} \mathrm{F}\right)$ as follows:

${ }^{\circ} \mathrm{F}=\left(1.8 \times{ }^{\circ} \mathrm{C}\right)+32$

Temperature in degrees Fahrenheit $\left({ }^{\circ} \mathrm{F}\right)$ may be converted to degrees Celsius $\left({ }^{\circ} \mathrm{C}\right)$ as follows:

${ }^{\circ} \mathrm{C}=\left({ }^{\circ} \mathrm{F}-32\right) / 1.8$

Vertical coordinate information is referenced to the North American Vertical Datum of 1988 (NAVD 88). Horizontal coordinate information is referenced to the North American Datum of 1983 (NAD 83).

Altitude, as used in this report, refers to distance above the vertical datum.

Specific conductance is given in microsiemens per centimeter at 25 degrees Celsius $\left(\mu \mathrm{S} / \mathrm{cm}\right.$ at $\left.25^{\circ} \mathrm{C}\right)$.

Concentrations of chemical constituents in water are given either in milligrams per liter $(\mathrm{mg} / \mathrm{L})$ or micrograms per liter $(\mu \mathrm{g} / \mathrm{L})$.

Stable isotope concentrations are reported as per mil, which is equivalent to parts per thousand.

Tritium units (TU) are used to report tritium concentration, where one TU equals tritium concentration in picoCuries per liter divided by 3.22 .

Chlorofluorocarbon concentrations are reported as picomoles per kilogram (pmol/kg).

Sulfur hexafluoride concentrations are reported as femtomoles per kilogram (fmol/kg). 


\section{Acronyms and Abbreviations}

$\begin{array}{ll}\text { CFCs } & \text { chlorofluorocarbons } \\ \text { CI } & \text { chloride } \\ \text { Cl:Br } & \text { chloride to bromide ratio } \\ \text { DO } & \text { dissolved oxygen } \\ \text { DOC } & \text { dissolved organic carbon } \\ \mathrm{R}_{2} & \text { coefficient of determination } \\ \text { SF }_{6} & \text { sulfur hexafluoride } \\ \text { SHSP } & \text { Sand Hollow State Park } \\ \text { TDG } & \text { total dissolved gas } \\ \text { USGS } & \text { U.S. Geological Survey } \\ \text { WCWCD } & \text { Washington County Water Conservancy District }\end{array}$




\title{
Assessment of Managed Aquifer Recharge at Sand Hollow Reservoir, Washington County, Utah, Updated to Conditions in 2012
}

\author{
By Thomas M. Marston and Victor M. Heilweil
}

\section{Abstract}

Sand Hollow Reservoir in Washington County, U tah, was completed in M arch 2002 and is operated primarily for managed aquifer recharge by the Washington County Water Conservancy District. From 2002 through 2011, surfacewater diversions of about 199,000 acre-feet to Sand Hollow Reservoir have allowed the reservoir to remain nearly full since 2006. Groundwater levels in monitoring wells near the reservoir rose through 2006 and have fluctuated more recently because of variations in reservoir altitude and nearby pumping from production wells. B etween 2004 and 2011, a total of about 19,000 acre-feet of groundwater was withdrawn by these wells for municipal supply. In addition, a total of about 21,000 acre-feet of shallow seepage was captured by French drains adjacent to the North and West Dams and used for municipal supply, irrigation, or returned to the reservoir.

From 2002 through 2011, about 106,000 acre-feet of water seeped beneath the reservoir to recharge the underlying Navajo Sandstone aquifer. Water quality was sampled at various monitoring wells in Sand Hollow to evaluate the timing and location of reservoir recharge as it moved through the aquifer. Tracers of reservoir recharge include major and minor dissolved inorganic ions, tritium, dissolved organic carbon, chlorofluorocarbons, sulfur hexafluoride, and noble gases. By 2012, this recharge arrived at four monitoring wells located within about 1,000 feet of the reservoir. Changing geochemical conditions at five other monitoring wells could indicate other processes, such as changing groundwater levels and mobilization of vadose-zone salts, rather than arrival of reservoir recharge.

\section{Introduction}

Sand Hollow Reservoir (fig. 1) in Washington County, U tah, was completed in M arch 2002 and is operated primarily for managed aquifer recharge by the Washington County Water Conservancy District. The reservoir is an off-channel facility that receives water diverted from the Virgin River near the town of Virgin, Utah. Sand Hollow has been the subject of interdisciplinary, cooperative investigations of groundwater hydrology and geochemistry since 1999. Previous Sand Hollow reports document pre-reservoir vadose-zone and groundwater conditions prior to $\mathrm{M}$ arch 2002 (Heilweil and Solomon, 2004; Heilweil and others, 2006; Heilweil and others, 2007; Heilweil and M CK inney, 2007; Heilweil and others, 2009b), pond and trench infiltration studies adjacent to the reservoir (Heilweil and others, 2004; Heilweil and Watt, 2011), and post-reservoir groundwater conditions, water budgets, and estimates of groundwater recharge from the reservoir from $M$ arch 2002 through December 2009 (Heilweil and others, 2005; Heilweil and Susong, 2007; Heilweil and others, 2009a; Heilw eil and $M$ arston, 2011). These reports al so contain monitoring-well and production-well completion information, as well as historical water-quality and precipitation data. The objectives of this report are to present and interpret (1) groundwater levels, reservoir altitude, well withdrawals, drain discharge, meteorological data, reservoir water temperature, and inflows/outflows from March 2002 through December 2011 for estimating monthly amounts of managed aquifer recharge from Sand Hollow Reservoir to the Navajo Sandstone, and (2) groundwater and surface water chemical data collected prior to the construction of the reservoir through March 2012 for evaluating groundwater flow paths and travel times of this managed aquifer recharge. This study is a cooperative effort by the Washington County Water Conservancy District (W CW CD) and the U.S. Geological Survey (USGS). Support for this work was provided by both the USGS and the WCWCD.

From 2002 through 2011, total surface-water diversions of about 199,000 acre-ft to Sand Hollow Reservoir have allowed the reservoir to remain nearly full since 2006. G roundwater levels in monitoring wells near the reservoir rose through 2006 and then fluctuated more recently because of variations in reservoir water-level altitude and nearby pumping from production wells. B etween 2004 and 2011, a total of about 19,000 acre-ft of groundwater was withdrawn by these wells for municipal supply. In addition, a total of about 21,000 acre-ft of shallow seepage was captured by French drains adjacent to the North and West Dams (fig. 2) and used for municipal supply, irrigation, or returned to the reservoir. 


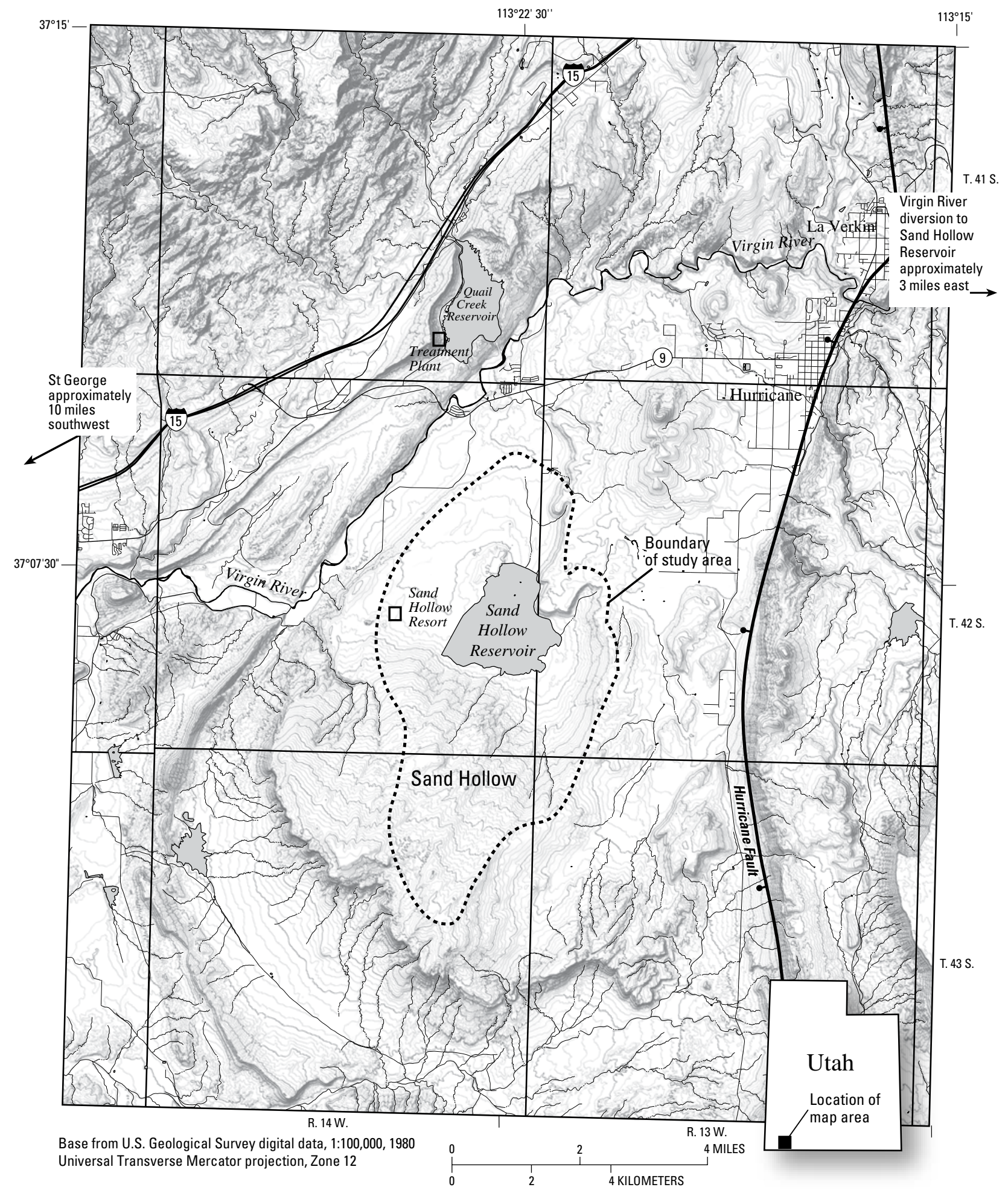

Figure 1. Location of the Sand Hollow study area, Washington County, Utah. 


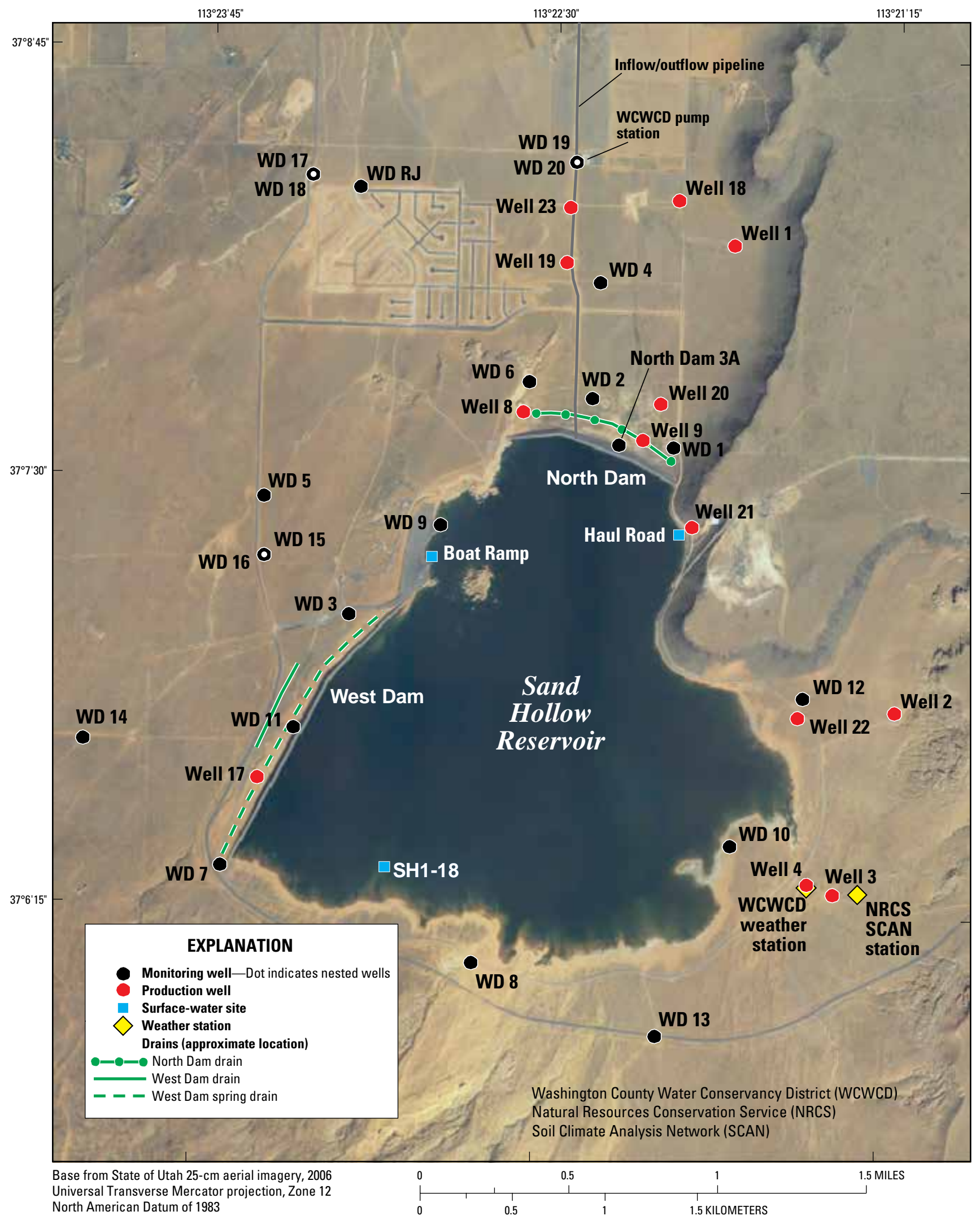

Figure 2. Location of wells, the weather station, drains, and reservoir sampling sites in Sand Hollow, Utah. 


\section{Assessment of Managed Aquifer Recharge from Sand Hollow Reservoir}

$M$ any different types of data have been collected to investigate recharge processes, to quantify recharge from Sand Hollow Reservoir, and to evaluate hydraulic and geochemical changes in the underlying Navajo Sandstone aquifer. These data include production-well withdrawals near the reservoir, amounts of pumpage from drains capturing shallow groundwater discharge adjacent to the reservoir, reservoir and monitoring-well water levels, meteorological parameters, reservoir water temperatures, and inflows and outflows through the pipeline connecting Sand Hollow Reservoir with the Virgin River and Quail Creek Reservoir and treatment plant (fig. 1).

\section{Data Collection Methods and Results}

Data collection methods are described in detail in Heilweil and others (2005) and briefly summarized in the following sections.

\section{Production-Well Withdrawals}

The W CW CD has 13 production wells completed in the Navajo Sandstone available to capture both pre-existing groundwater (natural recharge) in Sand Hollow and recharge from Sand Hollow Reservoir (fig. 2). The WCWCD and other water users have withdrawn natural recharge in Sand Hollow for many years. The W CW CD groundwater withdrawals are recorded monthly from in-line magnetic flow meters installed at each well. Since A ugust 2004, monthly withdrawals by the WCWCD have generally exceeded 150 acre-ft, except for several months during the winters of 2004-05, 2005-06, and 2008-09 (fig. 3). The majority of this pumping has been from Wells 8 and 9 (fig. 2), both located adjacent to the North Dam. From 2004 through 2006, there were minimal withdrawals from these wells during the winter. Since 2006, withdraw als have been more constant year-round. Combined monthly withdrawals from these two production wells averaged about 220 acre-ft from M arch 2006 through December 2011. Greater amounts of pumping from Well 19 began during the summer of 2010 and remained near constant through 2011 at about 70 acre-ft per month. Smaller amounts have been withdrawn from Wells 1, 2, 17, and 21. A total of about 19,000 acre-ft were pumped from the WCWCD production wells from J anuary 2004 through December 2011. Through 2011, withdrawals by the W CW CD at Sand Hollow have been permitted by the U tah Division of Water Rights as natural recharge in Sand Hollow. These withdrawals are governed by different water rights than recharge from Sand Hollow Reservoir; withdrawal rights for this artificial recharge have not yet been exercised.

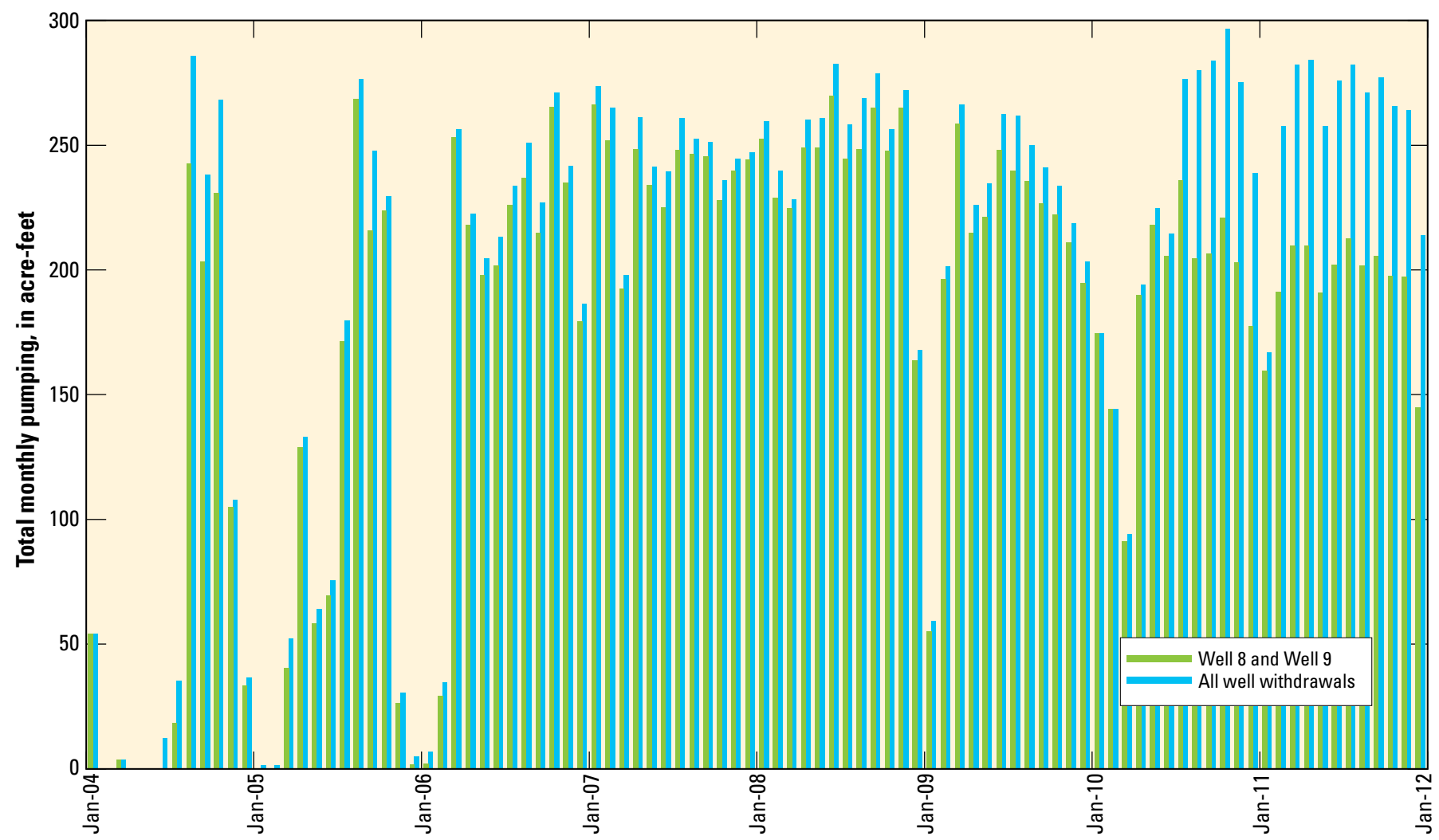

Figure 3. Washington County Water Conservancy District production-well withdrawals in Sand Hollow, Utah, $2004-11$. 


\section{Drain Discharge}

B ecause of the steep gradients associated with the hydraulic connection between the reservoir and the underlying Navajo aquifer, some land-surface areas downgradient of the N orth and West Dams became saturated following construction of the reservoir. In response, three French drains (N orth Dam drain, West Dam drain, and West Dam Spring drain) were constructed for capturing this shallow groundwater (fig. 2). Timing of excavation and spatial dimensions for the three drains can be found in M arston and Heilweil (2011).

A mounts of discharge pumped from these drains are measured with a Sparling Instruments Tigermag totalizing flow meter. Discharge to the N orth Dam drain has been pumped relatively consistently since September 2003 (fig. 4). About 6,000 acre-ft were pumped from the N orth $\mathrm{Dam}$ drain between 2003 and 2011. Initially, all of this water was returned to the reservoir, but since 2007, the majority of water, along with an additional 5,800 acre-ft of outflow from Sand Hollow Reservoir, has been used by Sand Hollow Resort (fig. 1) to meet irrigation demands. A bout 1,500 acre-ft of water were pumped from the West $D$ am drain back into the reservoir from
2005 through 2011. B eginning in October 2006, pumping of discharge from the West Dam Spring drain was initiated and largely has replaced the need for pumping of the West Dam drain; from 2006 through 2011, about 13,500 acre-ft were pumped from the West Dam Spring drain for municipal use. While drainage to the West Dam Spring drain likely does not vary greatly, pumping from this drain has been intermittent. The intermittent pumping schedule results in high variability in the monthly reported discharge from the West Dam Spring drain (fig. 4).

\section{Groundwater-Level Data and Reservoir Altitude}

Groundwater levels measured in an extensive monitoringwell network surrounding Sand Hollow Reservoir were used to document changes in the potentiometric surface associated with recharge from Sand Hollow Reservoir. The WCWCD measures water levels monthly in 21 monitoring wells completed in the Navajo Sandstone (fig. 2). These wells were constructed with either 1- or 2-in diameter pvc casing, with perforations along the bottom 5 - to $20-\mathrm{ft}$ length of the casing.

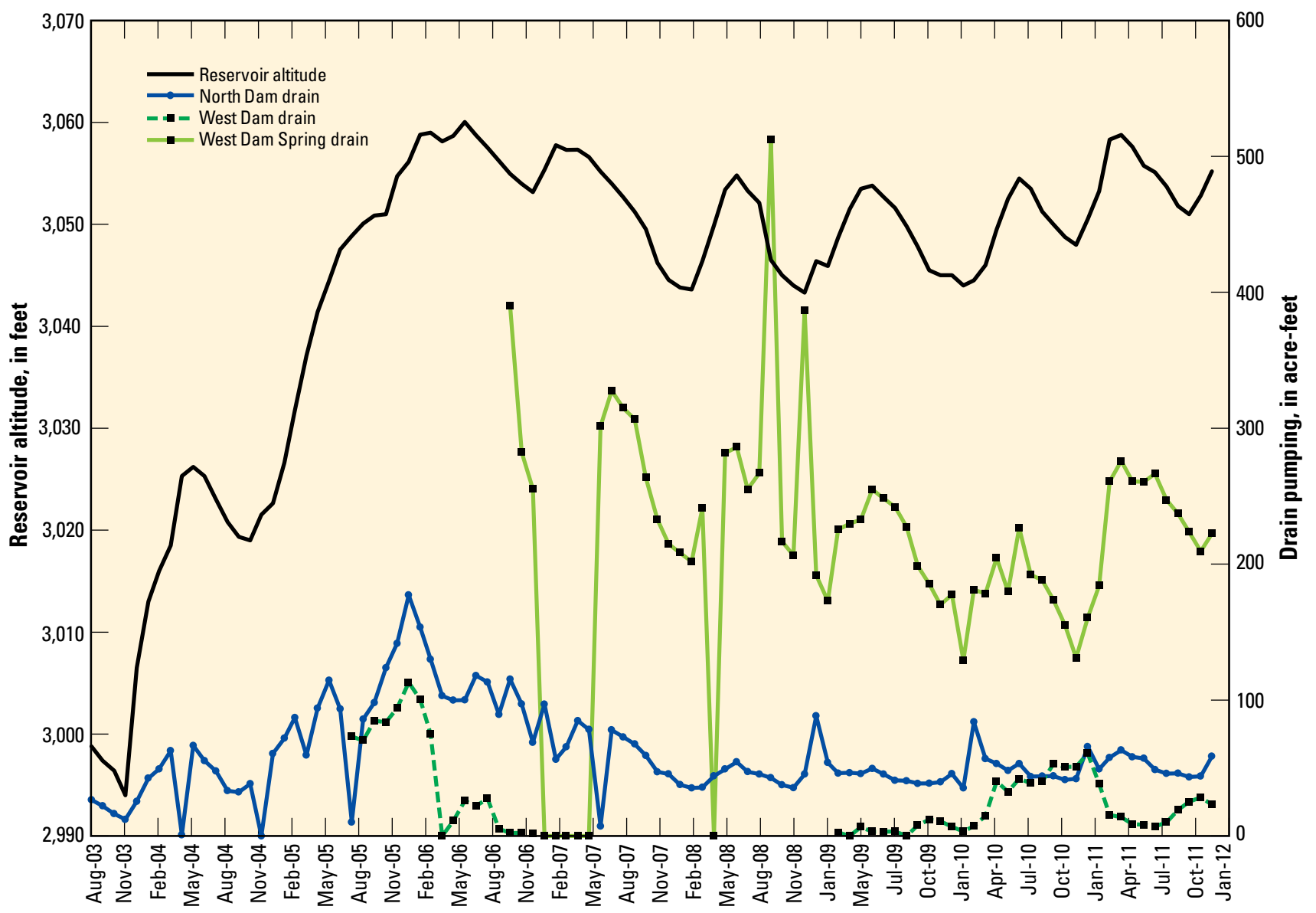

Figure 4. Monthly reported discharge from the North Dam drain, West Dam drain, and West Dam Spring drain in Sand Hollow, Utah, 2003-11. 
Three locations have nested pairs of water district (WD) monitoring wells: WD 15 and WD 16, WD 17 and WD 18, and WD 19 and WD 20. The vertical distance between well screens for the nested-pair wells are $243 \mathrm{ft}, 79 \mathrm{ft}$, and $227 \mathrm{ft}$, respectively. Wells measured by the WCW CD had annual independent check measurements performed by USGS personnel for quality assurance to ensure accuracy of equipment used to measure water levels (electric-tape water-level indicators).

Daily reservoir water-level altitude was recorded from J anuary 2005 through December 2011 by using a pressure transducer installed by the WCW CD in the reservoir along the N orth Dam. B ecause of periods of transducer malfunction from 2005 through 2007, and from A ugust 2011 to December 2011, daily reservoir al titude was interpolated on the basis of monthly measurements recorded at the boat ramp by W CW CD and Sand Hollow State Park (SH SP) personnel, and then correlated with trends from the transducer data. From 2008 through mid-2011, the transducer data were within $0.25 \mathrm{ft}$ of the intermittent boat ramp measurements; thus, for this more recent period, the daily reservoir altitude recorded by the pressure transducer was used.

Recently measured (J anuary 2008 through December 2011) and previously reported (Heilweil and others, 2005; H eilweil and Susong, 2007; Heilweil and others, 2009; Heilweil and $M$ arston, 2011) groundwater levels and reservoir water-level altitude are shown in figure 5 . The reservoir altitude rose from about 2,980 ft at the beginning of March 2002 to a maximum of about 3,060 ft in May 2006, when the reservoir was first filled to capacity. The reservoir altitude receded to about $3,040 \mathrm{ft}$ in December 2007 as a result of reduced inflows and evaporative losses, and then fluctuated between about 3,040 and 3,060 ft from 2008 through 2011. The topographically lowest part of the reservoir bottom, near the N orth Dam, was the first region to be inundated with surface water in 2002 and 2003. Therefore, the monitoring wells nearest the northern side of the reservoir were the first to show water-level responses and hydraulic connection with the reservoir. As the reservoir continued to fill from 2004 through 2006, the extent of stored water increased toward the south, roughly perpendicular to the N orth Dam. Water levels in WD 1, 2, 6, and 9 rose rapidly beginning in the spring of 2002. Water levels in WD 3 and WD 11, located farther south along the western side of the reservoir, began to rise rapidly in N ovember 2002 and J anuary 2003, respectively. Water levels in WD 10 and W D 12 (located on the eastern side of the reservoir) and in WD 7 and WD 8 (located along the southern side of the reservoir) began rising in the latter half of 2003. From 2004 through 2011, measured water levels in monitoring wells closer to the reservoir (WD 3, 7, 8, 9, 10,11,12) generally fluctuated with reservoir altitude. Exceptions to this were water levels in the monitoring wells near the N orth Dam (WD 1,2,6), which all had sharp rises during the winters of 2003-04, 2004-05, 2005-06, 2008-09, 2009-10, and 2010-11 associated with the temporary cessation of pumping at nearby wells 8 and 9 . Drawdown induced by pumping Well 19 from mid-2010 through 2011 is apparent in monitoring wells located farther north of the N orth Dam (WD 4, 19, 20). A t monitoring wells farther from the reservoir (WD 4, 5, RJ , 13, 14), water levels generally displayed a more subdued rise in response to recharge beneath the reservoir. Water levels have been measured only since M ay 2009 in WD 15, 16, 17, 18, 19, and 20. Changes in water levels from those observed in 1995 (Heilweil and others, 2000) indicate that much of the drawdown induced by irrigation pumping to the north of the reservoir has recovered.

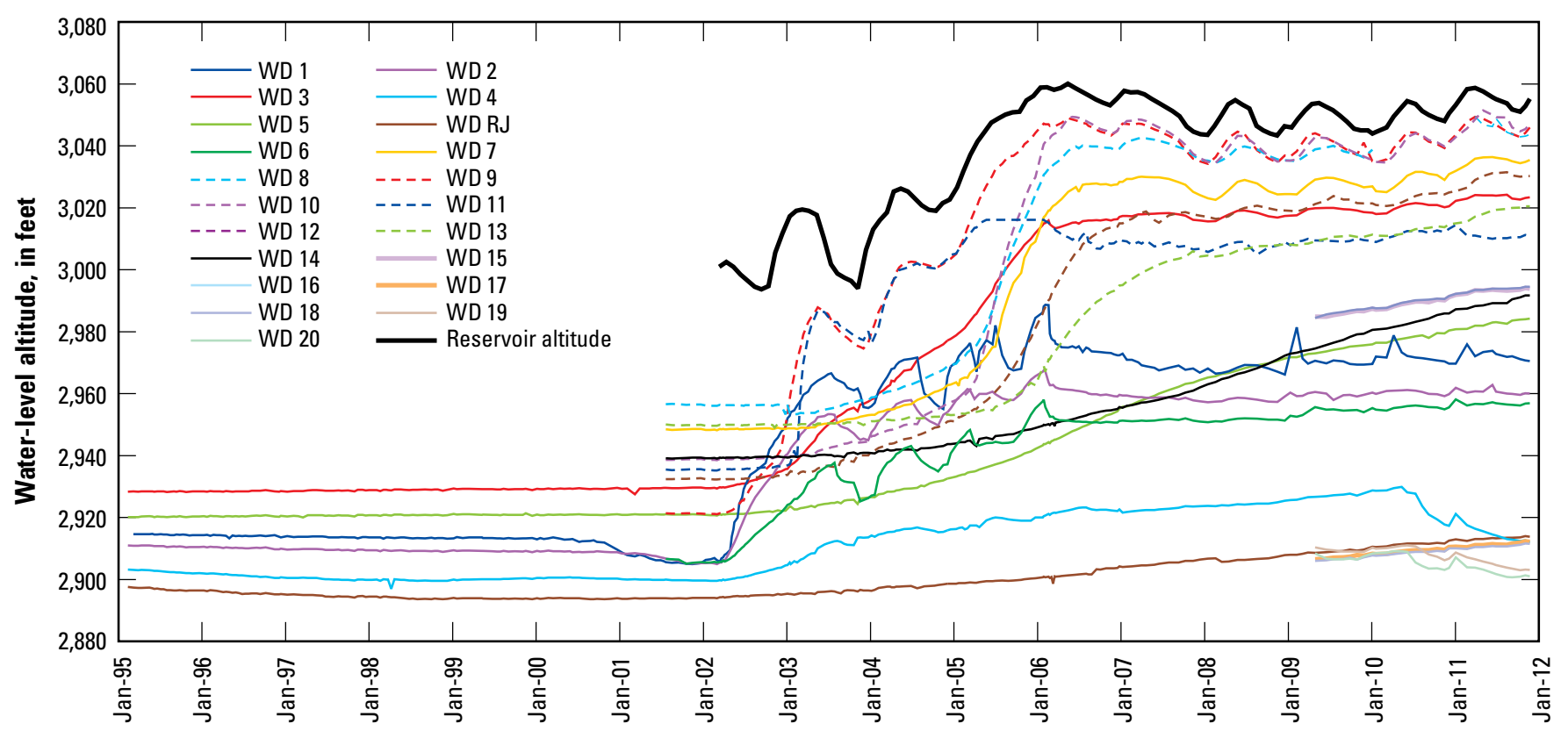

Figure 5. Water-level altitude in selected wells and Sand Hollow Reservoir, Sand Hollow, Utah, 1995-2011. 
Yearly water-level measurements from a long-term monitoring well (C-42-14)12dbb-1, located 1.4 mi north of Sand Hollow Reservoir, show water levels returning to altitudes observed in the mid-1970s (Burden and others, 2012) by 2011.

$B$ ased on water-level measurements in 21 monitoring wells, altitudes of the groundwater near Sand H ollow Reservoir during J anuary 2012 ranged from 2,901 to 3,051 ft (fig. 6). The reservoir altitude during this same period was about $3,058 \mathrm{ft}$. The lines on figure 6 show the estimated potentiometric contours in the aquifer (lines of equal groundwaterlevel altitude), and the arrows indicate the generalized direction of horizontal groundwater flow away from the reservoir. Horizontal hydraulic gradients, calculated by dividing the difference in water-level altitudes between two points by the distance separating these locations, indicate the potential horizontal direction of groundwater flow. The steepest horizontal hydraulic gradients are beneath the N orth and West Dams and generally decline with increasing distance from the reservoir. The steep gradients beneath the dams are the combined result of low-conductivity materials at the core of the dams and pumping in wells and drains at the base of both dams. For example, the horizontal hydraulic gradient between Sand Hollow Reservoir (reservoir altitude of 3,058 ft) and WD 1 (groundwater altitude of 2,971 ft) in 2011 was $0.132 \mathrm{ft} / \mathrm{ft}$, whereas the gradient between WD 6 (groundwater altitude of 2,957 ft) and W D $19(2,903 \mathrm{ft})$ was $0.013 \mathrm{ft} / \mathrm{ft}$. In 2011, the broader regional gradient between W D 9 (3,046 ft altitude) and W D RJ (2,914 ft altitude) was $0.021 \mathrm{ft} / \mathrm{ft}$. In comparison, the hydraulic gradient between these same tw o wells in 2004 was $0.017 \mathrm{ft} / \mathrm{ft}$ (fig. 7 of Heilweil and others, 2005). In general, groundwater, moves laterally away from the reservoir in all directions (fig. 6). The flow paths shown to the south, east, and west of the reservoir, however, likely curve around toward the north farther away from the reservoir. A syncline with an axis and plunge oriented in a northeasterly direction underlies Sand Hollow and controls the direction of all natural groundwater discharge from Sand Hollow. This natural discharge is primarily as seepage to the Virgin River to the north (fig. 1; Heilweil and others, 2000). In addition, the erosional extent of the Navajo Sandstone to the south and west of Sand Hollow, and the Hurricane Fault to the east, act as flow boundaries for the system.

\section{Surface-Water Inflow to and Outflow from Sand Hollow Reservoir}

Surface water is pumped into and flows out of Sand Hollow Reservoir through a 60-in diameter pipeline that enters through an inlet structure at the North Dam (fig. 2). This pipeline is part of a network of pipelines that connect the Virgin River, Sand Hollow Reservoir, Quail Creek Reservoir, and the Quail Creek R eservoir Water Treatment Facility. The W CW CD has the capability to move water within this network of pipelines by using gravity-induced flow and inline pumping. Sand Hollow Reservoir is currently managed to maximize groundwater recharge and little surface water has been removed from the reservoir. Monthly surface-water inflow to and outflow from Sand Hollow Reservoir is shown in table 1. The "Monthly pump station inflow or outflow" column in this table is the amount of Sand Hollow Reservoir surface water coming in from the Virgin River or going out to $Q$ uail Creek Reservoir, the Q uail Creek Water Treatment Plant, or other facilities (fig. 1). These data were collected at the WCWCD pump station located about 1 mi north of the North Dam. Five pumps, each with Sparling Tigermag totalizing flow meters, are linked to a computer system that combines and records total daily discharge in gallons. The flow meters have electronic modules on which calibration diagnostics are performed monthly by the WCW CD. Each module is removed annually for factory recalibration.

A wetter period during 2004 and 2005 resulted in above average snow pack in the Virgin River watershed, allowing the WCWCD to divert larger amounts of surface water to Sand Hollow Reservoir from the Virgin River and fill the reservoir to nearly full storage capacity by February 2006 . Because 2006 was a dry year (only 2.1 in of total rainfall recorded at Sand Hollow compared to the annual average of 7.0 in from 1998 through 2011), very little water was diverted from the Virgin River to the reservoir. Larger amounts of precipitation during the latter half of 2007 through early 2011 allowed for increased diversions and storage in the reservoir.

The "Monthly drain and spring return flow to reservoir" column in table 1 is the portion of discharge to the three drains that is pumped back into Sand Hollow Reservoir. The "Monthly outflow to Sand Hollow Resort" column is the amount of water required by the resort that cannot be met by discharge to the North Dam drain and is fulfilled by outflow of stored water from Sand Hollow Reservoir. Therefore, the "Monthly total inflow or outflow to/from reservoir" column is a sum of the pump station inflow/outflow, the drain and spring return flow, and the outflow to Sand Hollow Resort (table 1).

The "Monthly pump station inflow or outflow" column is unchanged from Heilweil and M arston (2011) and is comparable to the "Total surface-water inflow or outflow" column in table 7 of Heilweil and others (2005) from M arch 2002 to August 2004, the "Monthly surface-water inflow or outflow" column in table 2 of Heilweil and Susong (2007) from September 2004 to A ugust 2006, and the "M onthly net surfacewater inflow/outflow" column in table 2 of Heilweil and others (2009) from September 2006 to December 2007.

A s in Heilweil and M arston (2011), both "M onthly drain and spring return flow to reservoir" and "Monthly outflow to Sand Hollow Resort" are included in calculations of total inflow to and outflow from the reservoir. These amounts are added to the "Monthly pump station inflow or outflow" and summed in the "Monthly total inflow or outflow to/from reservoir" column. Monthly total inflow/outflow amounts from $M$ arch 2002 through December 2011 ranged from about $-5,000$ acre-ft to 6,600 acre-ft. Approximately 199,000 acre-ft of total net inflow were pumped into Sand Hollow Reservoir from the Virgin River from 2002 through 2011. 


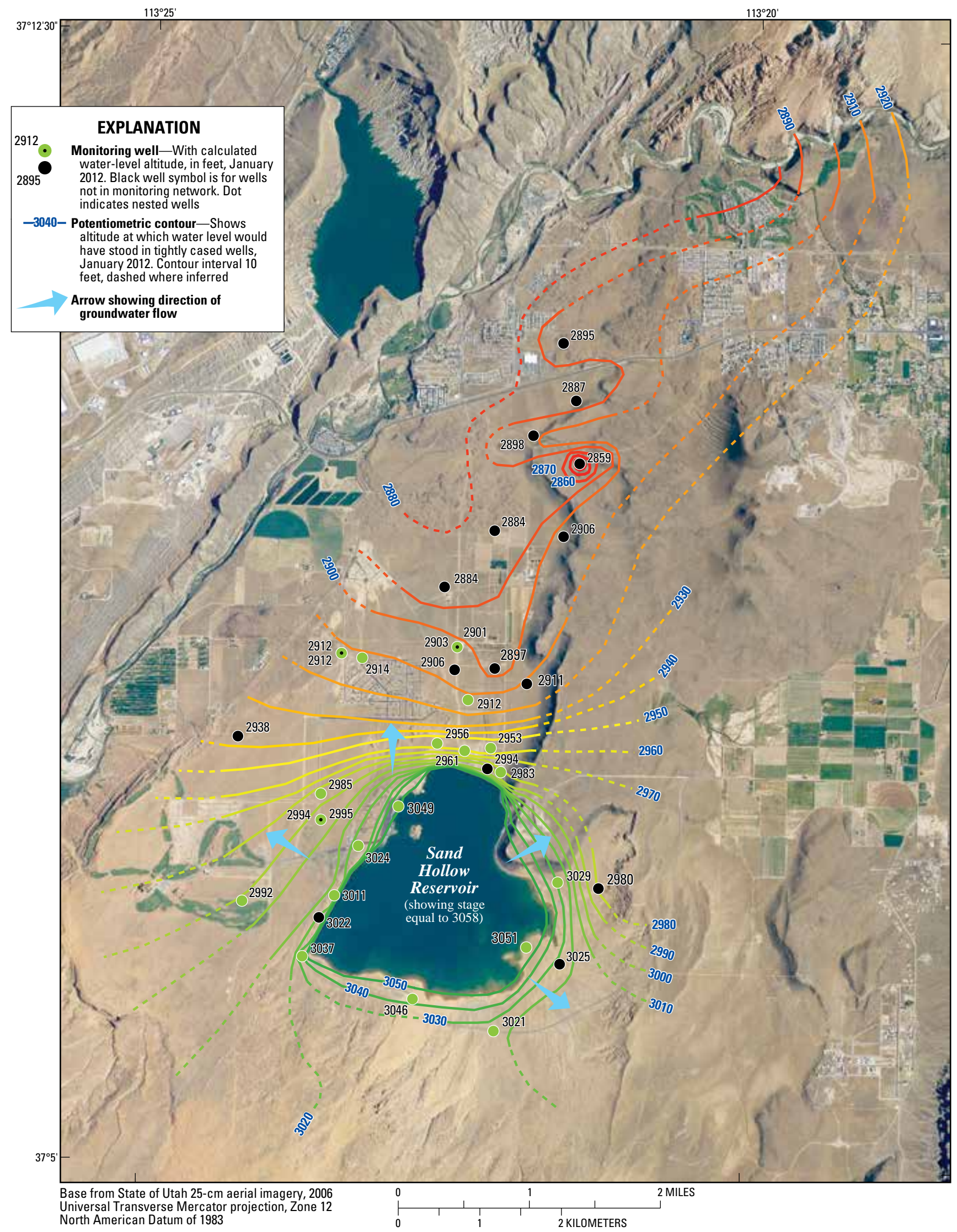

Figure 6. Potentiometric surface of the Navajo aquifer in January 2012, Sand Hollow, Utah. 


\section{Meteorological Data}

M eteorological data have been collected at the WCWCD weather station (fig. 2) in Sand Hollow since January 1998. B eginning in 2010, data from a Soil Climate A nalysis N etwork (SCA N) Sand Hollow weather station operated by the $\mathrm{N}$ atural Resources Conservation Service (NRCS) replaced the collection of data by the WCWCD weather station. The NRCS Sand Hollow SCAN station is located south of the reservoir within Sand Hollow basin in proximity to the WCWCD station (fig. 2). Data from both weather stations have been used for evaluating evaporation and precipitation, which are required for calculating monthly recharge from Sand Hollow Reservoir. Parameters measured include air temperature, wind speed, wind direction, precipitation, relative humidity, and incoming solar radiation. Instrumentation includes a temperature and relative humidity probe, a wind direction and speed monitor, a tipping bucket rain gage, and a solar radiometer. Sensors collect data every minute, and average hourly and daily values are computed and stored on a data logger (with the exception of precipitation, which is summed rather than averaged). The solar radiation and temperature data were used for calculating evaporation (using the version of the J enson and Haise method found in M cGuinness and B ordne, 1971; see following). The other data were collected to permit calculations of evaporation using other methods.
From J anuary 13,1998 , to D ecember 30,2011 , daily average air temperature ranged from -5 to $37^{\circ} \mathrm{C}$. The coldest temperatures during the year typically were in December and January, when minimum air temperatures occasionally were bel ow $-8^{\circ} \mathrm{C}$. The warmest temperatures were typically in July, when maximum air temperatures occasionally approached $45^{\circ} \mathrm{C}$. Daily average solar radiation ranged from 34 to 840 calories per square centimeter per day. The minimum daily averages are typically in December and January, and the maximum daily averages are typically in June and J uly.

$\mathrm{M}$ onthly precipitation has been recorded at Sand $\mathrm{Hol}$ low w eather station continuously from J anuary 1998 through December 2011, except for two periods when malfunctioning instrumentation resulted in data loss: December 26, 2008 to J anuary 3, 2009, and September 28 to November 16, 2009. Precipitation amounts during these two periods were estimated on the basis of data from the nearby St. George Southgate Golf Course weather station (\#427516; http://www.wrcc.dri. edu/cgi-bin/cliMAI N.pl?ut7516). F rom J anuary 1998 through December 2011, monthly precipitation ranged from 0 to about 4.3 in (fig. 7) and averaged about 0.6 in. Average annual precipitation during the 14-year period from 1998 through 2011 was $7.0 \mathrm{in}$. A nnual precipitation was about $10.2 \mathrm{in}, 10.6 \mathrm{in}$, and 15.7 in during 2004, 2005, and 2010, respectively, indicative of wetter-than-normal conditions at Sand Hollow.

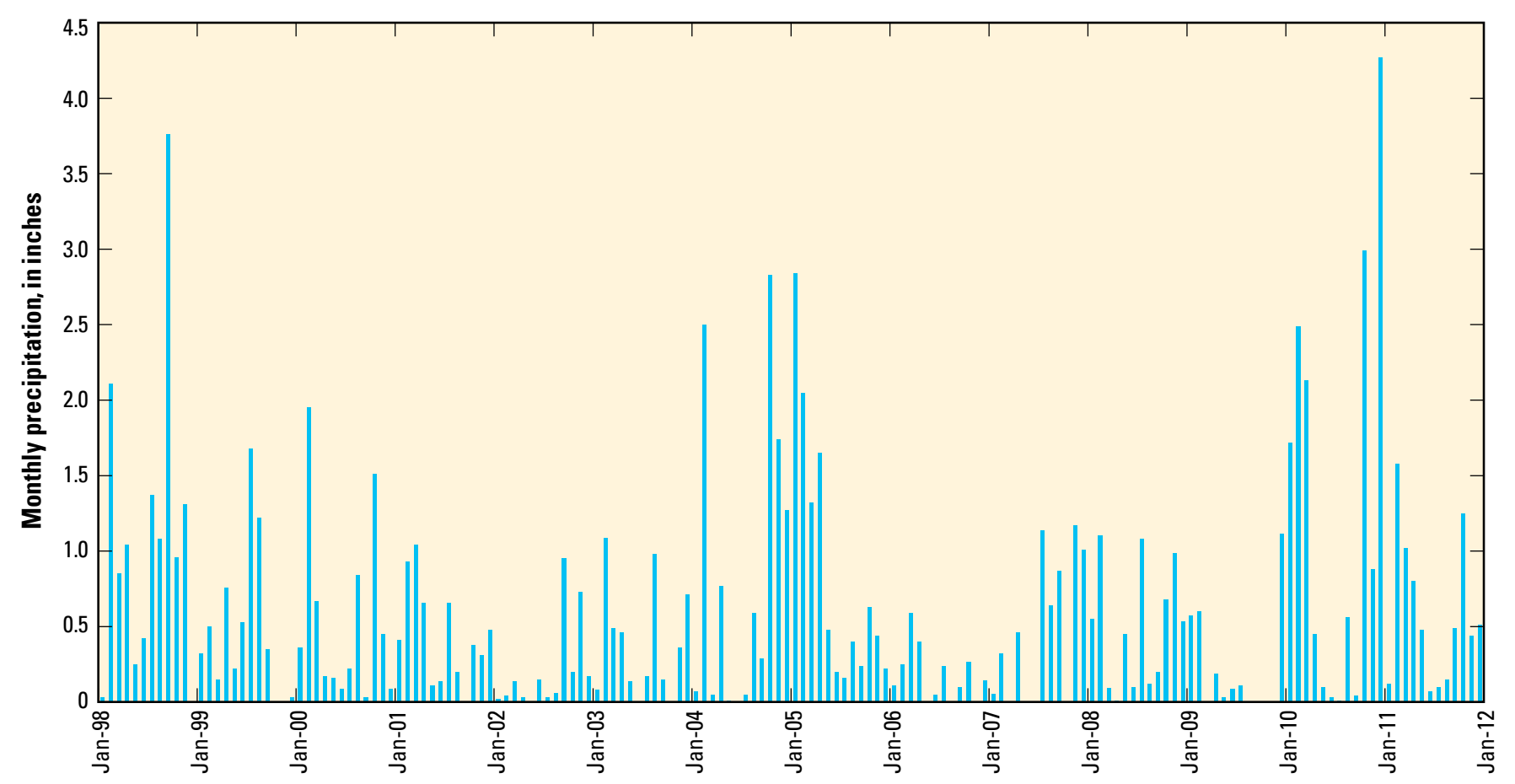

Figure 7. Monthly precipitation at Sand Hollow, Utah, 1998-2011. 
Table 1. Reservoir data, evaporation, and calculated recharge from Sand Hollow Reservoir, Utah, 2002-11.

[Reservoir altitude and Reservoir storage: value is from the last day of each month; Reservoir surface area: value is an average of the daily values for each month; 20,2 sigma; - , no data available]

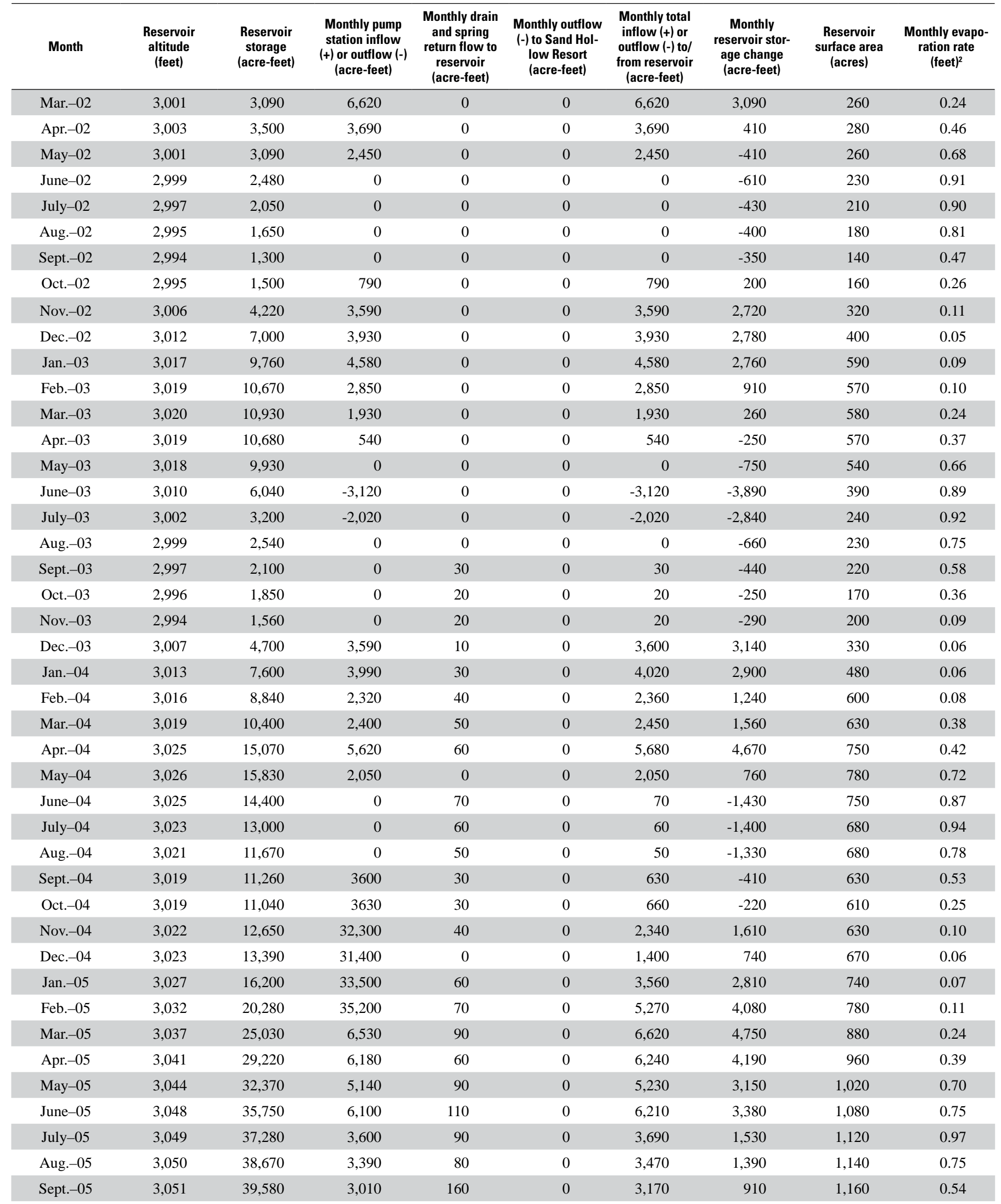


Table 1. Reservoir data, evaporation, and calculated recharge from Sand Hollow Reservoir, Utah, 2002-11.

[Reservoir altitude and Reservoir storage: value is from the last day of each month; Reservoir surface area: value is an average of the daily values for each month; $2 o, 2$ sigma; - , no data available]

\begin{tabular}{|c|c|c|c|c|c|c|c|}
\hline Month & $\begin{array}{l}\text { Monthly } \\
\text { evaporation } \\
\text { (acre-feet) }\end{array}$ & $\begin{array}{l}\text { Monthly precip- } \\
\text { itation } \\
\text { (acre-feet) }\end{array}$ & $\begin{array}{l}\text { Monthly ground- } \\
\text { water recharge } \\
\text { (acre-feet) }\end{array}$ & $\begin{array}{l}\text { Monthly ground- } \\
\text { water recharge } \\
\text { uncertainty, } 2 \sigma \\
\text { (percent) }\end{array}$ & $\begin{array}{c}\text { Monthly ground- } \\
\text { water recharge } \\
\text { uncertainty, } 2 \sigma \\
\text { (acre-feet) }\end{array}$ & $\begin{array}{l}\text { Groundwater } \\
\text { recharge rate } \\
\text { (feet/day) }\end{array}$ & $\begin{array}{c}\text { Average bottom } \\
\text { water temperature } \\
\text { (degrees Celsius) }\end{array}$ \\
\hline M ar.-02 & 60 & - & 3,470 & 6.7 & 232 & 0.430 & 10 \\
\hline A pr.-02 & 130 & - & 3,150 & 5.9 & 187 & 0.383 & 16 \\
\hline M ay-02 & 180 & - & 2,680 & 6.6 & 176 & 0.330 & 22 \\
\hline June-02 & 210 & - & 400 & 12.6 & 50 & 0.058 & 22 \\
\hline July-02 & 190 & - & 240 & 13.1 & 31 & 0.040 & 23 \\
\hline A ug.-02 & 150 & - & 250 & 12.7 & 32 & 0.044 & 24 \\
\hline Sept.-02 & 70 & - & 280 & 11.7 & 33 & 0.070 & 21 \\
\hline Oct.-02 & 40 & - & 550 & 6.6 & 36 & 0.110 & 15 \\
\hline Nov.-02 & 30 & - & 840 & 7.2 & 61 & 0.090 & 9 \\
\hline Dec.-02 & 20 & - & 1,130 & 7.1 & 80 & 0.090 & 6 \\
\hline Jan.-03 & 50 & - & 1,770 & 7.0 & 123 & 0.097 & 5 \\
\hline Feb.-03 & 60 & - & 1,880 & 6.4 & 121 & 0.118 & 7 \\
\hline M ar.-03 & 140 & - & 1,530 & 6.5 & 99 & 0.085 & 10 \\
\hline A pr.-03 & 210 & - & 580 & 9.4 & 55 & 0.034 & 16 \\
\hline M ay-03 & 360 & - & 390 & 13.2 & 52 & 0.023 & 22 \\
\hline June-03 & 350 & - & 420 & 8.4 & 35 & 0.036 & 22 \\
\hline July-03 & 220 & - & 600 & 8.4 & 51 & 0.081 & 23 \\
\hline Aug.-03 & 170 & - & 490 & 12.0 & 59 & 0.069 & 24 \\
\hline Sept.-03 & 130 & - & 340 & 12.3 & 42 & 0.052 & 21 \\
\hline Oct.-03 & 60 & - & 210 & 11.9 & 25 & 0.040 & 15 \\
\hline Nov.-03 & 20 & - & 290 & 10.6 & 31 & 0.048 & 9 \\
\hline Dec.-03 & 20 & - & 440 & 7.4 & 32 & 0.043 & 6 \\
\hline Jan.-04 & 30 & - & 1,090 & 7.2 & 78 & 0.073 & 5 \\
\hline Feb.-04 & 40 & - & 1,080 & 6.9 & 74 & 0.064 & 6 \\
\hline M ar.-04 & 240 & - & 650 & 7.7 & 50 & 0.033 & 11 \\
\hline A pr.-04 & 310 & - & 700 & 7.6 & 53 & 0.031 & 15 \\
\hline M ay- 04 & 560 & - & 730 & 8.6 & 63 & 0.030 & 18 \\
\hline J une- 04 & 650 & - & 850 & 13.1 & 112 & 0.038 & 21 \\
\hline July-04 & 640 & - & 820 & 13.1 & 108 & 0.039 & 23 \\
\hline A ug.-04 & 530 & - & 850 & 12.8 & 109 & 0.040 & 24 \\
\hline Sept.-04 & 330 & - & 710 & 10.2 & 73 & 0.038 & 22 \\
\hline Oct.-04 & 150 & - & 730 & 8.4 & 61 & 0.039 & 18 \\
\hline Nov.-04 & 70 & - & 660 & 7.3 & 48 & 0.035 & 12 \\
\hline Dec.-04 & 40 & - & 620 & 7.0 & 43 & 0.030 & 7 \\
\hline Jan.-05 & 50 & - & 700 & 7.3 & 51 & 0.031 & 6 \\
\hline Feb.-05 & 80 & 130 & 1,240 & 7.3 & 91 & 0.057 & 8 \\
\hline M ar. -05 & 210 & 100 & 1,760 & 7.4 & 130 & 0.065 & 11 \\
\hline A pr.-05 & 370 & 130 & 1,810 & 7.5 & 136 & 0.063 & 13 \\
\hline M ay-05 & 710 & 40 & 1,410 & 7.9 & 112 & 0.045 & 15 \\
\hline June-05 & 810 & 20 & 2,040 & 7.8 & 160 & 0.063 & 19 \\
\hline July-05 & 1,080 & 10 & 1,090 & 8.8 & 96 & 0.031 & 22 \\
\hline A ug.-05 & 850 & 40 & 1,270 & 8.5 & 108 & 0.036 & 22 \\
\hline Sept.-05 & 630 & 20 & 1,650 & 8.1 & 133 & 0.047 & 23 \\
\hline
\end{tabular}




\section{Assessment of Managed Aquifer Recharge at Sand Hollow Reservoir, Updated to Conditions in 2012}

Table 1. Reservoir data, evaporation, and calculated recharge from Sand Hollow Reservoir, Utah, 2002-11.-Continued

[Reservoir altitude and Reservoir storage: value is from the last day of each month; Reservoir surface area: value is an average of the daily values for each month; 20,2 sigma; - , no data available]

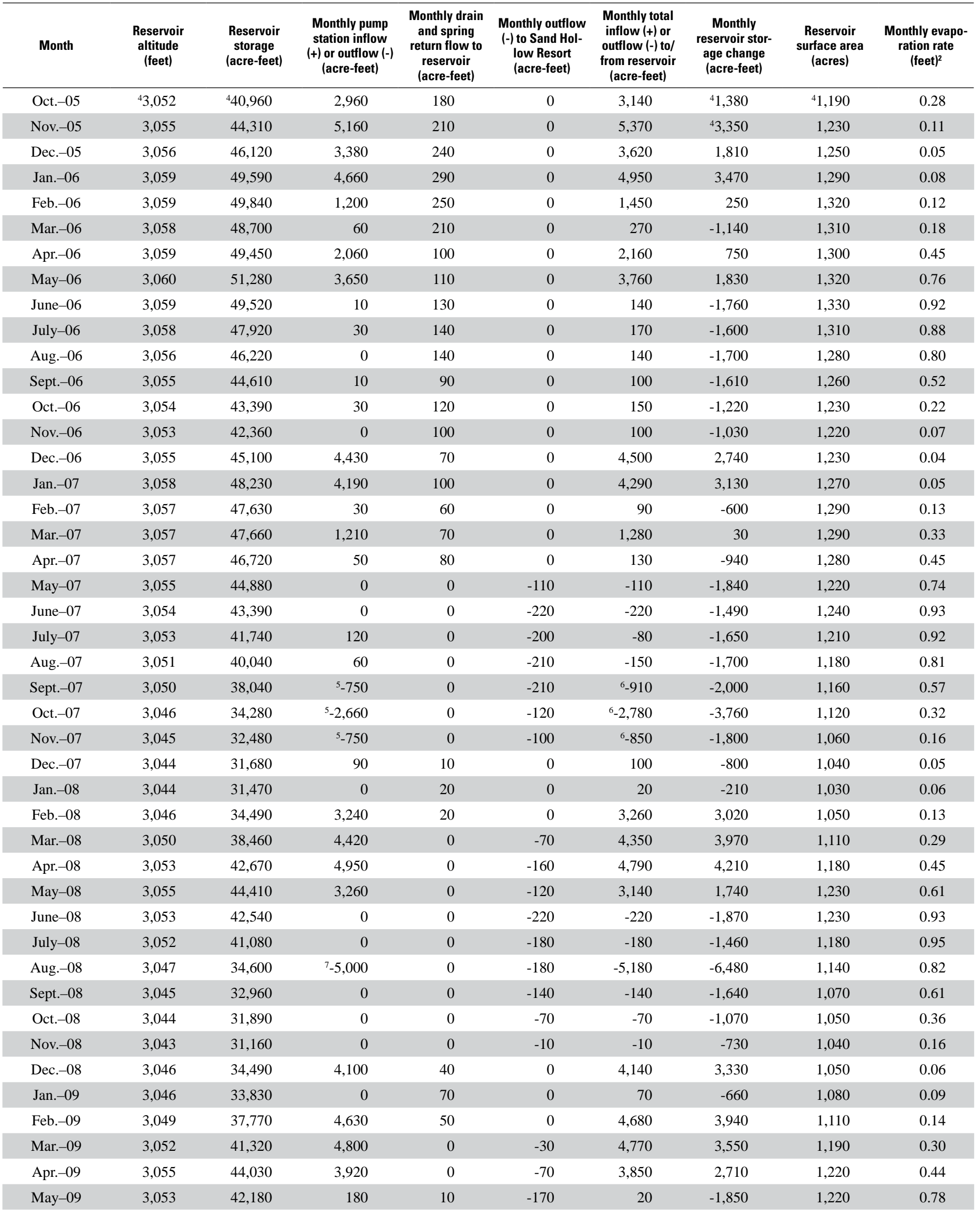


Table 1. Reservoir data, evaporation, and calculated recharge from Sand Hollow Reservoir, Utah, 2002-11.-Continued [Reservoir altitude and Reservoir storage: value is from the last day of each month; Reservoir surface area: value is an average of the daily values for each month; $2 \sigma, 2$ sigma; - - no data available]

\begin{tabular}{|c|c|c|c|c|c|c|c|}
\hline Month & $\begin{array}{l}\text { Monthly } \\
\text { evaporation } \\
\text { (acre-feet) }\end{array}$ & $\begin{array}{l}\text { Monthly precip- } \\
\text { itation } \\
\text { (acre-feet) }\end{array}$ & $\begin{array}{l}\text { Monthly ground- } \\
\text { water recharge } \\
\text { (acre-feet) }\end{array}$ & $\begin{array}{l}\text { Monthly ground- } \\
\text { water recharge } \\
\text { uncertainty, } 2 \sigma \\
\text { (percent) }\end{array}$ & $\begin{array}{l}\text { Monthly ground- } \\
\text { water recharge } \\
\text { uncertainty, } 2 \sigma \\
\text { (acre-feet) }\end{array}$ & $\begin{array}{l}\text { Groundwater } \\
\text { recharge rate } \\
\text { (feet/day) }\end{array}$ & $\begin{array}{c}\text { Average bottom } \\
\text { water temperature } \\
\text { (degrees Celsius) }\end{array}$ \\
\hline Oct.-05 & ${ }^{4} 330$ & 60 & 1,490 & 7.6 & 113 & 0.040 & 18 \\
\hline Nov. -05 & 140 & 40 & 1,920 & 7.2 & 138 & 0.052 & 15 \\
\hline Dec.-05 & 60 & 20 & 1,770 & 6.9 & 122 & 0.046 & 8 \\
\hline Jan.-06 & 100 & 10 & 1,390 & 7.3 & 101 & 0.035 & 8 \\
\hline Feb.-06 & 160 & 30 & 1,070 & 7.3 & 78 & 0.029 & 7 \\
\hline M ar.-06 & 240 & 60 & 1,230 & 11.4 & 140 & 0.030 & 9 \\
\hline A pr.-06 & 580 & 40 & 870 & 8.7 & 76 & 0.022 & 12 \\
\hline M ay-06 & 1,000 & 0 & 930 & 8.7 & 81 & 0.023 & 17 \\
\hline J une-06 & 1,220 & 10 & 690 & 14.1 & 97 & 0.017 & 21 \\
\hline July-06 & 1,160 & 30 & 640 & 14.1 & 90 & 0.016 & 25 \\
\hline A ug.-06 & 1,020 & 0 & 820 & 13.8 & 113 & 0.021 & 27 \\
\hline Sept.-06 & 650 & 10 & 1,070 & 12.8 & 137 & 0.028 & 24 \\
\hline Oct.-06 & 270 & 30 & 1,130 & 11.6 & 132 & 0.030 & 19 \\
\hline Nov.-06 & 90 & 0 & 1,040 & 10.8 & 112 & 0.028 & 14 \\
\hline Dec.-06 & 60 & 10 & 1,710 & 7.0 & 120 & 0.045 & 8 \\
\hline Jan.-07 & 60 & 10 & 1,110 & 7.2 & 80 & 0.028 & 4 \\
\hline Feb.-07 & 170 & 30 & 550 & 11.9 & 65 & 0.015 & 4 \\
\hline M ar.- 07 & 430 & 0 & 820 & 9.0 & 73 & 0.021 & 8 \\
\hline A pr.-07 & 580 & 50 & 540 & 13.4 & 73 & 0.014 & 12 \\
\hline M ay-07 & 900 & 0 & 830 & 13.3 & 110 & 0.022 & 15 \\
\hline J une- 07 & 1,150 & 0 & 120 & 14.4 & 17 & 0.003 & 19 \\
\hline July-07 & 1,110 & 110 & 560 & 13.5 & 76 & 0.015 & 22 \\
\hline A ug.-07 & 960 & 60 & 650 & 13.3 & 87 & 0.018 & 26 \\
\hline Sept.-07 & 660 & 80 & 510 & 10.8 & 55 & 0.015 & 25 \\
\hline Oct.-07 & 360 & 0 & 620 & 8.6 & 53 & 0.018 & 18 \\
\hline Nov.- 07 & 170 & 100 & 880 & 9.3 & 82 & 0.028 & 14 \\
\hline Dec.-07 & 50 & 90 & 940 & 10.0 & 94 & 0.029 & 8 \\
\hline Jan.-08 & 60 & 50 & 220 & 11.9 & 26 & 0.007 & 4 \\
\hline Feb.-08 & 140 & 100 & 200 & 7.7 & 15 & 0.007 & 4 \\
\hline M ar.-08 & 320 & 10 & 70 & 7.8 & 5 & 0.002 & 8 \\
\hline A pr.-08 & 530 & 0 & 50 & 8.0 & 4 & 0.001 & 11 \\
\hline M ay-08 & 750 & 50 & 700 & 8.5 & 59 & 0.018 & 15 \\
\hline June-08 & 1,140 & 10 & 520 & 13.8 & 72 & 0.014 & 19 \\
\hline July-08 & 1,120 & 110 & 270 & 14.2 & 38 & 0.007 & 22 \\
\hline A ug. -08 & 940 & 10 & 370 & 8.7 & 32 & 0.010 & 17 \\
\hline Sept.-08 & 650 & 20 & 870 & 12.8 & 111 & 0.027 & 19 \\
\hline Oct.-08 & 370 & 60 & 690 & 12.5 & 86 & 0.021 & 19 \\
\hline Nov.-08 & 160 & 80 & 640 & 11.6 & 75 & 0.021 & 14 \\
\hline Dec.-08 & 60 & 50 & 800 & 7.4 & 59 & 0.025 & 9 \\
\hline Jan.-09 & 100 & 50 & 680 & 11.2 & 76 & 0.020 & 5 \\
\hline Feb.-09 & 150 & 60 & 650 & 7.5 & 49 & 0.021 & 7 \\
\hline M ar.-09 & 360 & 0 & 860 & 7.7 & 66 & 0.023 & 9 \\
\hline A pr.-09 & 530 & 20 & 630 & 8.0 & 50 & 0.017 & 11 \\
\hline M ay-09 & 950 & 0 & 920 & 12.9 & 119 & 0.024 & 13 \\
\hline
\end{tabular}


Table 1. Reservoir data, evaporation, and calculated recharge from Sand Hollow Reservoir, Utah, 2002-11.-Continued [Reservoir altitude and Reservoir storage: value is from the last day of each month; Reservoir surface area: value is an average of the daily values for each month; 20,2 sigma; - , no data available]

\begin{tabular}{|c|c|c|c|c|c|c|c|c|c|}
\hline Month & $\begin{array}{l}\text { Reservoir } \\
\text { altitude } \\
\text { (feet) }\end{array}$ & $\begin{array}{c}\text { Reservoir } \\
\text { storage } \\
\text { (acre-feet) }\end{array}$ & $\begin{array}{c}\text { Monthly pump } \\
\text { station inflow } \\
\text { (+) or outflow (-) } \\
\text { (acre-feet) }\end{array}$ & $\begin{array}{l}\text { Monthly drain } \\
\text { and spring } \\
\text { return flow to } \\
\text { reservoir } \\
\text { (acre-feet) }\end{array}$ & $\begin{array}{l}\text { Monthly outflow } \\
\text { (-) to Sand Hol- } \\
\text { low Resort } \\
\text { (acre-feet) }\end{array}$ & $\begin{array}{c}\text { Monthly total } \\
\text { inflow (+) or } \\
\text { outflow (-) to/ } \\
\text { from reservoir } \\
\text { (acre-feet) }\end{array}$ & $\begin{array}{l}\text { Monthly } \\
\text { reservoir stor- } \\
\text { age change } \\
\text { (acre-feet) }\end{array}$ & $\begin{array}{l}\text { Reservoir } \\
\text { surface area } \\
\text { (acres) }\end{array}$ & $\begin{array}{l}\text { Monthly evapo- } \\
\text { ration rate } \\
\quad \text { (feet })^{2}\end{array}$ \\
\hline July-09 & 3,050 & 38,700 & 220 & 0 & -170 & 50 & $-1,900$ & 1,170 & 0.96 \\
\hline A ug.-09 & 3,049 & 36,960 & 210 & 0 & -150 & 60 & $-1,740$ & 1,140 & 0.80 \\
\hline Nov.-09 & 3,045 & 32,960 & 180 & 10 & -20 & 170 & -980 & 1,070 & 0.16 \\
\hline Dec.-09 & 3,044 & 32,320 & 200 & 40 & 0 & 240 & -640 & 1,050 & 0.05 \\
\hline Jan.-10 & 3,044 & 31,890 & 0 & 50 & 0 & 50 & -430 & 1,040 & 0.07 \\
\hline Feb.- 10 & 3,044 & 31,470 & 0 & 40 & 0 & 40 & -420 & 1,040 & 0.10 \\
\hline June-10 & 3,054 & 43,660 & 3,890 & 80 & -190 & 3,780 & 1,850 & 1,220 & 0.81 \\
\hline July-10 & 3,053 & 42,300 & 570 & 100 & -240 & 430 & $-1,360$ & 1,220 & 0.91 \\
\hline A ug. -10 & 3,051 & 40,240 & 0 & 80 & -220 & -140 & $-2,060$ & 1,190 & 0.77 \\
\hline Sept.-10 & 3,050 & 38,350 & 0 & 80 & -210 & -130 & $-1,890$ & 1,160 & 0.60 \\
\hline Oct.-10 & 3,049 & 37,310 & 0 & 100 & -90 & 10 & $-1,040$ & 1,140 & 0.29 \\
\hline Nov.-10 & 3,048 & 36,620 & 0 & 90 & -40 & 50 & -690 & 1,120 & 0.13 \\
\hline Dec.-10 & 3,051 & 40,240 & 4,290 & 90 & -10 & 4,370 & 3,620 & 1,150 & 0.06 \\
\hline Jan.-11 & 3,054 & 43,960 & 5,650 & 60 & -10 & 5,700 & 3,720 & 1,200 & 0.09 \\
\hline Feb.-11 & 3,057 & 47,750 & 4,540 & 40 & -30 & 4,550 & 3,790 & 1,260 & 0.11 \\
\hline Sept.-11 & 3,052 & 40,600 & 0 & 20 & -150 & -130 & $-1,390$ & 1,190 & 0.61 \\
\hline Oct.-11 & 3,051 & 39,910 & 0 & 30 & -70 & -40 & -690 & 1,170 & 0.35 \\
\hline Nov.-11 & 3,054 & 43,490 & 3,980 & 30 & -30 & 3,980 & 3,580 & 1,200 & 0.15 \\
\hline Dec.-11 & 3,058 & 48,010 & 4,990 & 20 & -10 & 5,000 & 4,520 & 1,260 & 0.07 \\
\hline Total & - & - & - & - & - & 199,110 & - & - & - \\
\hline
\end{tabular}

${ }^{1}$ Negative (-) values indicate flows out of Sand Hollow to Quail Creek Water Treatment Plant or to Quail Creek Reservoir.

${ }^{2}$ M onthly evaporation rate from February 2007 through December 2009 was calculated with a correction factor to account for higher solar radiation measurements with new instrument.

${ }^{3}$ Because of problems with monitoring equipment, inflows from September 2004 through February 2005 are estimated based on previous inflow history and changes in reservoir altitude.

${ }^{4}$ Revised value based on refined reservoir altitude estimate for October 2005.

${ }^{5}$ Monthly pump station outflow was increased from amount reported in Heilweil and others (2009a) based on reservoir altitude relations.

${ }^{6}$ Monthly total outflow was increased from amount reported by Washington County Water Conservancy District based on reservoir altitude relations.

${ }^{7}$ Monthly pump station outflow was increased from previously reported amount (Heilweil and others, 2009a) based on reservoir altitude relations.

${ }^{8}$ Sand Hollow rain gauge not functioning; values of 0 based on lack of precipitation from St. George precipitation station \#427516. 
Table 1. Reservoir data, evaporation, and calculated recharge from Sand Hollow Reservoir, Utah, 2002-11.-Continued [Reservoir altitude and Reservoir storage: value is from the last day of each month; Reservoir surface area: value is an average of the daily values for each month; 20,2 sigma; - , no data available]

\begin{tabular}{|c|c|c|c|c|c|c|c|}
\hline Month & $\begin{array}{l}\text { Monthly } \\
\text { evaporation } \\
\text { (acre-feet) }\end{array}$ & $\begin{array}{l}\text { Monthly precip- } \\
\text { itation } \\
\text { (acre-feet) }\end{array}$ & $\begin{array}{l}\text { Monthly ground- } \\
\text { water recharge } \\
\text { (acre-feet) }\end{array}$ & $\begin{array}{l}\text { Monthly ground- } \\
\text { water recharge } \\
\text { uncertainty, } 2 \sigma \\
\text { (percent) }\end{array}$ & $\begin{array}{l}\text { Monthly ground- } \\
\text { water recharge } \\
\text { uncertainty, } 2 \sigma \\
\text { (acre-feet) }\end{array}$ & $\begin{array}{l}\text { Groundwater } \\
\text { recharge rate } \\
\text { (feet/day) }\end{array}$ & $\begin{array}{l}\text { Average bottom } \\
\text { water temperature } \\
\text { (degrees Celsius) }\end{array}$ \\
\hline June-09 & 870 & 10 & 800 & 12.9 & 103 & 0.022 & 14 \\
\hline July-09 & 1,120 & 10 & 840 & 13.1 & 110 & 0.023 & 15 \\
\hline A ug.-09 & 910 & 0 & 890 & 12.8 & 114 & 0.025 & 16 \\
\hline Sept.-09 & 650 & 0 & 980 & 12.3 & 120 & 0.029 & 19 \\
\hline Oct.-09 & 320 & ${ }^{8} 0$ & 1,320 & 11.1 & 147 & 0.039 & 18 \\
\hline Nov.-09 & 170 & ${ }^{8} 0$ & 1,050 & 10.6 & 111 & 0.033 & 12 \\
\hline Dec.-09 & 60 & 100 & 920 & 9.6 & 88 & 0.028 & 7 \\
\hline Jan.-10 & 80 & 150 & 550 & 11.2 & 62 & 0.017 & 6 \\
\hline Feb.-10 & 110 & 220 & 570 & 11.5 & 65 & 0.018 & 7 \\
\hline M ar.-10 & 250 & 190 & 1,060 & 7.6 & 80 & 0.032 & 9 \\
\hline A pr.-10 & 400 & 40 & 1,440 & 7.6 & 109 & 0.041 & 11 \\
\hline M ay-10 & 660 & 10 & 1,050 & 8.0 & 84 & 0.029 & 14 \\
\hline J une-10 & 990 & 0 & 940 & 8.6 & 81 & 0.025 & 16 \\
\hline July-10 & 1,100 & 0 & 690 & 12.7 & 88 & 0.018 & 17 \\
\hline A ug.-10 & 920 & 60 & 1,060 & 13.0 & 138 & 0.029 & 17 \\
\hline Sept.-10 & 690 & 0 & 1,070 & 12.7 & 136 & 0.030 & 18 \\
\hline Oct.-10 & 320 & 280 & 1,010 & 12.0 & 121 & 0.029 & 19 \\
\hline Nov. -10 & 150 & 80 & 670 & 11.6 & 78 & 0.019 & 14 \\
\hline Dec.-10 & 70 & 410 & 1,090 & 7.5 & 82 & 0.031 & 9 \\
\hline Jan.-11 & 100 & 10 & 1,890 & 7.1 & 135 & 0.051 & 7 \\
\hline Feb.-11 & 140 & 170 & 790 & 7.5 & 60 & 0.020 & 6 \\
\hline M ar.-11 & 340 & 110 & 1,020 & 7.7 & 79 & 0.025 & 8 \\
\hline A pr.-11 & 560 & 90 & 560 & 13.1 & 73 & 0.014 & 11 \\
\hline M ay-11 & 760 & 50 & 800 & 13.1 & 105 & 0.020 & 13 \\
\hline J une-11 & 1,130 & 10 & 550 & 13.9 & 76 & 0.014 & 14 \\
\hline July-11 & 1,110 & 10 & 680 & 13.6 & 92 & 0.018 & 15 \\
\hline A ug.-11 & 1,130 & 20 & 430 & 13.9 & 60 & 0.011 & 16 \\
\hline Sept.-11 & 720 & 50 & 590 & 13.3 & 79 & 0.016 & 17 \\
\hline Oct.-11 & 420 & 120 & 350 & 13.4 & 47 & 0.010 & 18 \\
\hline Nov.-11 & 180 & 40 & 260 & 7.7 & 20 & 0.007 & 13 \\
\hline Dec.-11 & 90 & 50 & 440 & 7.5 & 33 & 0.011 & 7 \\
\hline Total & 49,780 & - & 105,790 & - & - & - & - \\
\hline
\end{tabular}

${ }^{1}$ Negative (-) values indicate flows out of Sand Hollow to Quail Creek Water Treatment Plant or to Quail Creek Reservoir.

${ }^{2}$ M onthly evaporation rate from February 2007 through December 2009 was calculated with a correction factor to account for higher solar radiation measurements with new instrument.

${ }^{3}$ Because of problems with monitoring equipment, inflows from September 2004 through February 2005 are estimated based on previous inflow history and changes in reservoir altitude.

${ }^{4}$ Revised value based on refined reservoir altitude estimate for October 2005.

${ }^{5}$ Monthly pump station outflow was increased from amount reported in Heilweil and others (2009a) based on reservoir altitude relations.

${ }^{6}$ Monthly total outflow was increased from amount reported by Washington County Water Conservancy District based on reservoir altitude relations

${ }^{7}$ Monthly pump station outflow was increased from previously reported amount (Heilweil and others, 2009a) based on reservoir altitude relations.

${ }^{8}$ Sand Hollow rain gauge not functioning; values of 0 based on lack of precipitation from St. George precipitation station \#427516. 


\section{Reservoir Water Temperature}

Continuous water-temperature measurements were made in Sand Hollow Reservoir and used for evaluating effects of water viscosity changes on seepage rates beneath the reservoir. A string of five thermistors was installed in January 2003 in the deepest part of Sand Hollow Reservoir, about $300 \mathrm{ft}$ from the N orth Dam. These thermistors were attached to a floating buoy at depths of about $0.3 \mathrm{ft}(\mathrm{R} 1), 3 \mathrm{ft}(\mathrm{R} 2), 10 \mathrm{ft}$ (R3), $15 \mathrm{ft}$ (R4), and $30 \mathrm{ft}$ (R5). A sixth thermistor (R6) was added on M ay 6, 2008, at a depth of $50 \mathrm{ft}$ (or at the bottom of the reservoir, if shallower). The thermistors $\left(\mathrm{Onset}^{\circledR} \mathrm{Hobo}^{\circledR}\right.$ TidbiT Water Temperature Data Loggers) are reported to have an accuracy of better than $0.5^{\circ} \mathrm{C}$ over the temperature range of 0 to 35ㄷ. Water temperature from J anuary 2003 through December 2011 ranged from about 1 to $30^{\circ} \mathrm{C}$. B oth the previous (J anuary 2003 through December 2007) and current (J anuary 2008 through December 2011) temperature data are shown in figure 8 . Water temperature data at the following locations were not recorded for periods exceeding 30 days because of problems with the thermistors, buoy, or both: $\mathrm{R} 1$ at the $0.3-\mathrm{ft}$ depth, January 1 to J une 4, 2008 and $M$ arch 31 to A ugust 4, 2010; R2 at the 3-ft depth, M arch 11 to J une 5, 2008; R 3 at the 10 -ft depth, M arch 11 to J uly 29, 2008; R 4 at the 15 -ft depth,
$M$ arch 11 to $M$ ay 5, 2008, A ugust 27 to 0 ctober 3, 2009, and September 8 to December 31, 2011; R5 at the 30-ft depth, J une 5 to J uly 29, 2008; and R6 at the 50-ft depth, J une 5 to July 29, 2008.

\section{Estimates of Managed Aquifer Recharge from Sand Hollow Reservoir}

Substantial amounts of surface water from Sand $\mathrm{Hol}$ low Reservoir infiltrate through the underlying sediments to recharge the Navajo Sandstone aquifer. This recharge either is captured by production wells for municipal supply, or it moves northward through the aquifer towards the Virgin River. Through 2011, withdrawals from production wells operated by the WCW CD at Sand Hollow have been permitted for the capture of natural recharge in Sand Hollow. These withdrawals are governed by different water rights than those associated with managed aquifer recharge from Sand Hollow Reservoir; withdrawal rights for this artificial recharge have not yet been exercised.

Recharge from Sand Hollow Reservoir is calculated as the residual with the following water-budget equation (modified from Heilweil and others, 2005):

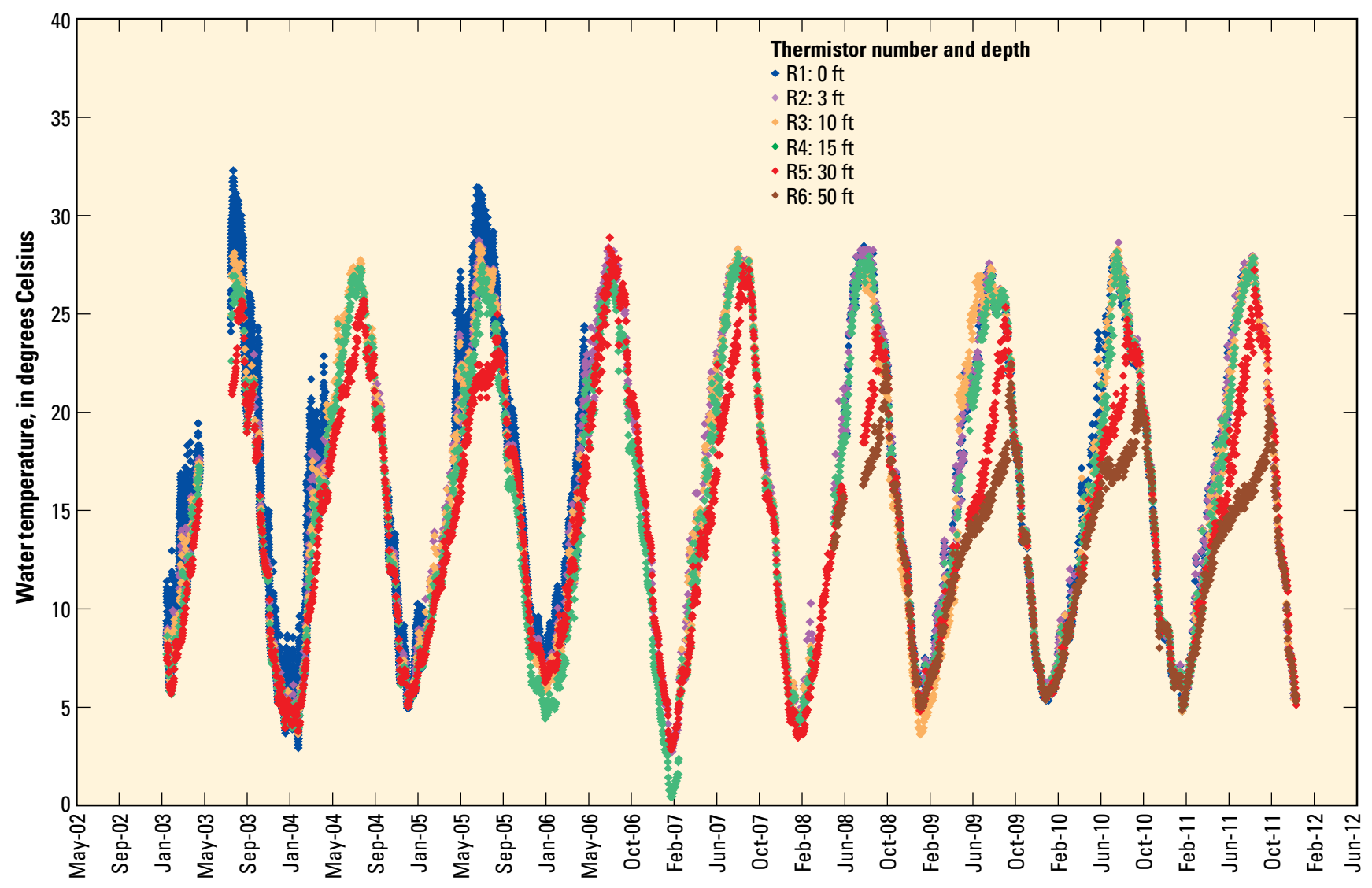

Figure 8. Daily water temperature at various depths in Sand Hollow Reservoir, Utah, 2003-11. 


$$
\mathrm{R}=\mathrm{I}_{\mathrm{sw}}+\mathrm{I}_{\mathrm{dr}}-\mathrm{O}_{\mathrm{sw}}+\mathrm{P} \pm \Delta S-\mathrm{E}
$$

where

$R$ is recharge,

$I_{\text {Sw }}$ is surface-water inflow,

$I_{d r}$ is drain return flow,

$\mathrm{O}_{\mathrm{SW}}$ is surface-water outflow,

$\mathrm{P}$ is the amount of precipitation falling directly on the reservoir,

$\Delta S$ is change in surface-water storage, and

$\mathrm{E}$ is evaporation.

All the variables in equation 1 are in units of acre-ft.

The following equation was developed to evaluate the uncertainty for each monthly recharge estimate:

$$
\mathrm{CU}=\Sigma\left[\left(\left|\mathrm{C}_{\mathrm{i}}\right| / \Sigma \mid C_{\mathrm{i}}\right) * \mathrm{U}_{\mathrm{i}}\right]
$$

where

$\mathrm{CU}$ is the composite uncertainty fraction (two standard deviation, $2 \sigma$ )

$\left|C_{i}\right|$ is the absolute value of each component of the water budget (acre-ft),

$\Sigma\left|C_{\mathrm{i}}\right| \quad$ is the sum of absolute values of all the waterbudget components (acre-ft), and

$U_{i}$ is the uncertainty fraction $(2 \sigma)$ for each individual water-budget component.

The small lest estimated uncertainty fraction is 0.05 (5 percent) for $I_{s w^{\prime}} I_{D^{\prime}}$ and $\mathrm{O}_{s w}$ because these flows are recorded using calibrated inline flow meters. The estimated uncertainty fraction for $\mathrm{P}$ is higher, at 0.10 (10 percent), because it is an indirect measurement made on the basis of nearby meteorological station data. Similarly, the estimated uncertainty fraction is also 0.10 (10 percent) for $\Delta S$ because changes in surface-water storage are based only on approximate reservoir water-level altitude/volume relations rather than direct measurements. The largest estimated uncertainty fraction is 0.20 (20 percent) for $E$, which is based on differences between alternative methods for estimating evaporation both at Sand Hollow and other areas (H eilweil and others, 2007; Rosenberry and others, 2007).

The first two reports documenting monthly groundwater recharge beneath Sand Hollow Reservoir through A ugust 2006 (Heilweil and others, 2005; Heilweil and Susong, 2007) did not include precipitation falling directly on the reservoir. B eginning with the third report (H eil weil and others, 2009a), and continuing in this report, an additional term for precipitation falling directly on the reservoir $(\mathrm{P})$ was included in equation 1 . The monthly amount of precipitation falling on the reservoir is calculated by multiplying the total monthly precipitation recorded by the Sand Hollow weather station by the average reservoir surface area for that month (based on reservoir water-level altitude to area relations for the reservoir; RB G Engineering, written commun., 2002; Washington County Water Conservancy District, written commun., 2006). The precipitation term in equation 1 , however, does not account for precipitation runoff to the reservoir. B ecause of high evaporation rates and permeable surficial soils, precipitation events seldom produce runoff that reaches the lower part of Sand Hollow (L. J essop, Washington County Water Conservancy District, oral commun., 2001), where the reservoir is situated.

M onthly water-budget values for Sand Hollow Reservoir are given in table 1 . Values are generally monthly averages or totals, except for reservoir altitude and storage, which are shown for the last day of each month. Values for "M onthly evaporation rate," "Monthly evaporation," and "Monthly groundwater recharge" from March 2002 through January 2005 and from January 2008 through December 2011 are monthly averages; during February 2005 through December 2007 , however, the values are the sum of daily measurements. Summing of daily evaporation estimates was discontinued after 2007 because comparison of daily and average monthly calculations during 2008 and 2009 showed little difference, and the equation used for calculating evapotranspiration is more appropriate for calculating average evaporation over longer periods (M CG uinness and B ordne, 1971).

\section{Changes in Reservoir Storage}

Changes in reservoir storage were calculated from daily reservoir water-level altitudes reported by the WCW CD by using al titude to volume relations (RBG Engineering, written commun., 2002). Since inception of the reservoir in 2002, surface-water storage increased to a maximum of about 51,000 acre- $\mathrm{ft}$ in M ay of 2006. From the latter half of 2006 through 2007, surface-water storage decreased to about 32,000 acre-ft, and during 2008 through 2010, surface-water storage varied between about 31,000 and 44,000 acre-ft. Following the abnormally wet winter and spring of 2010-11, surface-water storage was kept at a high level during 2011, varying between 40,000 to 50,000 acre-ft (table 1 ).

\section{Reservoir Evaporation}

The M cGuinness and B ordne (1971) version of the JensenHaise method was selected for calculating evaporation from Sand Hollow Reservoir during this study. A detailed comparison to results using other methods for estimating evaporation is given in Heilweil and others (2005). The M cGuinness and B ordne (1971) version of the J ensen-H aise method is based on the relation:

$$
P E T=\left\{\left[\left(\left(0.01 T_{\mathrm{a}}\right)-0.37\right)\left(Q_{\mathrm{s}}\right)\right] 0.000673\right\} 2.54
$$

where

PET is potential evaporation, in centimeters per day,

$\mathrm{T}_{\mathrm{a}}$ is air temperature, in degrees Fahrenheit, and

$Q_{\mathrm{s}}$ is solar radiation, in cal ories per square centimeter per day.

The units for PET can be converted to feet per day by multiplying by 0.0328 . 
By using air temperature and solar radiation from the nearby weather station (fig. 2), monthly evaporation rates were calculated with equation 3 . These estimated evaporation rates ranged from 0.04 to $0.97 \mathrm{ft} / \mathrm{mo}$ from $\mathrm{M}$ arch 2002 through December 2011 (table 1; Heilweil and others, 2005; Heilweil and Susong, 2007; Heilweil and others, 2009, Heilweil and $M$ arston, 2011). M ultiplying the estimated evaporation rates by average reservoir surface area yields monthly evaporation losses that ranged from about 20 to 1,200 acre-ft between M arch 2002 and December 2011.

\section{Estimates of Managed Aquifer Recharge from Sand Hollow Reservoir}

M onthly estimates of precipitation (P), evaporation (E), inflows $\left(\mathrm{I}_{\mathrm{sw}}\right.$ and $\left.\mathrm{I}_{\mathrm{dr}}\right)$, outflows $\left(\mathrm{O}_{\mathrm{sw}}\right)$, and changes in surfacewater storage $(\Delta S)$ were used in equation (1) to calculate recharge to the Navajo Sandstone aquifer beneath Sand Hollow Reservoir. M onthly recharge from M arch 2002 through December 2011 ranged from about 50 to 3,500 acre-ft (fig. 9 ), with two standard deviation $(\sigma)$ composite uncertainties ranging from about 6 to 14 percent of the estimate (table 1 ). Higher composite uncertainties in the summer reflect the larger, weighted importance of evaporation losses, which have the highest uncertainty. Several monthly recharge values differ from previously reported values in Heilweil and others (2005), Heilweil and Susong (2007), and Heilweil and others (2009) because of the inclusion of both " $M$ onthly drain and spring return flow to reservoir" and "Monthly outflow to Sand Hollow Resort" (both through the 60-in pipeline) in the current estimates of monthly recharge. Instances of differing values from previous reports are noted with an explanation of identified cause of error in table 1.

Estimated average monthly recharge rates beneath Sand Hollow Reservoir ranged from about 0.001 to $0.43 \mathrm{ft} / \mathrm{d}$ between March 2002 and December 2011 (fig. 10). Although the graph shows large monthly fluctuations, three general periods can be observed. Period 1 ( $M$ arch through J une 2002) had very high initial rates and then a rapid decrease as the vadose zone of the Navajo Sandstone became saturated and a hydraulic connection between the reservoir and aquifer was established, causing an abrupt decrease in hydraulic gradient. This establishment of a saturated hydraulic connection is supported by measurements in monitoring wells closest to the reservoir, which show rapidly rising water levels beginning in late spring 2002 near the southern end of the reservoir (fig. 5). Although consecutive monthly recharge rates occasionally fluctuate by more than 100 percent, Period 2 (mid-2002 through mid-2007) generally shows a gradual decline in recharge rates, while Period 3 (mid-2007 through 2011) demonstrates relatively constant, low recharge rates.

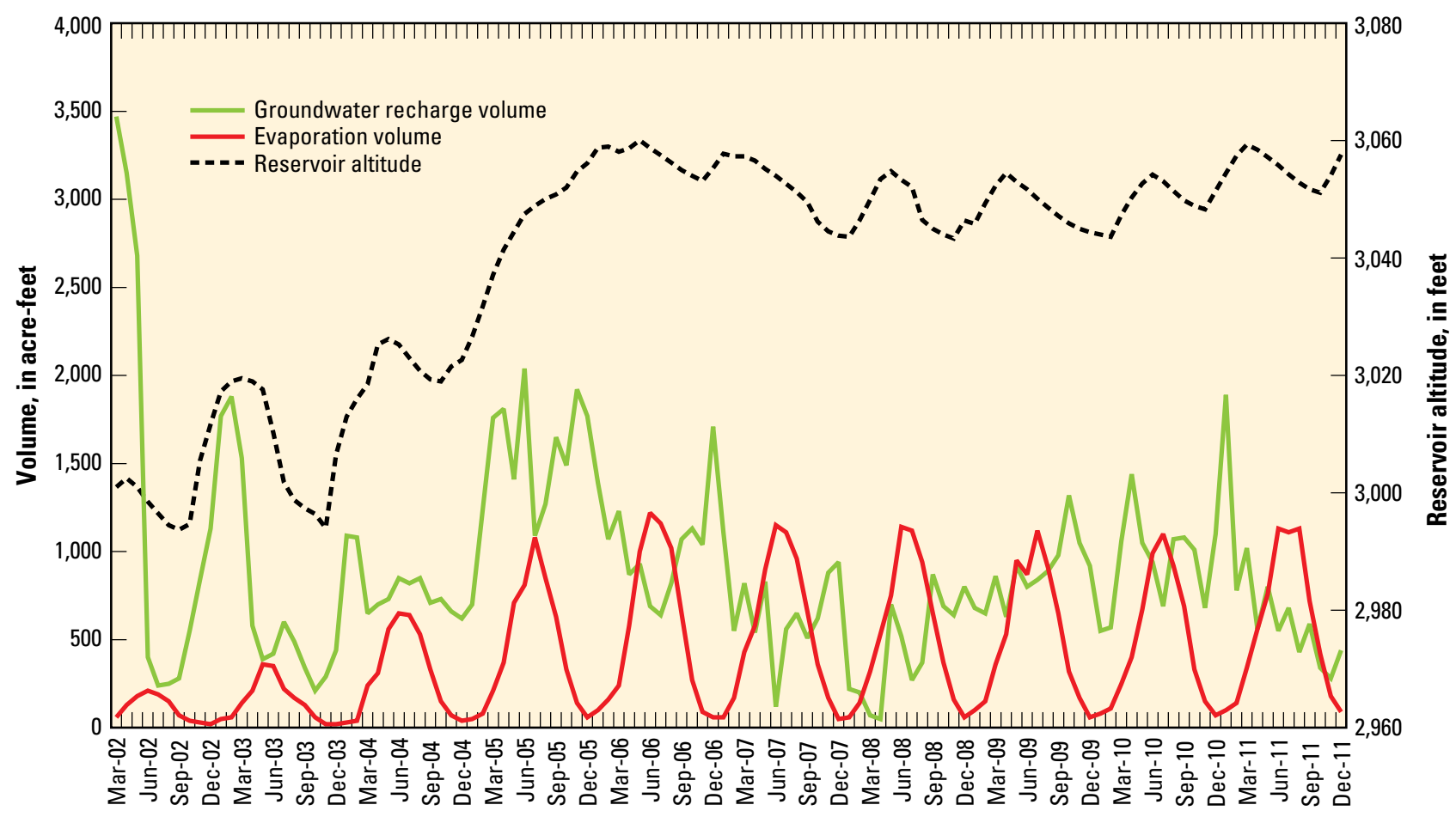

Figure 9. Monthly estimated evaporation, recharge, and reservoir altitude, Sand Hollow Reservoir, Utah, 2002-11. 
The general decline in observed recharge rates can be caused in part by (1) a decrease in the regional hydraulic gradient with rising water levels as managed aquifer recharge continues to increase groundwater storage in Sand Hollow basin, or (2) changes in permeability caused by trapped gas bubble exsolution or dissolution (Heilweil and others, 2009b), biofilm growth and decay, and silt accumulation or reduction, or both. A numerical simulation of managed aquifer recharge from Sand Hollow Reservoir to the surrounding Hurricane $B$ ench area was utilized to assess the reservoir recharge boundary in relation to the regional hydraulic gradient. Initial use of a reservoir bed conductance similar to the hydraulic conductivity of the Navajo Sandstone yielded an overestimate of recharge relative to calculated values. The model required a reduced hydraulic conductivity beneath the reservoir to match nearby water levels and calculated monthly reservoir recharge rates (M arston and Heilweil, 2012).

M onthly recharge rates, particularly during Period 2 (fig. 10), indicate seasonal fluctuations that are likely not caused by changes in hydraulic gradient. Recharge rates are generally higher during the winter months (particularly evident in 2003 through 2007), even though infiltrating water is cooler and more viscous during that time of year. A previous study at Sand Hollow (Heilweil and others, 2009) suggested biofilm growth/dissipation and summer-time bubble formation from plant respiration/decay as possible mechanisms for this variability. Further studies are needed to evaluate if these seasonal fluctuations in recharge beneath Sand Hollow Reservoir are caused by (1) a seasonal die-off of the biofilm layer and a decrease in physical clogging as this biofilm layer is broken up, (2) dissipation of the silt layer in the shallower parts of the reservoir due to wave action associated with the increase in winter/spring winds, (3) a reduction of plant-respired gases and increased solubility of biogenic gas bubbles due to cooler water temperatures, and (or) (4) the dissolution of or physical reduction in the size of trapped gas bubbles due to cooler temperatures. Previous calculations using the ideal gas law have shown that a $25^{\circ} \mathrm{C}$ reduction in reservoir water temperature from summer to winter would cause an 8 percent reduction in the volume of entrapped-gas bubbles. L aboratory permeability testing showed that a 10-percent increase in saturation resulted in an order of magnitude increase (from 0.06 to $0.6 \mathrm{ft} / \mathrm{d}$ ) in the hydraulic conductivity of sandstone cores (Heilweil and others, 2004).

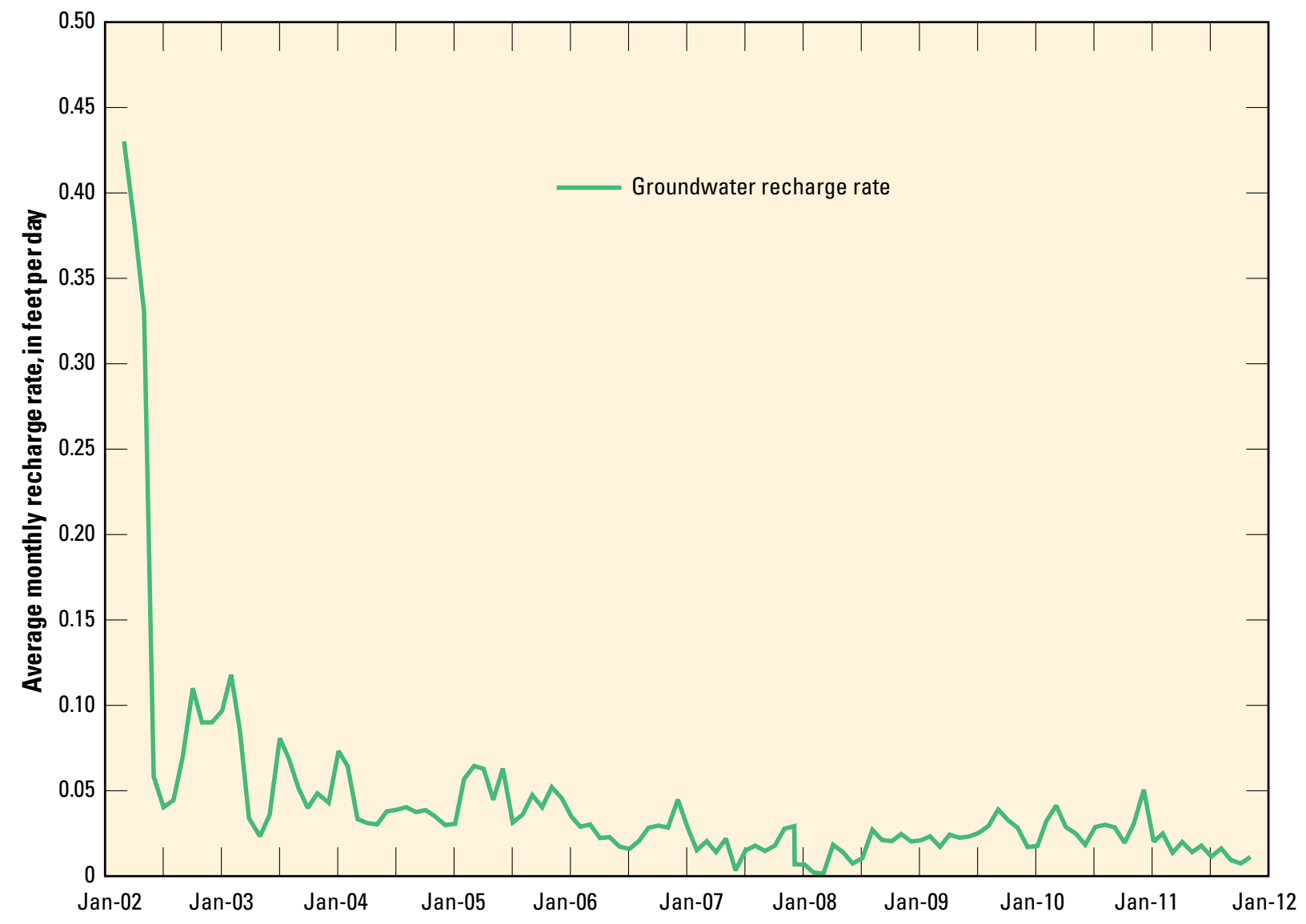

Figure 10. Monthly calculated recharge rates beneath Sand Hollow Reservoir, Utah, 2002-11. 
Net annual inflow, evaporation, and groundwater recharge from Sand Hollow Reservoir from 2002 through 2011 are shown in figure 11 . Total net inflow during this period was about 199,000 acre-ft, with annual inflow during this period ranging from about 800 acre-ft in 2007 to 56,000 acre-ft in 2005. The general increase in reservoir water-level altitude and area from 2002 to 2007 resulted in a steady increase in the volume of annual evaporation from about 1,000 acre-ft in 2002 to about 6,600 acre-ft in 2006, which then leveled off from 2007 through 2011. Total estimated evaporative losses from 2002 through 2011 were about 50,000 acre-ft. A nnual recharge ranged from a low of about 5,000 acre-ft in 2008 to a high of about 18,000 acre-ft in 2005. Total estimated recharge from 2002 through 2011 was about 106,000 acre-ft, with a two standard deviation uncertainty of 9,200 acre-ft.

\section{Evaluation of the Movement of Managed Aquifer Recharge and Geochemical Mixing in the Navajo Sandstone}

As managed aquifer recharge from Sand Hollow moves into the underlying aquifer, it has an initial water-quality signature similar to the reservoir water, but this evolves as water moves through the shallow subsurface to the pre-reservoir water-table depth within the aquifer. This water-quality signature includes field parameters, major- and trace-ion chemistry, isotopes, and dissolved gases. A long its travel path, the managed aquifer recharge water initially moves from the reservoir through the organic-rich silt layer that has accumulated beneath the reservoir, and then through the pre-reservoir vadose zone (now saturated) where vadose-zone solutes had naturally accumulated and air was trapped prior to and during filling of the reservoir. This results in an overall managed aquifer recharge water-quality signature that is distinctly different than that of native groundwater. Water-quality data from Sand Hollow Reservoir and surrounding monitoring wells, therefore, are used to assess the movement of groundwater recharge from the reservoir through the Navajo Sandstone aquifer. These include both field water-quality parameters as well as water samples collected for laboratory chemical, isotopic, and dissolved-gas analysis.

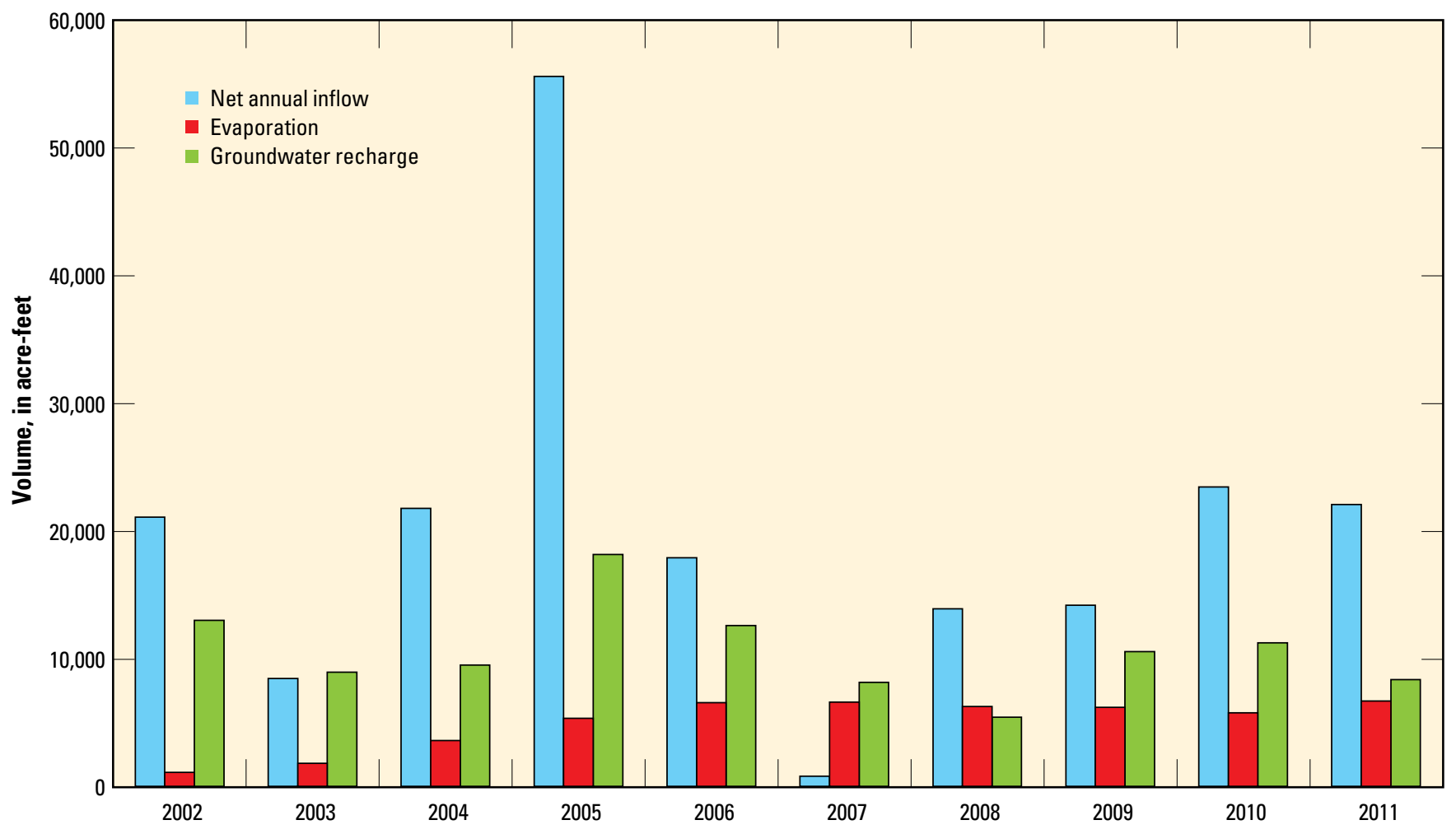

Figure 11. Estimated annual inflow, evaporation, and recharge from Sand Hollow Reservoir, Utah, 2002-11. 
Field parameters were measured to provide an on-site indication of both surface- and groundwater quality. Field water-quality parameters included water temperature, specific conductance, $\mathrm{pH}$, dissolved oxygen, and total dissolved-gas (TDG) pressure. TDG pressure is the combination of the partial pressures of all the dissolved gases in water. If dissolution of air is the primary source, these dissolved gases will be predominantly nitrogen, oxygen, and argon. Dissolved gases may also include carbon dioxide, methane, hydrogen sulfide $\left(\mathrm{CO}_{2}, \mathrm{CH}_{4}, \mathrm{H}_{2} \mathrm{~S}\right)$, and other gases formed during plant respiration and microbial consumption (anaerobic and aerobic decay). While both dissolved oxygen and TDG pressure in the recharging reservoir water are initially in atmospheric equilibrium, these parameters can increase if this water circulates through porous media with trapped air bubbles, which can form as the vadose zone becomes saturated from the wetting front beneath the reservoir connecting with the underlying aquifer (Heilweil and others, 2005). A s hydrostatic pressure increases with depth below the reservoir altitude, the trapped gas in the porous media is dissolved by the recharge water. In contrast, dissolved oxygen and TDG pressure can decrease through microbially-mediated reducing conditions associated with consumption of dissolved organic carbon.

\section{Data Collection Methods}

Field parameters were measured with a multi-parameter sonde placed within the screened interval at the bottom of each 2 -in monitoring well, and in the reservoir at water depths of approximately $2 \mathrm{ft}$. The multi-parameter sonde was too large to enter the 1-in monitoring wells ( $N$ orth Dam 3A, WD 1, WD 4, WD 5, WD RJ, and WD 12). Consequently, field measurements from these wells were made on site with a flowthrough chamber connected to the discharge lines from either a Waterra or peristaltic pump; no TDG pressure measurements were made at these sites. Additional details regarding field parameter methods are given in H eilweil and others (2005) and Heilweil and Susong (2007).

L aboratory water-quality analyses of surface water from Sand Hollow Reservoir and groundwater from the Navajo Sandstone aquifer included major and trace dissolved inorganic and organic constituents, tritium $\left({ }^{3} \mathrm{H}\right)$, and noble and industrial dissolved gases. The major inorganic ions included calcium, magnesium, sodium, potassium, bicarbonate, sulfate, chloride, fluoride, and nitrate. Trace ions included bromide, iron, manganese, arsenic, nitrite, ammonia, and orthophosphate. Organic constituents included dissolved organic carbon. Dissolved gases included chlorofluorocarbons ( $\mathrm{CFC}-11$, CFC-12, CFC-113), sulfur hexafluoride $\left(\mathrm{SF}_{6}\right)$, and noble gases ( $\mathrm{He}, \mathrm{Ne}, \mathrm{Ar}, \mathrm{Kr}, \mathrm{Xe}$ ).

Water-chemistry samples were collected from 2 -in monitoring wells by using either a Grunfos or B ennett sample pump; 1-in wells were sampled with a Waterra hand pump; production wells were sampled utilizing installed turbine pumps. Prior to water-chemistry sample collection from monitoring wells, water was purged from each well until field parameters stabilized and a minimum of three casing volumes were removed. A fter purging each well, water was pumped into samples bottles and filtered as necessary. Since 2009, a set of replicates for all constituents has been separately analyzed annually at one randomly selected sampling site for qual ity assurance.

Samples for major and trace ions were filtered with 0.45 -micron disposable filters and collected in cleaned polyethylene bottles according to procedures described by Wilde and Radtke (1998); samples for cation analysis were preserved with 7.7-normal nitric acid. Tritium samples were collected in 500 milliliter (ml) polyethylene bottles with polyseal caps without head space. CFC and $\mathrm{SF}_{6}$ samples were collected in 250-ml and 1-liter (L) glass bottles, respectively, according to procedures described at: http://water.usgs.gov/lab/. Noble gases were collected using diffusion sampler methods described by Sheldon (2002) and Gardner and Solomon (2009).

Inorganic and organic chemical analyses (major ions, trace ions, dissolved organic carbon) were analyzed by the U.S. Geological Survey at the National Water Quality Laboratory in Denver, Colorado. CFCs and $\mathrm{SF}_{6}$ were analyzed by the U.S. Geological Survey at the Chlorofluorocarbon Laboratory in Reston, Virginia. Tritium and noble gases were analyzed by the U niversity of U tah's Dissolved Gas Service Center using quadrupole and sector-field mass spectrometers; tritium concentrations were determined by the in-growth method (Clarke and others, 1976).

\section{Water-Quality Results}

\section{Field Parameters}

Total dissolved-gas (TDG) pressures at the three 2-in monitoring wells closest to the reservoir (WD 6, WD 9, WD 11) have shown the arrival and passage of peak values associated with reservoir recharge. TDG pressures at these wells increased from background values of 700 to $850 \mathrm{mil}$ limeters of mercury $(\mathrm{mm} \mathrm{Hg})$ to values of 1,800 to more than $2,250 \mathrm{~mm} \mathrm{Hg}$, or about two to three times atmospherically equilibrated concentrations. During 2011-2012, TDG pressures remained el evated at WD 6, but have declined at WD 9 and WD 11 (fig. 12). The multi-parameter sonde used for TDG pressure measurements relies on a pressure transducer that cannot measure pressures greater than $2,250 \mathrm{~mm} \mathrm{Hg}$ and is not within its linear calibration range above about $1,500 \mathrm{~mm} \mathrm{Hg}$. $M$ easurements less than $1,500 \mathrm{~mm} \mathrm{Hg}$ have an error of less than 5 percent. B ecause of this non-linearity of in-situ measurements greater than $1,500 \mathrm{~mm} \mathrm{Hg}$, analyses of noble gases collected with advanced diffusion samplers (Gardner and Solomon, 2009) have been used in selected monitoring wells for more accurate TDG pressures (see footnote 2 of table 2). M easured TDG pressure values at WD 9 (located $55 \mathrm{ft}$ from the 


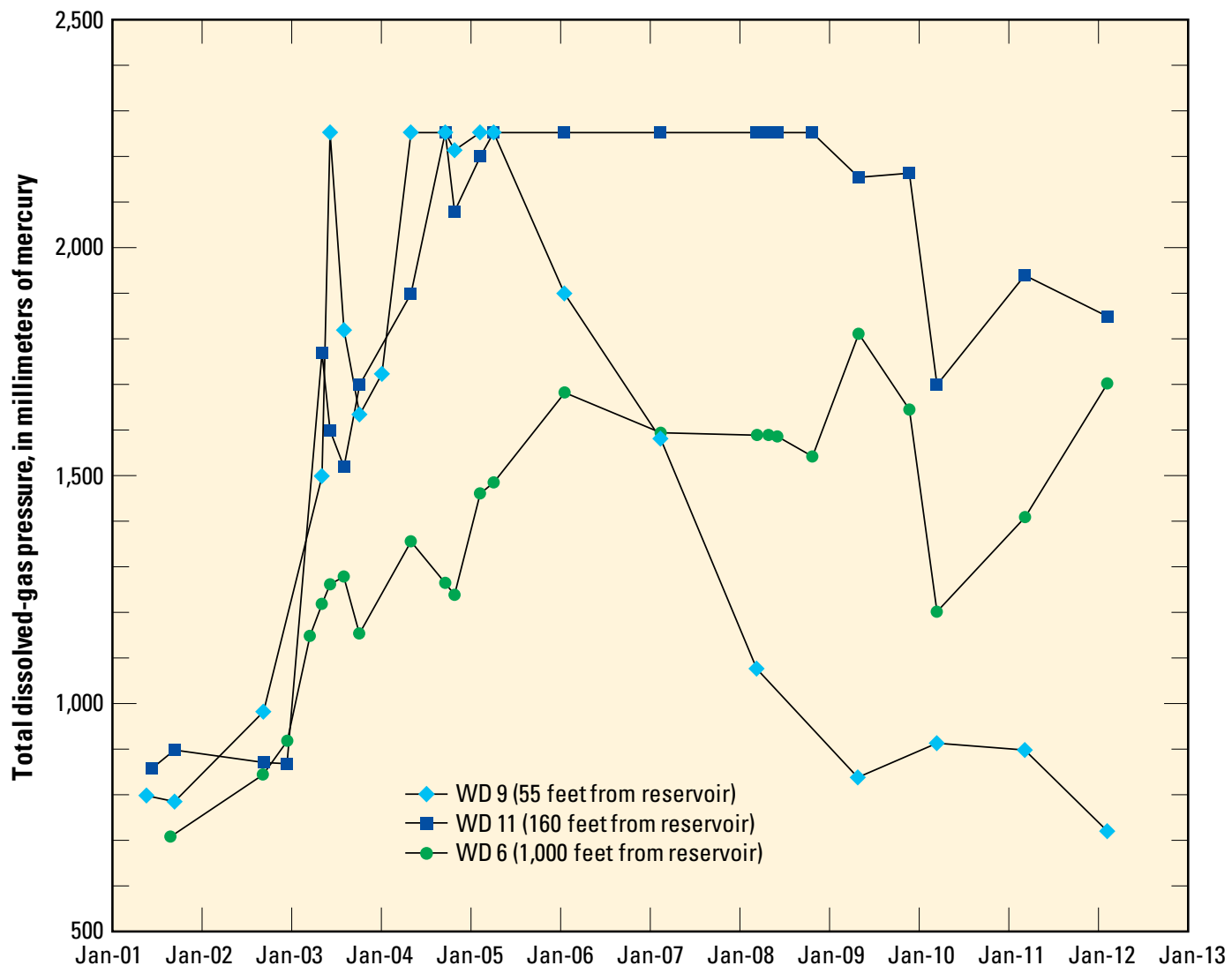

Figure 12. Total dissolved-gas pressure in groundwater from selected monitoring wells in Sand Hollow, Utah, 2001-2012.

reservoir) exceeded 2,250 mm $\mathrm{Hg}$ during February and April 2005 , indicating peak arrival about 3 years after inception of the reservoir. TDG pressures at WD 9 have since declined to pre-reservoir conditions - the 2012 measurement of $720 \mathrm{~mm}$ $\mathrm{Hg}$ was only slightly higher than the measured reservoir TDG and local barometric pressure of about $700 \mathrm{~mm} \mathrm{Hg}$. TDG pressures measured at WD 11 (located $160 \mathrm{ft}$ from the reservoir) exceeded 2,250 $\mathrm{mm} \mathrm{Hg}$ from 2005 through 2008, so an exact peak-arrival date could not be determined. Since 2010, TDG pressures declined slightly, fluctuating between about 1,700 and $1,900 \mathrm{~mm} \mathrm{Hg}$. TDG pressure measurements at WD 6 (located 1,000 ft from the reservoir) reached a peak of about $1,800 \mathrm{~mm} \mathrm{Hg}$ in A pril 2009 and declined to $1,200 \mathrm{~mm} \mathrm{Hg}$ in 2010, but increased back up to $1,700 \mathrm{~mm} \mathrm{Hg}$ in 2012. These fluctuations can be caused by changing hydraulic conditions associated with nearby pumping at Well 8 . Of the monitoring wells drilled in 2008 (WD 15 through WD 20), elevated TDG pressures have only been measured at WD $15(1,300-1,500$ $\mathrm{mm} \mathrm{Hg}$ ), located closer to the reservoir and screened shallower in the aquifer (where water-level changes have been larger) than WD 16 through WD 20. These el evated TD G pressures were likely caused by rising water levels and entrapment of air bubbles in the shallow part of the aquifer at this location rather than signifying the arrival of reservoir recharge.

Dissolved-oxygen (DO) concentrations at the three 2-in monitoring wells closest to the reservoir (WD 6, WD 9,
WD 11) showed the arrival and passage of peak values associated with reservoir recharge (table 2 ). B ackground native groundwater $D 0$ values were generally between 6.1 and $8.7 \mathrm{mg} / \mathrm{L}$. Similar to TDG pressure, DO reached elevated values, from about 18 to $26 \mathrm{mg} / \mathrm{L}$ (about 2 to 3 times atmospheric equilibration), in monitoring wells near the reservoir. DO at both WD 9 and WD 11 reached maximum values in A pril 2005; D 0 peaked at WD 6 in A pril 2009. W hile DO values have since declined at all three sites, WD 9 showed a much sharper decline, and had values of less than $0.5 \mathrm{mg} / \mathrm{L}$ in 2011 and 2012. These values were much less than DO measurements of reservoir water (ranging from 7 to $12 \mathrm{mg} / \mathrm{L}$ ) and likely indicate biological consumption of oxygen in the shallow organic-rich sediments beneath the reservoir.

Prior to inception of Sand Hollow Reservoir in M arch 2002, specific-conductance values of native groundwater ranged from $130 \mu \mathrm{S} / \mathrm{cm}$ at WD 6 to $560 \mu \mathrm{S} / \mathrm{cm}$ at WD RJ (table 3, fig. 13). Elevated specific-conductance values at four monitoring wells (N orth Dam 3A, WD 6, WD 9, and WD 11) indicate the arrival of reservoir recharge. A these sites, specific-conductance values all peaked at values greater than those of the reservoir, then declined to values similar to the reservoir. This higher peak is attributed to the mobilization of natural salts that accumulated in the vadose zone prior to the inception of the reservoir and were incorporated into the leading edge of the reservoir recharge. These peak arrivals indicate 
Table 2. Field water-quality parameters, dissolved organic carbon, tritium, chlorofluorocarbons, sulfur hexafluoride, and neon excess in groundwater and surface water from Sand Hollow, Utah.

[Analyzing agency: Dissolved organic carbon at U.S. G eological Survey (USGS) National Water Quality Laboratory in Denver, Colorado; Tritium at U niversity of Utah. Dissolved Gas Laboratory in Salt Lake City, Utah; CFC-11, CFC-12, CFC-113, SF 6 at USGS Chlorofluorocarbon (CFC) Laboratory in Reston, Virginia. Water temperature: ${ }^{\circ} \mathrm{C}$, degrees Celsius; Specific conductance: $\mu \mathrm{S} / \mathrm{cm}$, microsiemens per centimeter at 25 degrees Celsius; Dissolved oxygen, dissolved organic carbon: $\mathrm{mg} / \mathrm{L}$, milligrams per liter; Total dissolved-gas pressure: $\mathrm{mm} \mathrm{Hg}$, millimeters of mercury; Tritium, Tritium precision: TU, tritium units; CFC-11, CFC-12, CFC-113: pmol/kg, picomoles per kilogram; $\mathbf{S F}_{6}$ : fmol/kg, femtomoles per kilogram; -, no data available; E, estimated; >, greater than; <, less than]

\begin{tabular}{|c|c|c|c|c|c|c|c|c|c|c|c|c|c|c|}
\hline Site name & $\begin{array}{c}\text { Date } \\
\text { sampled }\end{array}$ & $\begin{array}{l}\text { Water tem- } \\
\text { perature } \\
\left({ }^{\circ} \mathrm{C}\right)\end{array}$ & $\begin{array}{c}\text { Specific } \\
\text { conduc- } \\
\text { tance } \\
(\mu \mathrm{S} / \mathrm{cm})\end{array}$ & $\begin{array}{c}\mathrm{pH} \\
\text { (standard } \\
\text { units) }\end{array}$ & $\begin{array}{c}\text { Dissolved } \\
\text { oxygen } \\
\text { (mg/L) }\end{array}$ & $\begin{array}{c}\text { Total dis- } \\
\text { solved-gas } \\
\text { pressure } \\
(\mathrm{mm} \mathrm{Hg})\end{array}$ & $\begin{array}{c}\text { Dissolved } \\
\text { organic } \\
\text { carbon } \\
\text { (mg/L) }\end{array}$ & $\begin{array}{c}\text { Tritium } \\
\text { (TU) }\end{array}$ & $\begin{array}{l}\text { Tritium } \\
\text { precision } \\
\text { (TU) }\end{array}$ & $\begin{array}{c}\text { CFC-11 } \\
\text { (pmole/ } \\
\mathrm{kg})\end{array}$ & $\begin{array}{c}\text { CFC-12 } \\
\text { (pmole/ } \\
\text { kg) }\end{array}$ & $\begin{array}{c}\text { CFC-113 } \\
\text { (pmole/ } \\
\text { kg) }\end{array}$ & $\begin{array}{c}\mathrm{SF}_{6} \\
(\mathbf{f m o l} / / \\
\mathbf{k g})\end{array}$ & $\begin{array}{c}\text { Neon } \\
\text { excess } \\
\text { (percent) }\end{array}$ \\
\hline \multicolumn{15}{|c|}{ Native groundwater } \\
\hline \multirow[t]{10}{*}{ North Dam $3 \mathrm{~A}$} & $10 / 8 / 2002$ & 15.9 & 4,430 & 8.0 & 5.0 & - & - & 2.71 & 0.14 & - & - & - & - & - \\
\hline & $12 / 18 / 2002$ & 14.7 & 2,830 & 8.0 & 10.8 & - & - & - & - & - & - & - & - & - \\
\hline & $6 / 10 / 2003$ & 21.5 & 1,330 & 7.8 & - & - & - & - & - & - & - & - & - & - \\
\hline & $9 / 21 / 2004$ & 18.4 & 980 & 7.7 & 11.0 & - & - & - & - & - & - & - & - & - \\
\hline & $10 / 29 / 2004$ & 15.9 & 910 & 7.9 & 11.1 & - & - & - & - & - & - & - & - & - \\
\hline & $2 / 10 / 2005$ & 15.3 & 960 & 7.7 & 13.5 & - & - & - & - & - & - & - & - & - \\
\hline & $4 / 5 / 2005$ & 16.5 & 960 & 7.8 & 12.6 & - & - & - & - & - & - & - & - & - \\
\hline & $1 / 19 / 2006$ & - & 840 & 8.0 & - & - & - & - & - & - & - & - & - & - \\
\hline & $3 / 10 / 2011$ & 20.3 & 830 & 7.4 & 0.8 & - & 1.93 & 2.87 & 0.12 & 0.60 & 1.93 & 0.09 & - & - \\
\hline & $2 / 6 / 2012$ & 11.3 & 820 & 7.8 & - & - & 2.00 & 3.46 & 0.41 & - & - & - & - & - \\
\hline \multirow[t]{9}{*}{ WD 4} & 4/2/1999 & 21.0 & 360 & 8.2 & - & - & - & 0.22 & 0.10 & - & - & - & - & - \\
\hline & $12 / 18 / 2002$ & 18.7 & 350 & 7.7 & 8.1 & - & - & - & - & - & - & - & - & - \\
\hline & $1 / 19 / 2006$ & - & 350 & 8.0 & - & - & - & - & - & - & - & - & - & - \\
\hline & $2 / 15 / 2007$ & 19.0 & 340 & 7.9 & 8.7 & - & - & - & - & - & - & - & - & - \\
\hline & $3 / 13 / 2008$ & 22.6 & 350 & 7.8 & 7.8 & - & - & 0.25 & 0.10 & - & - & - & - & - \\
\hline & $10 / 23 / 2008$ & 21.2 & 360 & 8.0 & - & - & - & 0.13 & 0.10 & 0.62 & 0.61 & 0.09 & 0.44 & - \\
\hline & $4 / 28 / 2009$ & - & 350 & 7.8 & - & - & - & 0.15 & 0.07 & 0.54 & 0.52 & 0.07 & 0.45 & - \\
\hline & $11 / 24 / 2009$ & 18.7 & 340 & 7.8 & 9.5 & - & 0.43 & 0.09 & 0.03 & 0.42 & 0.54 & 0.07 & - & - \\
\hline & $3 / 15 / 2010$ & 19.7 & 360 & 7.7 & 9.5 & - & E0.37 & 0.06 & 0.03 & 0.62 & 0.60 & 0.09 & - & - \\
\hline \multirow{5}{*}{ WD 5} & $10 / 23 / 2008$ & 21.0 & 540 & 8.2 & - & - & - & 0.07 & 0.10 & 0.12 & 0.08 & 0.01 & 0.13 & - \\
\hline & $4 / 30 / 2009$ & - & 520 & 7.5 & - & - & - & 0.02 & 0.06 & - & - & - & - & - \\
\hline & $11 / 24 / 2009$ & 16.9 & 510 & 8.5 & 7.2 & - & 0.45 & 0.09 & 0.05 & 0.20 & 0.07 & 0.02 & - & - \\
\hline & $3 / 15 / 2010$ & 21.0 & 540 & 7.7 & 8.1 & - & E 0.44 & 0.09 & 0.10 & 0.19 & 0.10 & 0.03 & - & - \\
\hline & $3 / 10 / 2011$ & 19.5 & 510 & 7.4 & 8.0 & - & 0.72 & 0.00 & 0.03 & 0.12 & 0.07 & 0.03 & 0.10 & - \\
\hline \multirow[t]{8}{*}{ WD RJ } & 4/2/1999 & 18.0 & 560 & 8.2 & - & - & - & 0.02 & 0.05 & - & - & - & - & - \\
\hline & $12 / 17 / 2002$ & 18.2 & 530 & 7.7 & 6.4 & - & - & - & - & - & - & - & - & - \\
\hline & $1 / 18 / 2006$ & - & 550 & 7.7 & - & - & - & - & - & - & - & - & - & - \\
\hline & $2 / 15 / 2007$ & 19.0 & 530 & 7.7 & 8.1 & - & - & - & - & - & - & - & - & - \\
\hline & $3 / 12 / 2008$ & 19.3 & 540 & 7.3 & 6.8 & - & - & 0.03 & 0.10 & - & - & - & - & - \\
\hline & $4 / 28 / 2009$ & - & 550 & 7.5 & - & - & - & 0.04 & 0.02 & - & - & - & - & - \\
\hline & $3 / 15 / 2010$ & 19.6 & 560 & 7.6 & 8.0 & - & 0.84 & 0.06 & 0.03 & 0.25 & 0.13 & 0.07 & - & - \\
\hline & $3 / 9 / 2011$ & 19.6 & 540 & 7.3 & 7.5 & - & - & 0.00 & 0.03 & 0.34 & 0.15 & 0.06 & - & - \\
\hline
\end{tabular}


Table 2. Field water-quality parameters, dissolved organic carbon, tritium, chlorofluorocarbons, sulfur hexafluoride, and neon excess in groundwater and surface water from Sand Hollow, Utah.-Continued

[Analyzing agency: Dissolved organic carbon at U.S. Geological Survey (USGS) N ational Water Quality Laboratory in Denver, Colorado; Tritium at University of U tah. Dissolved G as Laboratory in Salt Lake City, Utah; CFC-11, CFC-12, CFC-113, SF at USGS Chlorofluorocarbon (CFC) Laboratory in Reston, Virginia. Water temperature: ${ }^{\circ} \mathrm{C}$, degrees Celsius; Specific conductance: $\mu \mathrm{S} / \mathrm{cm}$, microsiemens per centimeter at 25 degrees Celsius; Dissolved oxygen, dissolved organic carbon: mg/L, milligrams per liter; Total dissolved-gas

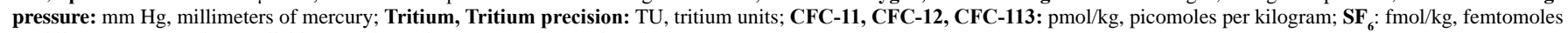
per kilogram; - , no data available; E, estimated; >, greater than; <, less than]

\begin{tabular}{|c|c|c|c|c|c|c|c|c|c|c|c|c|c|c|}
\hline Site name & $\begin{array}{c}\text { Date } \\
\text { sampled }\end{array}$ & $\begin{array}{l}\text { Water tem- } \\
\text { perature } \\
\left({ }^{\circ} \mathrm{C}\right)\end{array}$ & $\begin{array}{c}\text { Specific } \\
\text { conduc- } \\
\text { tance } \\
(\mu \mathrm{S} / \mathrm{cm})\end{array}$ & $\begin{array}{c}\mathrm{pH} \\
\text { (standard } \\
\text { units) }\end{array}$ & $\begin{array}{c}\text { Dissolved } \\
\text { oxygen } \\
\text { (mg/L) }\end{array}$ & $\begin{array}{c}\text { Total dis- } \\
\text { solved-gas } \\
\text { pressure } \\
(\mathrm{mm} \mathrm{Hg})\end{array}$ & $\begin{array}{c}\text { Dissolved } \\
\text { organic } \\
\text { carbon } \\
\text { (mg/L) }\end{array}$ & $\begin{array}{l}\text { Tritium } \\
\text { (TU) }\end{array}$ & $\begin{array}{l}\text { Tritium } \\
\text { precision } \\
\text { (TU) }\end{array}$ & $\begin{array}{c}\text { CFC-11 } \\
\text { (pmole/ } \\
\text { kg) }\end{array}$ & $\begin{array}{c}\text { CFC-12 } \\
\text { (pmole/ } \\
\text { kg) }\end{array}$ & $\begin{array}{c}\text { CFC-113 } \\
\text { (pmole/ } \\
\text { kg) }\end{array}$ & $\begin{array}{c}\mathrm{SF}_{6} \\
(\mathrm{fmol} / / \\
\mathbf{k g})\end{array}$ & $\begin{array}{c}\text { Neon } \\
\text { excess } \\
\text { (percent) }\end{array}$ \\
\hline \multirow[t]{17}{*}{ WD 6} & $5 / 15 / 2001$ & - & 130 & 7.6 & - & - & - & 4.77 & 0.24 & - & - & - & - & - \\
\hline & $9 / 9 / 2002$ & 19.4 & 290 & 7.7 & - & 850 & - & - & - & - & - & - & - & 13.5 \\
\hline & $12 / 17 / 2002$ & 19.0 & 400 & 7.6 & 9.3 & 920 & - & - & - & - & - & - & - & - \\
\hline & $6 / 9 / 2003$ & 19.6 & 390 & 7.8 & 14.0 & 1,260 & - & - & - & - & - & - & - & - \\
\hline & $8 / 4 / 2003$ & 19.3 & 350 & 7.5 & 11.9 & 1,280 & - & - & - & - & - & - & - & - \\
\hline & $10 / 6 / 2003$ & 19.6 & 400 & 7.6 & 12.0 & 1,160 & - & - & - & - & - & - & - & - \\
\hline & $5 / 3 / 2004$ & 19.4 & 700 & 7.4 & 15.2 & 1,357 & - & - & - & - & - & - & - & - \\
\hline & $9 / 20 / 2004$ & 19.6 & 820 & 7.7 & 15.0 & 1,266 & - & - & - & - & - & - & - & 75.8 \\
\hline & $2 / 15 / 2007$ & 19.1 & 1,110 & 7.6 & 17.2 & ${ }^{1} 1,600$ & - & - & - & - & - & - & - & - \\
\hline & $3 / 13 / 2008$ & 19.2 & 1,300 & 7.5 & 14.4 & ${ }^{1} 1,590$ & - & 2.11 & 0.14 & - & - & - & - & 125.3 \\
\hline & $4 / 29 / 2008$ & 19.3 & 1,290 & 7.7 & 17.1 & ${ }^{1} 1,590$ & - & - & - & - & - & - & - & 124.0 \\
\hline & $6 / 3 / 2008$ & 19.4 & 1,330 & 7.6 & 16.5 & ${ }^{1} 1,590$ & - & - & - & - & - & - & - & 124.3 \\
\hline & $10 / 24 / 2008$ & 19.0 & 1,190 & - & 16.3 & ${ }^{1} 1,540$ & - & 2.55 & 0.13 & 2.8 & 1.3 & 0.15 & 0.72 & - \\
\hline & $4 / 30 / 2009$ & 19.2 & 1,050 & 7.7 & 22.0 & ${ }^{1} 1,810$ & - & 2.66 & 0.14 & 3.2 & 1.5 & 0.16 & 0.73 & 161.7 \\
\hline & $11 / 23 / 2009$ & 18.9 & 970 & 7.9 & 15.3 & ${ }^{1} 1,650$ & 1.71 & 2.93 & 0.23 & 1.7 & 1.8 & 0.17 & - & 140.8 \\
\hline & $3 / 15 / 2010$ & 19.2 & 920 & 7.5 & 14.4 & 1,200 & 1.68 & 3.15 & 0.15 & 1.7 & 1.6 & 0.19 & - & 88.5 \\
\hline & $3 / 9 / 2011$ & 19.1 & 900 & 7.5 & 11.2 & ${ }^{2} 1,410$ & 1.56 & 1.54 & 0.10 & 2.64 & 1.73 & 0.20 & 0.65 & 125 \\
\hline \multirow[t]{2}{*}{ (replicate) } & $3 / 9 / 2011$ & 19.1 & 870 & 7.9 & - & - & 1.59 & 2.83 & 0.13 & 2.64 & 1.73 & 0.20 & 0.72 & - \\
\hline & 2/7/2012 & 19.1 & 810 & 7.3 & 15.6 & ${ }^{1} 1,700$ & 1.70 & 2.88 & 0.31 & 2.23 & 1.67 & 0.18 & 0.87 & 199 \\
\hline \multirow[t]{15}{*}{ WD 9} & $5 / 23 / 2001$ & 19.5 & 300 & 7.7 & 8.0 & 800 & - & 0.00 & 0.01 & - & - & - & - & - \\
\hline & $9 / 14 / 2001$ & 19.4 & 280 & 7.4 & - & 790 & - & 0.20 & 0.15 & - & - & - & - & 49.5 \\
\hline & $9 / 11 / 2002$ & 19.5 & 350 & 7.9 & - & 980 & - & - & - & - & - & - & - & 28.1 \\
\hline & $5 / 7 / 2003$ & 19.7 & 320 & 7.8 & - & ${ }^{1}>2,250$ & - & - & - & - & - & - & - & - \\
\hline & $6 / 9 / 2003$ & 19.5 & 350 & 7.7 & 24.4 & ${ }^{1}>2,250$ & - & - & - & - & - & - & - & 158.8 \\
\hline & $8 / 5 / 2003$ & 19.7 & 720 & 7.5 & 19.3 & ${ }^{1} 1,800$ & - & - & - & - & - & - & - & - \\
\hline & $10 / 7 / 2003$ & 19.6 & 740 & 7.5 & 17.9 & ${ }^{1} 1,600$ & - & - & - & - & - & - & - & - \\
\hline & $1 / 6 / 2004$ & 19.4 & 630 & 7.7 & 16.7 & ${ }^{1} 1,700$ & - & - & - & - & - & - & - & - \\
\hline & $5 / 3 / 2004$ & 19.4 & 530 & 7.4 & 25.7 & ${ }^{1}>2,250$ & - & - & - & - & - & - & - & - \\
\hline & $9 / 20 / 2004$ & 18.5 & 750 & 7.8 & 22.6 & ${ }^{1}>2,250$ & - & - & - & - & - & - & - & - \\
\hline & $10 / 28 / 2004$ & 18.5 & 760 & 7.6 & 20.7 & 12,210 & - & - & - & - & - & - & - & - \\
\hline & $2 / 9 / 2005$ & 18.4 & 880 & 7.7 & 20.2 & ${ }^{1}>2,250$ & - & - & - & - & - & - & - & 246.2 \\
\hline & $4 / 5 / 2005$ & 18.5 & 820 & 7.4 & 23.2 & ${ }^{1}>2,250$ & - & - & - & - & - & - & - & - \\
\hline & $1 / 18 / 2006$ & 18.0 & 1,230 & 7.9 & 15.0 & ${ }^{1} 1,900$ & - & - & - & - & - & - & - & - \\
\hline & $2 / 14 / 2007$ & 17.3 & 790 & 7.4 & 4.6 & ${ }^{1} 1,600$ & - & - & - & - & - & - & - & - \\
\hline
\end{tabular}


Table 2. Field water-quality parameters, dissolved organic carbon, tritium, chlorofluorocarbons, sulfur hexafluoride, and neon excess in groundwater and surface water from Sand Hollow, Utah.-Continued

[Analyzing agency: Dissolved organic carbon at U.S. G eological Survey (USGS) N ational Water Quality Laboratory in Denver, Colorado; Tritium at U niversity of U tah. Dissolved Gas L aboratory in Salt L ake City, U tah; CFC-11, CFC-12, CFC-113, SF ${ }_{6}$ at USGS Chlorofluorocarbon (CFC) Laboratory in Reston, Virginia. Water temperature: ${ }^{\circ} \mathrm{C}$, degrees Celsius; Specific conductance: $\mu \mathrm{S} / \mathrm{cm}$, microsiemens per centimeter at 25 degrees Celsius; Dissolved oxygen, dissolved organic carbon: $\mathrm{mg} / \mathrm{L}$, milligrams per liter; Total dissolved-gas pressure: $\mathrm{mm} \mathrm{Hg}$, millimeters of mercury; Tritium, Tritium precision: TU, tritium units; CFC-11, CFC-12, CFC-113: pmol/kg, picomoles per kilogram; $\mathbf{S F}_{6}$ : fmol/kg, femtomoles per kilogram; -, no data available; E, estimated; >, greater than; <, less than]

\begin{tabular}{|c|c|c|c|c|c|c|c|c|c|c|c|c|c|c|}
\hline Site name & $\begin{array}{c}\text { Date } \\
\text { sampled }\end{array}$ & $\begin{array}{c}\text { Water tem- } \\
\text { perature } \\
\left({ }^{\circ} \mathrm{C}\right)\end{array}$ & $\begin{array}{c}\begin{array}{c}\text { Specific } \\
\text { conduc- } \\
\text { tance } \\
(\mu \mathrm{S} / \mathrm{cm})\end{array}\end{array}$ & $\begin{array}{c}\mathrm{pH} \\
\text { (standard } \\
\text { units) }\end{array}$ & $\begin{array}{c}\text { Dissolved } \\
\text { oxygen } \\
\text { (mg/L) }\end{array}$ & $\begin{array}{c}\text { Total dis- } \\
\text { solved-gas } \\
\text { pressure } \\
(\mathrm{mm} \mathrm{Hg})\end{array}$ & $\begin{array}{c}\text { Dissolved } \\
\text { organic } \\
\text { carbon } \\
\text { (mg/L) }\end{array}$ & $\begin{array}{c}\text { Tritium } \\
\text { (TU) }\end{array}$ & $\begin{array}{l}\text { Tritium } \\
\text { precision } \\
\text { (TU) }\end{array}$ & $\begin{array}{c}\text { CFC-11 } \\
\text { (pmole/ } \\
\text { kg) }\end{array}$ & $\begin{array}{c}\text { CFC-12 } \\
\text { (pmole/ } \\
\mathrm{kg})\end{array}$ & $\begin{array}{c}\text { CFC-113 } \\
\text { (pmole/ } \\
\mathrm{kg})\end{array}$ & $\begin{array}{c}\mathbf{S F}_{6} \\
(\mathbf{f m o l} / \\
\mathbf{k g})\end{array}$ & $\begin{array}{c}\text { Neon } \\
\text { excess } \\
\text { (percent) }\end{array}$ \\
\hline \multirow[t]{2}{*}{ WD 9} & $3 / 11 / 2008$ & 17.0 & 820 & 7.3 & 1.5 & 1,080 & - & 2.61 & 0.22 & - & - & - & - & 138.4 \\
\hline & $3 / 15 / 2010$ & 16.4 & 840 & 7.3 & 1.7 & 920 & 1.23 & 3.20 & 0.14 & 0.8 & 2.2 & 0.21 & - & 103.4 \\
\hline (replicate) & $3 / 15 / 2010$ & 16.4 & 840 & 7.3 & 1.7 & 920 & 1.17 & 2.90 & 0.12 & 0.8 & 2.2 & 0.18 & - & - \\
\hline \multirow[t]{16}{*}{ WD 11} & $6 / 14 / 2001$ & 18.5 & 420 & 7.8 & 8.1 & 860 & - & - & - & - & - & - & - & - \\
\hline & $9 / 14 / 2001$ & 18.5 & 450 & 7.7 & 8.6 & 900 & - & 0.53 & 0.08 & 0.53 & 0.24 & - & - & 70.9 \\
\hline & $9 / 12 / 2002$ & 18.5 & 465 & 7.6 & - & 873 & - & - & - & - & - & - & - & 26.8 \\
\hline & $12 / 16 / 2002$ & 18.2 & 455 & 7.6 & 8.1 & 890 & - & - & - & - & - & - & - & - \\
\hline & $5 / 7 / 2003$ & 18.4 & 620 & 7.7 & - & ${ }^{1} 1,770$ & - & - & - & - & - & - & - & - \\
\hline & $9 / 20 / 2004$ & 18.0 & 920 & 8.2 & 23.5 & ${ }^{1}>2,250$ & - & - & - & - & - & - & - & - \\
\hline & $10 / 28 / 2004$ & 18.0 & 990 & 7.9 & 22.8 & 12,080 & - & - & - & - & - & - & - & - \\
\hline & $2 / 9 / 2005$ & 18.0 & 960 & 8.1 & 22.1 & 12,200 & - & - & - & - & - & - & - & 162.5 \\
\hline & $4 / 5 / 2005$ & 17.8 & 930 & 7.9 & 25.2 & ${ }^{1}>2,250$ & - & - & - & - & - & - & - & - \\
\hline & $1 / 18 / 2006$ & 17.6 & 980 & 7.9 & 23.0 & ${ }^{1}>2,250$ & - & - & - & - & - & - & - & - \\
\hline & $2 / 14 / 2007$ & 17.1 & 820 & 7.6 & 19.0 & ${ }^{1}>2,250$ & - & - & - & - & - & - & - & - \\
\hline & $3 / 11 / 2008$ & 17.0 & 840 & 7.6 & 14.9 & ${ }^{1}>2,250$ & - & 2.30 & 0.14 & - & - & - & - & 319.1 \\
\hline & $4 / 30 / 2008$ & 17.0 & 840 & 7.7 & 17.4 & ${ }^{1}>2,250$ & - & - & - & - & - & - & - & - \\
\hline & $6 / 2 / 2008$ & 17.1 & 850 & 7.7 & 18.9 & ${ }^{1>2,250}$ & - & - & - & - & - & - & - & 213.8 \\
\hline & $10 / 22 / 2008$ & 16.7 & 840 & 8.0 & 15.9 & ${ }^{1}>2,250$ & - & 2.36 & 0.11 & - & - & - & - & - \\
\hline & $4 / 30 / 2009$ & 15.9 & 840 & 7.7 & 19.4 & 12,160 & - & 3.06 & 0.14 & 2.0 & 3.0 & 0.34 & 3.5 & 291.3 \\
\hline \multirow{2}{*}{ WD 12} & $3 / 9 / 2011$ & 19.9 & 1,670 & 7.1 & 13.4 & - & 2.17 & 0.96 & 0.06 & 2.34 & 2.20 & 0.25 & 0.68 & - \\
\hline & 2/8/2012 & 19.3 & 2,100 & 7.2 & 9.2 & - & 3.81 & 1.01 & 0.13 & 2.27 & 2.04 & 0.28 & 1.10 & - \\
\hline \multirow[t]{6}{*}{ WD 15} & $10 / 25 / 2008$ & 18.8 & 720 & - & 14.2 & 1,300 & - & - & - & - & - & - & - & - \\
\hline & $4 / 28 / 2009$ & 18.9 & 710 & 8.0 & 17.6 & 1,490 & - & 0.77 & 0.04 & 2.3 & 1.9 & 0.23 & 1.4 & 92.9 \\
\hline & $11 / 23 / 2009$ & 18.8 & 730 & 8.3 & 14.5 & 1,410 & 2.47 & 0.68 & 0.05 & 1.0 & 1.9 & 0.22 & - & 88.5 \\
\hline & $3 / 16 / 2010$ & 19.1 & 730 & 7.9 & 11.5 & 1,320 & 2.49 & 0.72 & 0.05 & 1.2 & 2.1 & 0.25 & - & 73.7 \\
\hline & $3 / 8 / 2011$ & 19.1 & 820 & 8.0 & 12.5 & 1,400 & 2.50 & 0.55 & 0.06 & 2.56 & 2.27 & 0.28 & 1.56 & 90 \\
\hline & 2/7/2012 & 19.1 & 820 & 8.0 & 18.8 & ${ }^{2} 1,450$ & 3.15 & 0.15 & 0.13 & 2.36 & 2.14 & 0.27 & 1.48 & 112 \\
\hline \multirow[t]{5}{*}{ WD 16} & $10 / 25 / 2008$ & 18.7 & 470 & 8.0 & 7.7 & 780 & - & - & - & - & - & - & - & - \\
\hline & $4 / 27 / 2009$ & 18.7 & 440 & 7.7 & 8.7 & 970 & - & 0.02 & 0.02 & 0.28 & 0.13 & 0.04 & 0.43 & 27.0 \\
\hline & $11 / 24 / 2009$ & 18.7 & 450 & 7.7 & 7.1 & 760 & $<0.66$ & 0.03 & 0.04 & 0.12 & 0.01 & 0.01 & - & 10.6 \\
\hline & $3 / 16 / 2010$ & 18.7 & 440 & 7.6 & 5.1 & 770 & $<0.66$ & 0.03 & 0.02 & 0.13 & 0.04 & 0.01 & - & 5.6 \\
\hline & $3 / 8 / 2011$ & 18.5 & 480 & 7.7 & 4.1 & 770 & $<0.15$ & 0.04 & 0.03 & 0.07 & 0.01 & 0.02 & 0.2 & 21 \\
\hline
\end{tabular}


Table 2. Field water-quality parameters, dissolved organic carbon, tritium, chlorofluorocarbons, sulfur hexafluoride, and neon excess in groundwater and surface water from Sand Hollow, Utah.-Continued

[Analyzing agency: Dissolved organic carbon at U.S. Geological Survey (USGS) N ational Water Quality Laboratory in Denver, Colorado; Tritium at U niversity of U tah. Dissolved G as Laboratory in Salt Lake City, U tah; CFC-11, CFC-12, CFC-113, SF at USGS Chlorofluorocarbon (CFC) Laboratory in Reston, Virginia. Water temperature: ${ }^{\circ} \mathrm{C}$, degrees Celsius; Specific conductance: $\mu \mathrm{S} / \mathrm{cm}$, microsiemens per centimeter at 25 degrees Celsius; Dissolved oxygen, dissolved organic carbon: $\mathrm{mg} / \mathrm{L}$, milligrams per liter; Total dissolved-gas pressure: $\mathrm{mm} \mathrm{Hg}$, millimeters of mercury; Tritium, Tritium precision: TU, tritium units; CFC-11, CFC-12, CFC-113: pmol/kg, picomoles per kilogram; SF $_{6}$ : fmol/kg, femtomoles per kilogram; -, no data available; E, estimated; >, greater than; <, less than]

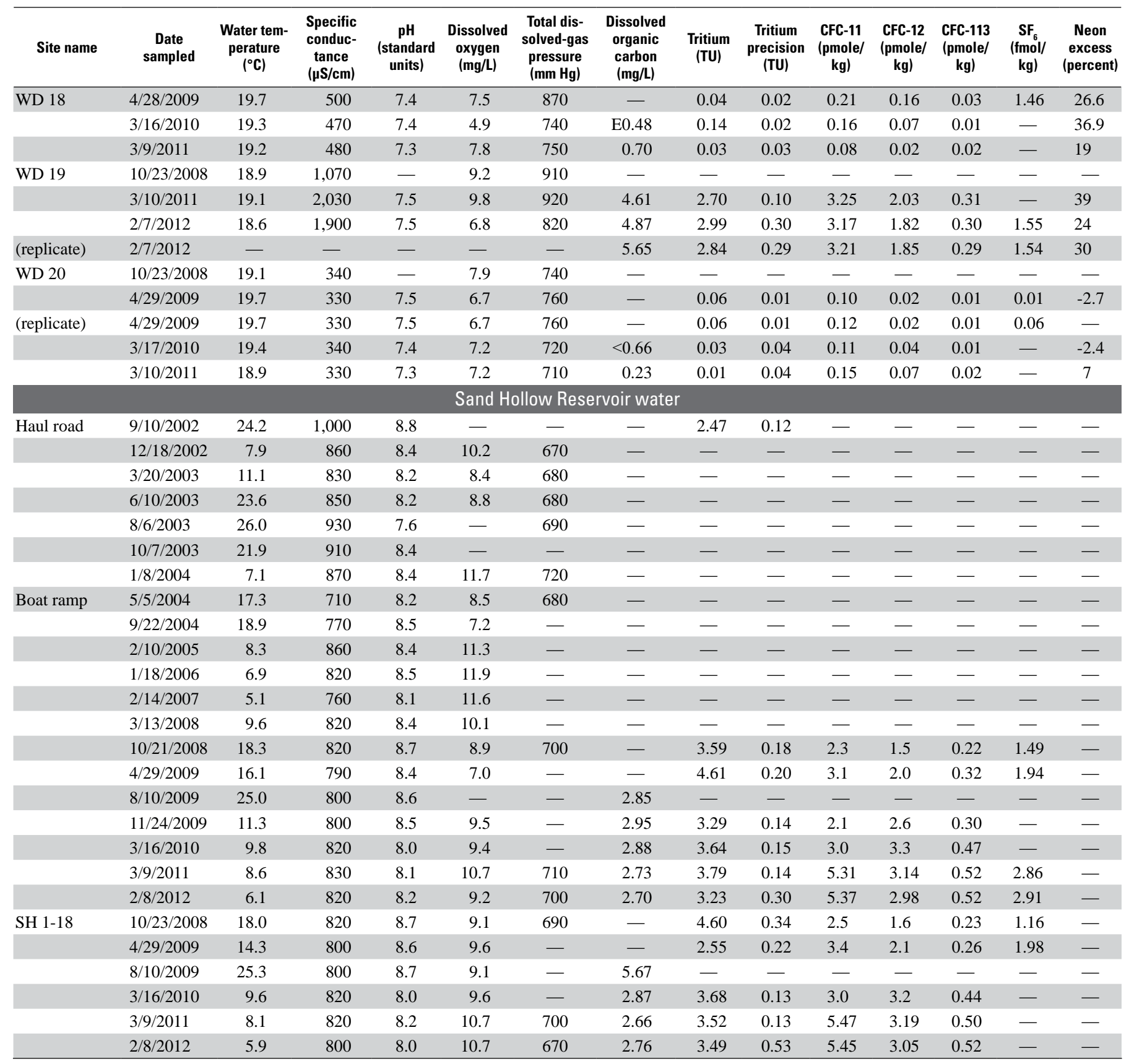

${ }^{1}$ Total dissolved-gas pressures greater than $1,500 \mathrm{~mm}$ Hg exceed the linear calibration of the multi-parameter sonde.

${ }^{2}$ Total dissolved-gas pressure determined with advanced diffusion sampler. 

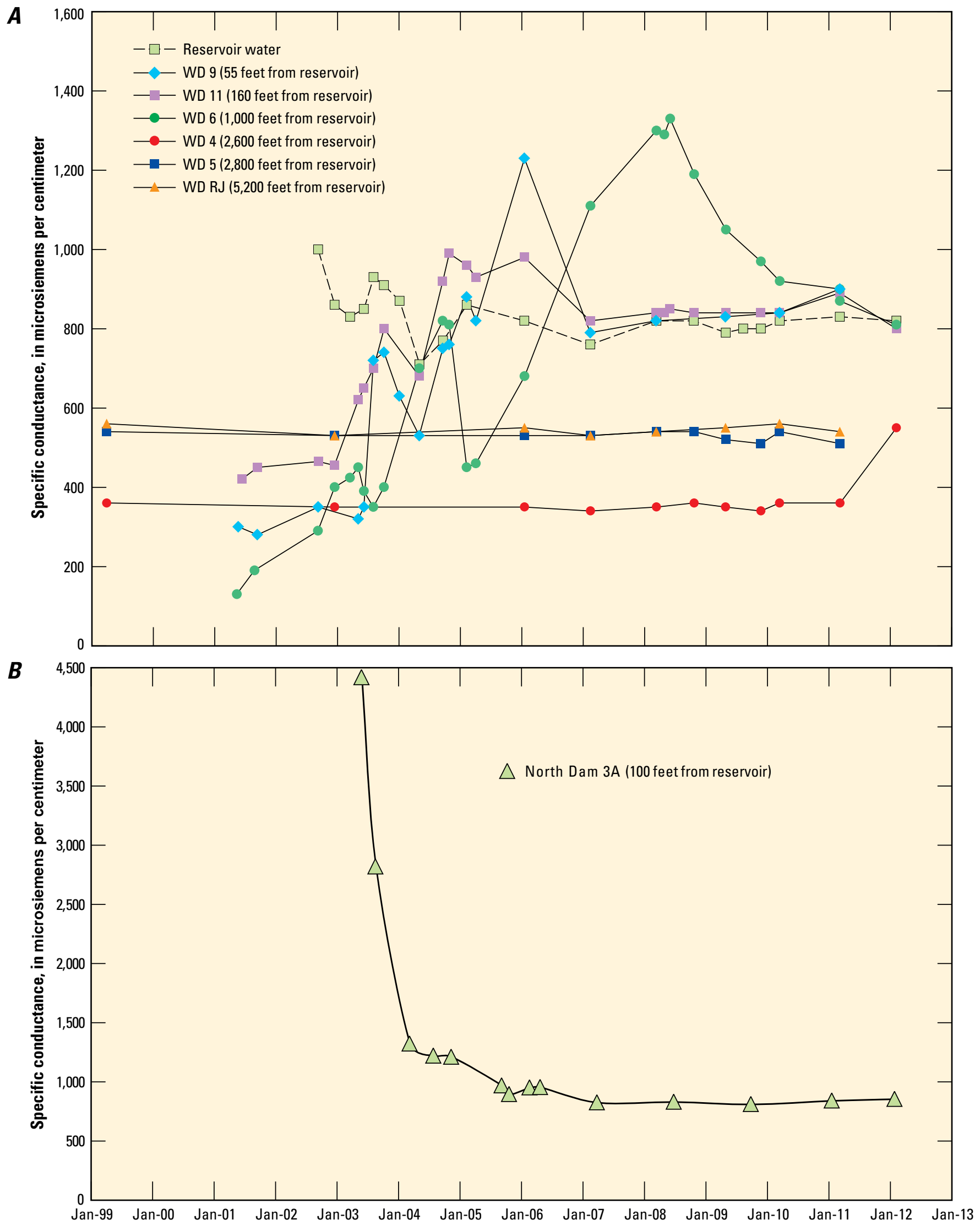

Figure 13. Specific conductance of reservoir water and groundwater from selected monitoring wells in Sand Hollow, Utah, 1999-2012. 
Table 3. Major and minor chemical constituents in groundwater and surface water from selected sites in Sand Hollow, Utah.

[Specific conductance: $\mathrm{mg} / \mathrm{L}$, milligrams per liter; $\mu \mathrm{S} / \mathrm{cm}$, microsiemens per centimeter at 25 degrees Celsius; Temperature: ${ }^{\circ} \mathrm{C}$, degrees $\mathrm{Celsius;} \mathrm{Cl}: \mathrm{Br}$, chloride-to-bromide ratio; $\mu \mathrm{g} / \mathrm{L}$, micrograms per liter; <, less than; E, estimated; —, no data available; ft, feet; repl., replicate]

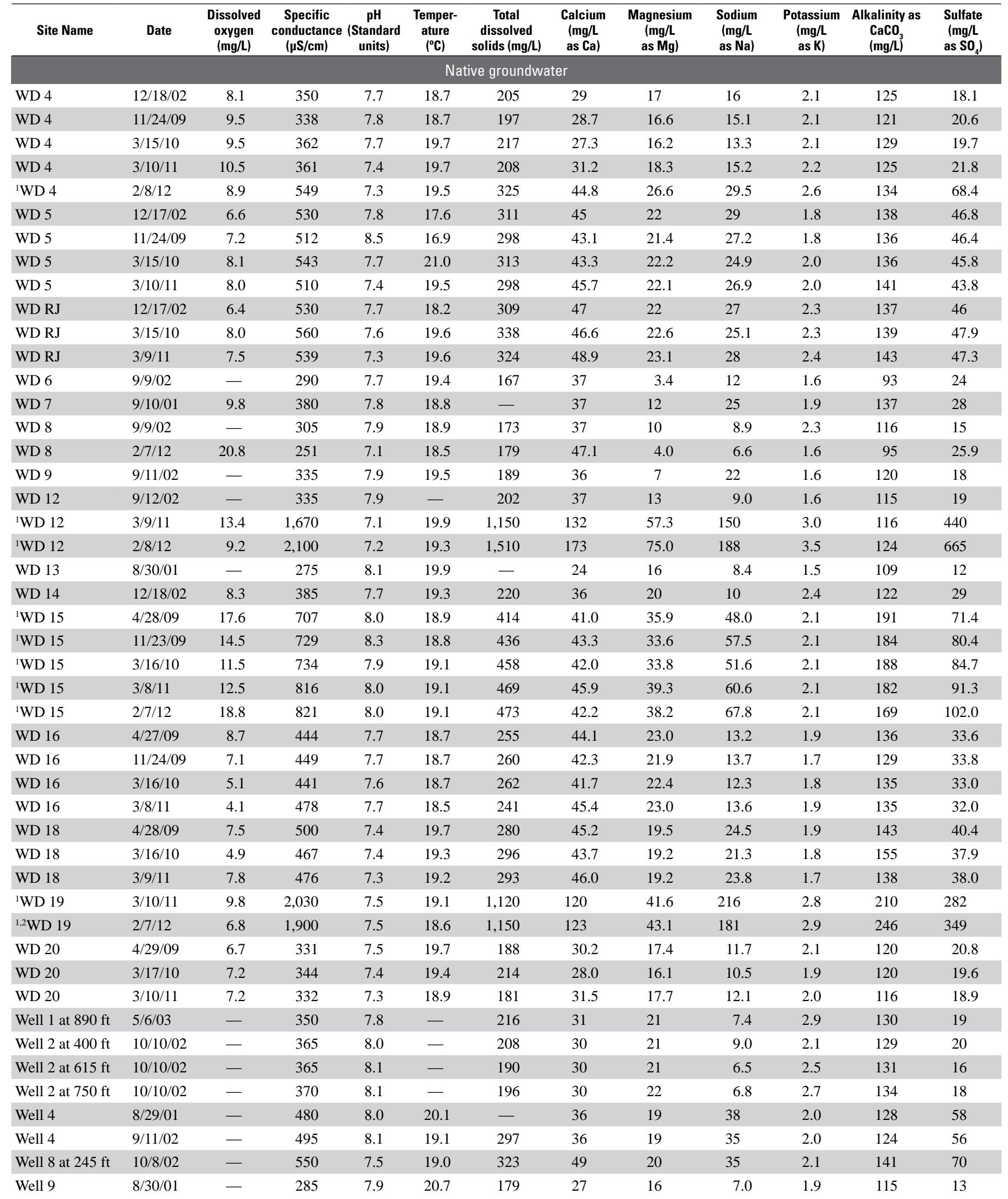


Table 3. Major and minor chemical constituents in groundwater and surface water from selected sites in Sand Hollow, Utah.Continued

[Specific conductance: $\mu \mathrm{S} / \mathrm{cm}$, microsiemens per centimeter at 25 degrees Celcius; $\mathrm{mg} / \mathrm{L}$, milligrams per liter; $\mu \mathrm{g} / \mathrm{L}$, micrograms per liter; Temperature: ${ }^{\circ} \mathrm{C}$, degrees Celcius; - , no data available; ft, feet; $\mathrm{Cl}: \mathrm{Br}$, chloride-to-bromide ratio; repl., replicate; $\mathrm{E}$, estimate; <, less than]

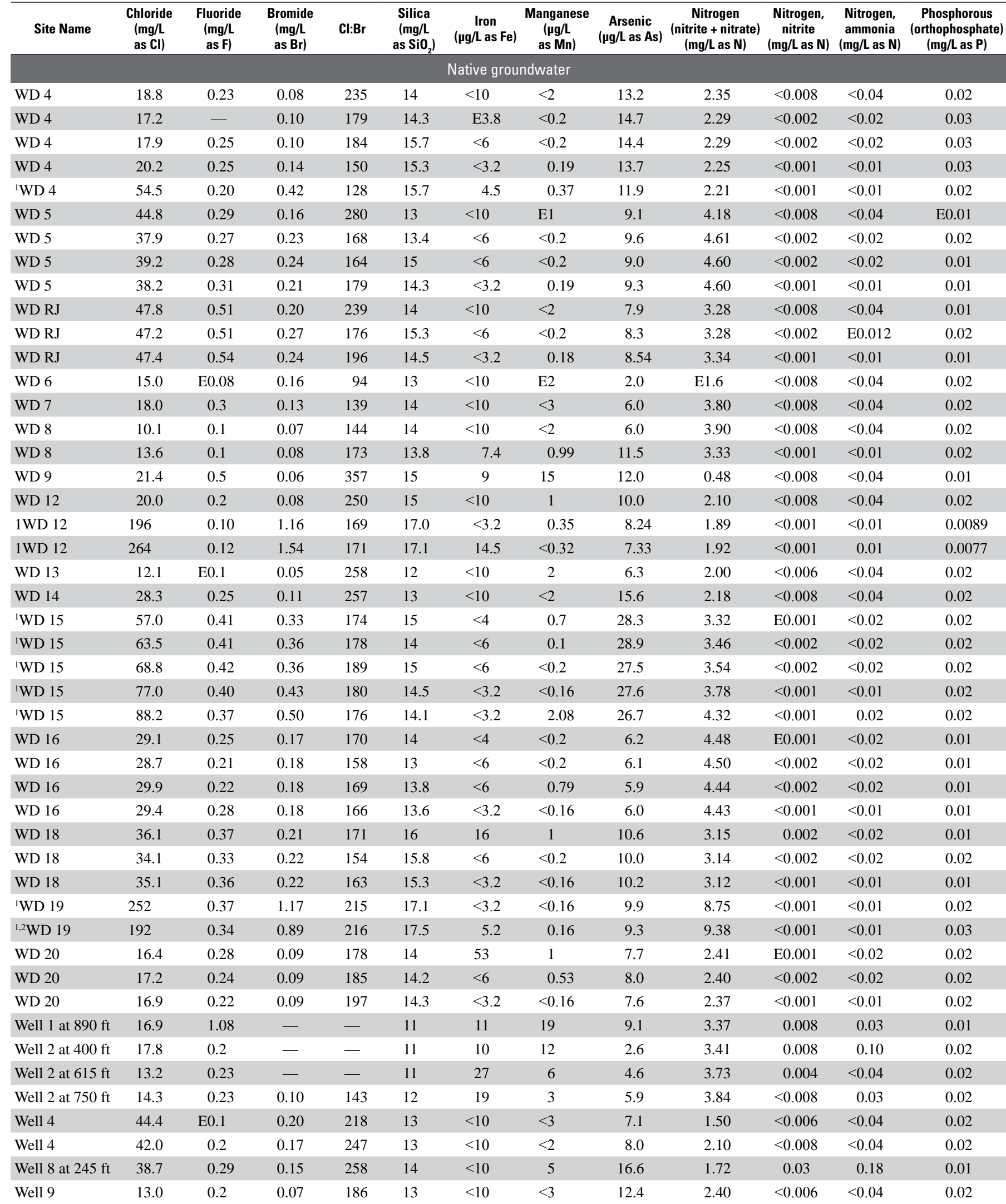


Table 3. Major and minor chemical constituents in groundwater and surface water from selected sites in Sand Hollow, Utah.Continued

[Specific conductance: $\mathrm{mg} / \mathrm{L}$, milligrams per liter; $\mu \mathrm{S} / \mathrm{cm}$, microsiemens per centimeter at 25 degrees Celsius; Temperature: ${ }^{\circ} \mathrm{C}$, degrees $\mathrm{Celsius;} \mathrm{Cl}: \mathrm{Br}$, chloride-to-bromide ratio; $\mu \mathrm{g} / \mathrm{L}$, micrograms per liter; <, less than; E, estimated; —, no data available; ft, feet; repl., replicate]

\begin{tabular}{|c|c|c|c|c|c|c|c|c|c|c|c|c|}
\hline Site Name & Date & $\begin{array}{c}\text { Dissolved } \\
\text { oxygen } \\
\text { (mg/L) }\end{array}$ & $\begin{array}{c}\text { Specific } \\
\text { conductance } \\
(\mu \mathrm{S} / \mathrm{cm})\end{array}$ & $\begin{array}{c}\mathrm{pH} \\
\text { (Standard } \\
\text { units) }\end{array}$ & $\begin{array}{l}\text { Temper- } \\
\text { ature } \\
\left({ }^{\circ} \mathrm{C}\right)\end{array}$ & $\begin{array}{c}\text { Total } \\
\text { dissolved } \\
\text { solids (mg/L) }\end{array}$ & $\begin{array}{c}\text { Calcium } \\
\text { (mg/L } \\
\text { as Ca) }\end{array}$ & $\begin{array}{c}\text { Magnesium } \\
(\mathbf{m g} / \mathbf{L} \\
\text { as } \mathbf{M g})\end{array}$ & $\begin{array}{c}\text { Sodium } \\
(\mathrm{mg} / \mathrm{L} \\
\text { as Na) }\end{array}$ & $\begin{array}{c}\text { Potassium } \\
\text { (mg/L } \\
\text { as K) }\end{array}$ & $\begin{array}{c}\text { Alkalinity as } \\
\mathrm{CaCO}_{3} \\
(\mathrm{mg} / \mathrm{L})\end{array}$ & $\begin{array}{c}\text { Sulfate } \\
(\mathrm{mg} / \mathrm{L} \\
\left.\text { as SO})_{4}\right)\end{array}$ \\
\hline \multicolumn{13}{|c|}{ Groundwater affected by reservoir recharge } \\
\hline North Dam $3 A$ & $12 / 18 / 02$ & 10.8 & 2,830 & 8.0 & 14.7 & 1,890 & 110 & 110 & 340 & 3.6 & 155 & 584 \\
\hline North Dam $3 A$ & $3 / 16 / 10$ & 1.3 & 864 & 7.6 & 22.8 & 554 & 65.8 & 38.0 & 51.2 & 3.0 & 177 & 187 \\
\hline WD 6 & $4 / 30 / 09$ & 22.0 & 1,045 & 7.7 & 19.2 & 660 & 98.5 & 9.0 & 113 & 1.6 & 169 & 220 \\
\hline WD 6 & $11 / 23 / 09$ & 15.3 & 968 & 7.9 & 18.9 & 629 & 93.6 & 8.7 & 101 & 1.5 & 161 & 210 \\
\hline WD 6 & $3 / 15 / 10$ & 14.4 & 923 & 7.5 & 19.2 & 618 & 94.1 & 8.6 & 86.3 & 1.5 & 166 & 211 \\
\hline WD 6 & $3 / 9 / 11$ & 11.2 & 896 & 7.5 & 19.1 & 577 & 106 & 11.5 & 73.4 & 1.49 & 157 & 208 \\
\hline WD 9 & $3 / 15 / 10$ & 1.7 & 842 & 7.3 & 16.4 & 543 & 71.8 & 31.0 & 52.2 & 3.3 & 157 & 200 \\
\hline WD 9 - repl. & $3 / 15 / 10$ & 1.7 & 842 & 7.3 & 16.4 & 545 & 67.4 & 28.8 & 51.0 & 3.2 & 155 & 198 \\
\hline WD 9 & $3 / 8 / 11$ & 0.5 & 902 & 7.3 & 16.1 & 531 & 73.8 & 33.9 & 60.9 & 3.58 & 161 & 188 \\
\hline WD 9 & $2 / 7 / 12$ & 0.4 & 826 & 7.1 & 16.3 & 533 & 66.4 & 34.2 & 56.6 & 3.45 & 162 & 185 \\
\hline WD 11 & $5 / 3 / 04$ & 21.5 & 677 & 7.7 & 18.4 & 440 & 69.0 & 31.6 & 68.1 & 1.7 & 187 & 90 \\
\hline WD 11 & $4 / 30 / 09$ & 19.4 & 843 & 7.7 & 15.9 & 557 & 79.2 & 38.6 & 49.6 & 2.4 & 186 & 187 \\
\hline WD 11 & $11 / 23 / 09$ & 13.2 & 835 & 7.9 & 16.3 & 553 & 74.0 & 35.7 & 49.4 & 2.2 & 171 & 191 \\
\hline WD 11 & $3 / 15 / 10$ & 10.3 & 837 & 7.7 & 16.2 & 552 & 67.2 & 34.3 & 45.6 & 2.2 & 178 & 190 \\
\hline WD 11 & $3 / 8 / 11$ & 9.9 & 891 & 7.7 & 16.0 & 533 & 75.2 & 36.6 & 51.2 & 2.15 & 186 & 179 \\
\hline WD 11 & $2 / 7 / 12$ & 9.5 & 798 & 7.4 & 15.4 & 529 & 74.6 & 34.5 & 51.6 & 2.05 & 193 & 169 \\
\hline B oat ramp & $11 / 24 / 09$ & 9.5 & 797 & 8.5 & 11.3 & 502 & 40.9 & 39.8 & 62.9 & 4.3 & 108 & 212 \\
\hline B oat ramp & $3 / 16 / 10$ & 9.4 & 817 & 8.0 & 9.8 & 534 & 43.5 & 38.4 & 57.6 & 4.6 & 120 & 211 \\
\hline B oat ramp & $3 / 9 / 11$ & 10.7 & 827 & 8.1 & 8.6 & 534 & 60.2 & 39.2 & 62.2 & 4.5 & 142 & 212 \\
\hline B oat ramp & $2 / 6 / 12$ & 9.2 & 821 & 8.2 & 6.1 & 534 & 53.6 & 39.8 & 61.3 & 4.3 & 138 & 213 \\
\hline SH 1-18 & $4 / 29 / 09$ & 9.6 & 800 & 8.6 & 14.3 & 502 & 56.1 & 37.2 & 53.6 & 4.2 & 146 & 190 \\
\hline SH 1-18 & $8 / 10 / 09$ & 9.1 & 800 & 8.7 & 25.3 & 501 & 42.6 & 38.3 & 60.5 & 4.3 & 110 & - \\
\hline SH 1-18 & $3 / 16 / 10$ & 9.6 & 819 & 8.0 & 9.6 & 525 & 45.9 & 40.8 & 58.6 & 4.7 & 124 & 211 \\
\hline SH 1-18 & $3 / 9 / 11$ & 10.7 & 820 & 8.2 & 8.1 & 528 & 60.1 & 39.6 & 61.2 & 4.43 & 140 & 210 \\
\hline SH 1-18 & $2 / 8 / 12$ & 10.7 & 801 & 8.0 & 5.9 & 526 & 54.2 & 39.5 & 59.6 & 4.28 & 140 & 214 \\
\hline
\end{tabular}

${ }^{1}$ High or increasing total dissolved solids but low $\mathrm{Cl}: \mathrm{Br}$ ratio indicate groundwater is affected by flushing of naturally occuring solute accumulations in vadose zone prior to reservoir construction.

${ }^{2}$ Replicate sample not reported because submersible pump was re-installed for replicate collection and total dissolved solids differed by more than 10 percent. 
Table 3. Major and minor chemical constituents in groundwater and surface water from selected sites in Sand Hollow, Utah.Continued

[Specific conductance: $\mu \mathrm{S} / \mathrm{cm}$, microsiemens per centimeter at 25 degrees Celcius; $\mathrm{mg} / \mathrm{L}$, milligrams per liter; $\mu \mathrm{g} / \mathrm{L}$, micrograms per liter; Temperature: ${ }^{\circ} \mathrm{C}$, degrees Celcius; - , no data available; ft, feet; $\mathrm{Cl}: \mathrm{Br}$, chloride-to-bromide ratio; repl., replicate; E, estimate; <, less than]

\begin{tabular}{|c|c|c|c|c|c|c|c|c|c|c|c|c|}
\hline Site Name & $\begin{array}{l}\text { Chloride } \\
\text { (mg/L } \\
\text { as Cl) }\end{array}$ & $\begin{array}{c}\text { Fluoride } \\
\text { (mg/L } \\
\text { as F) }\end{array}$ & $\begin{array}{c}\text { Bromide } \\
\text { (mg/L } \\
\text { as Br) }\end{array}$ & $\mathrm{Cl}: \mathrm{Br}$ & $\begin{array}{c}\text { Silica } \\
(\mathrm{mg} / \mathrm{L} \\
\left.\text { as } \mathrm{SiO}_{2}\right)\end{array}$ & $\underset{\text { Iron }}{\operatorname{lgg} / \mathrm{L} \text { as } \mathrm{Fe})}$ & $\begin{array}{c}\text { Manganese } \\
(\mu \mathrm{g} / \mathrm{L} \\
\text { as } \mathrm{Mn})\end{array}$ & $\begin{array}{c}\text { Arsenic } \\
\text { ( } \mu \mathrm{g} / \mathrm{L} \text { as As) }\end{array}$ & $\begin{array}{c}\text { Nitrogen } \\
\text { (nitrite + nitrate) } \\
\text { (mg/L as N) }\end{array}$ & $\begin{array}{c}\text { Nitrogen, } \\
\text { nitrite } \\
\text { (mg/L as N) }\end{array}$ & $\begin{array}{c}\text { Nitrogen, } \\
\text { ammonia } \\
\text { (mg/L as N) }\end{array}$ & $\begin{array}{c}\text { Phosphorous } \\
\text { (orthophosphate) } \\
\text { (mg/L as P) }\end{array}$ \\
\hline \multicolumn{13}{|c|}{ Groundwater affected by reservoir recharge } \\
\hline North Dam $3 \mathrm{~A}$ & 476 & 0.79 & 2.44 & 195 & 14 & $<30$ & $<5$ & 63.9 & 14.3 & $<0.008$ & $<0.04$ & 0.03 \\
\hline North Dam $3 \mathrm{~A}$ & 55.2 & 0.43 & 0.06 & 882 & 16 & $<6$ & 3.19 & 35.2 & $<0.04$ & E0.001 & E0.01 & 0.03 \\
\hline WD 6 & 92.5 & 0.32 & 0.31 & 295 & 13 & $<4$ & 0.23 & 3.3 & 1.2 & $<0.002$ & $<0.02$ & 0.01 \\
\hline WD 6 & 80.3 & 0.30 & 0.28 & 286 & 12 & $<6$ & $<0.2$ & 3.3 & 1.06 & $<0.002$ & $<0.02$ & 0.01 \\
\hline WD 6 & 77.9 & 0.32 & 0.24 & 322 & 13 & 20.8 & 0.32 & 3.0 & 0.97 & $<0.002$ & $<0.02$ & 0.01 \\
\hline WD 6 & 63.5 & 0.30 & 0.14 & 457 & 13.5 & 6.3 & 0.4 & 2.82 & 0.71 & $<0.001$ & $<0.01$ & 0.01 \\
\hline WD 9 - repl. & 56.3 & 0.27 & 0.05 & 1,083 & 12 & 13.5 & 0.57 & 6.1 & 0.09 & $<0.002$ & $<0.02$ & 0.01 \\
\hline WD 9 & 58.7 & 0.28 & 0.04 & 1,310 & 11.6 & 6.7 & 0.47 & 6.14 & 0.05 & 0.002 & $<0.01$ & 0.01 \\
\hline WD 9 & 55.6 & 0.27 & 0.06 & 927 & 11.3 & 26.3 & 2.53 & 5.87 & 0.06 & $<0.001$ & 0.01 & 0.01 \\
\hline WD 11 & 49.8 & 0.40 & 0.25 & 199 & 15 & $<6$ & $<0.8$ & 15.3 & 3.06 & $<0.008$ & $<0.04$ & 0.02 \\
\hline WD 11 & 49.6 & 0.35 & 0.07 & 687 & 14 & $<4$ & $<0.2$ & 9.6 & 0.99 & $<0.002$ & $<0.02$ & 0.01 \\
\hline WD 11 & 49.8 & 0.31 & 0.07 & 711 & 13 & $<6$ & $<0.2$ & 10.3 & 0.67 & $<0.002$ & $<0.02$ & 0.01 \\
\hline WD 11 & 51.8 & 0.32 & 0.07 & 781 & 14 & 18.6 & 0.26 & 10.0 & 0.70 & $<0.002$ & $<0.02$ & 0.02 \\
\hline WD 11 & 53.6 & 0.31 & 0.07 & 811 & 14.7 & 4.4 & 0.26 & 9.57 & 0.48 & 0.001 & $<0.01$ & 0.01 \\
\hline WD 11 & 53.3 & 0.26 & 0.07 & 733 & 15.3 & 11.4 & 0.34 & 9.16 & 0.48 & $<0.001$ & $<0.01$ & 0.01 \\
\hline B oat ramp & 60.4 & 0.28 & 0.05 & 1,313 & 1.5 & $<6$ & 0.2 & 1.6 & $<0.04$ & $<0.002$ & $<0.02$ & 0.008 \\
\hline B oat ramp & 61.7 & 0.30 & 0.04 & 1,374 & 1.4 & 6.3 & 1.7 & 1.3 & E0.033 & $<0.002$ & 0.02 & $<0.008$ \\
\hline B oat ramp & 57.0 & 0.30 & 0.04 & 1,390 & 4.0 & $<3.2$ & 2.0 & 1.13 & 0.10 & 0.004 & 0.03 & $<0.004$ \\
\hline B oat ramp & 58.3 & 0.27 & 0.05 & 1,108 & 4.2 & $<3.2$ & 0.86 & 1.01 & 0.12 & 0.001 & 0.01 & $<0.004$ \\
\hline SH 1-18 & 54.6 & 0.27 & 0.04 & 1,318 & 3.0 & $<4$ & 0.4 & 1.4 & 0.04 & 0.003 & 0.13 & 0.008 \\
\hline SH 1-18 & - & 0.24 & - & - & 1.3 & 3 & 0.3 & 1.6 & $<0.04$ & $<0.002$ & $<0.02$ & 0.008 \\
\hline SH 1-18 & 61.6 & 0.30 & 0.04 & 1,417 & 1.2 & 6.2 & 1.8 & 1.4 & E0.025 & $<0.002$ & 0.02 & $<0.008$ \\
\hline SH 1-18 & 57.2 & 0.30 & 0.04 & 1,546 & 3.9 & $<3.2$ & 2.10 & 1.12 & 0.10 & 0.005 & 0.02 & $<0.004$ \\
\hline SH 1-18 & 56.5 & 0.27 & 0.05 & 1,060 & 4.1 & $<3.2$ & 1.31 & 1.04 & 0.09 & 0.001 & 0.02 & $<0.004$ \\
\hline
\end{tabular}

\footnotetext{
${ }^{1}$ High or increasing total dissolved solids but low $\mathrm{Cl}: \mathrm{Br}$ ratioindicate groundwater is affected by flushing of naturally occuring solute accumulations in vadose zone prior to reservoir construction.

${ }^{2}$ Replicate sample not reported because submersible pump was re-installed for replicate collection and total dissolved solids differed by more than 10 percent.
} 


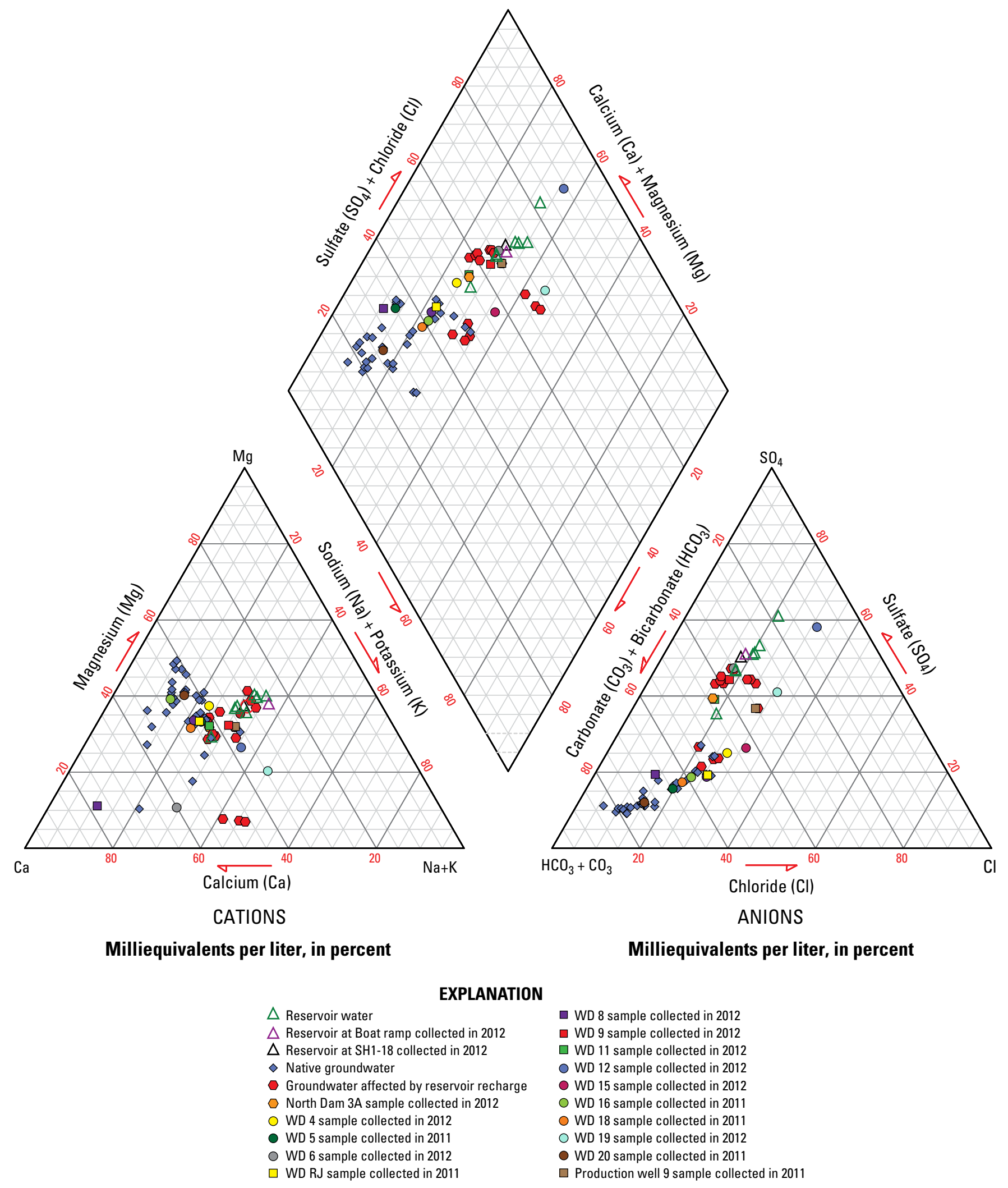

Figure 14. Major-ion chemistry from selected surface water and groundwater sites in Sand Hollow, Utah. 
travel times of less than 1 year for reservoir recharge to reach North Dam $3 A$ and about $2.5,4$, and 6 years to reach WD 11 , WD 9, and WD 6, respectively. By 2012, specific conductance at all four of these monitoring wells declined to values measured in the reservoir (about $800 \mu \mathrm{S} / \mathrm{cm}$ ). The specific conductance at W D 4 began rising slightly in 2012, possibly indicating the arrival of reservoir water in the near future. While specific conductance was also elevated at monitoring wells WD 12, WD 15, and WD 19, low chloride-to-bromide ratios $(\mathrm{Cl}: \mathrm{Br}$, discussed below) indicate that these increases could be caused by mobilization of natural vadose-zone salts near the well screen with rising water levels (discussed in Heilw eil and $M$ arston, 2011) rather than marking the arrival of reservoir water. The other monitoring wells (WD 5, WD RJ, WD 8, WD 16 , WD 18, WD 20) showed no substantial increase in specific conductance through 2012.

\section{Laboratory Water Chemistry}

\section{Dissolved Inorganic lons}

Major-ion chemical signatures can be used to identify the arrival of reservoir recharge from Sand Hollow Reservoir at downgradient monitoring wells. Figure 14 shows that groundwater at some sites in Sand Hollow has been affected by recharge from the reservoir, while other sites have not. The trilinear (Piper) diagram shows that native (background) groundwater in Sand Hollow is lower in chloride, magnesium, and sodium (plus potassium) and higher in calcium and bicarbonate than the infiltrating reservoir water. Groundwater influenced by reservoir recharge typically plots between native groundwater and reservoir water, indicating that groundwater at these sites has shifted from calcium bicarbonate- to sodium chloride-type water. Exceptions to this are seen in samples collected from WD 12 and WD 19, where a geochemical signature different than reservoir water could reflect the mobilization of vadose-zone salts.

Chloride-to-bromide ratios $(\mathrm{Cl}: \mathrm{Br})$ of water from Sand Hollow Reservoir are useful for tracing the movement of recharge from the reservoir through the aquifer. $\mathrm{Cl}: \mathrm{Br}$ ratios in the reservoir fluctuated between 1,100 and 5,000 from 2003 through 2006 because of leaching of surficial salts while the reservoir was being initially filled (fig. 15). Beginning in 2007, the ratios in Sand Hollow Reservoir gradually increased from about 1,000 to 1,400, then in 2012, declined to about 1,100. In contrast, $\mathrm{Cl}: \mathrm{Br}$ ratios of native groundwater in Sand $\mathrm{H}$ ollow are much lower at about 90 to 280 (table 3). $\mathrm{Cl}: \mathrm{Br}$ ratios in wells receiving recharge from the reservoir ( $N$ orth $D$ am $3 A$, WD 6. WD 9, WD 11) generally rose in recent years, indicating the arrival of reservoir recharge. $\mathrm{Cl}: \mathrm{Br}$ ratios did not rise at four other monitoring wells (WD 4, WD 12, WD 15, WD 19) showing recent increases in specific conductance and chloride concentration, indicating a mobilization of natural vadosezone salts rather than the arrival of reservoir water.

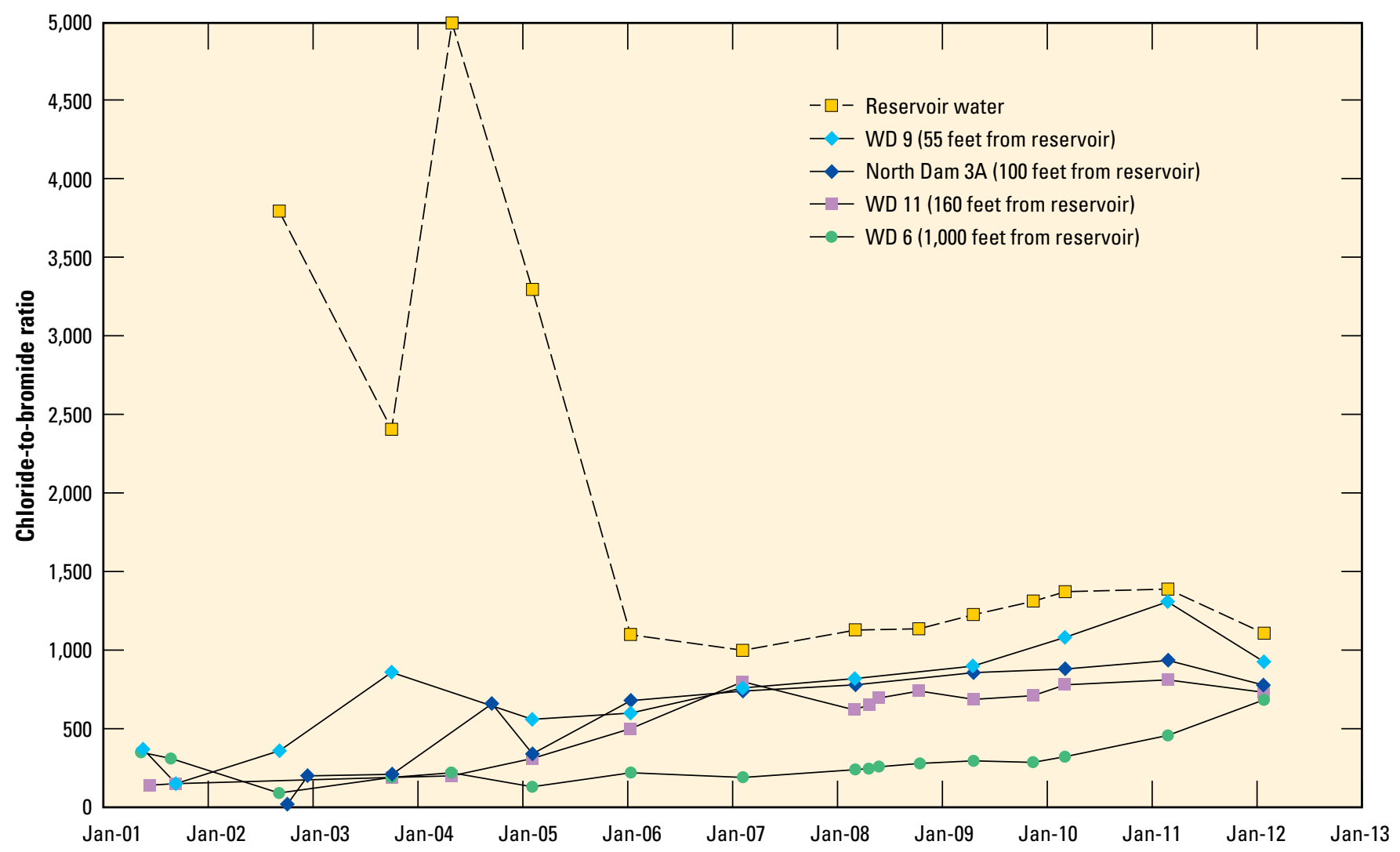

Figure 15. Chloride-to-bromide ratios of reservoir water and groundwater from selected monitoring wells in Sand Hollow, Utah, $2001-12$. 


\section{Dissolved Organic Carbon, Tritium, and Dissolved Gases}

Dissolved organic carbon (DOC) is another potential tracer of reservoir recharge, and it has been included in Sand $\mathrm{Hol}$ low water-quality sampling since 2008. M easured values of DOC in Sand Hollow Reservoir water ranged from 2.7 to 5.7 $\mathrm{mg} / \mathrm{L}$ (table 2 ). In contrast, native groundwater at monitoring wells not yet affected by reservoir recharge (WD 5, WD RJ, WD 16, WD 18, WD 20) generally had relatively low DOC concentrations of 0.4 to $0.8 \mathrm{mg} / \mathrm{L}$. Groundwater at monitoring wells North Dam 3A, WD 6, WD 9, and WD 11, all affected by reservoir recharge based on elevated $\mathrm{Cl}: \mathrm{Br}$ ratios, had DOC concentrations of 1.2 to $2.0 \mathrm{mg} / \mathrm{L}$. Elevated DOC $(0.8$ to $5.7 \mathrm{mg} / \mathrm{L}$ ) also was measured at wells WD 4, WD 8, WD 12 , WD 15, and WD 19, where rising or elevated concentrations of other parameters (specific conductance, tritium, CFCs, $\mathrm{SF}_{6}$ ) but low $\mathrm{Cl}: \mathrm{Br}$ ratios, could indicate the mobilization of vadose-zone salts or local natural recharge rather than arrival of reservoir water. A lthough DOC could be an indicator of the arrival of reservoir recharge, further data collection is needed to evaluate other potential sources of DOC in groundwater within Sand Hollow.

Tritium concentrations in reservoir water and groundwater have been measured sporadically since 1999. Like specific conductance, TDG pressure and dissolved industrial gases, surface-water tritium concentrations were generally higher than in groundwater, making it a useful tracer of reservoir recharge. Reservoir water has had tritium concentrations of 2.5 to 4.6 TU, with values generally stable at about 3.5 TU since 2009 (table 2). In contrast, native groundwater in monitoring wells, sampled either before the reservoir was constructed or those sampled more recently but away from the reservoir, has tritium concentrations generally less than about $0.5 \mathrm{TU}$ (fig. 16), indicating that the majority of natural recharge infiltrated prior to the $1960 \mathrm{~s}$. The exception to this is WD 8 , which had 3 to 4 TU prior to construction of the reservoir, indicating higher natural recharge rates through infiltration of precipitation. The monitoring wells al ready receiving reservoir recharge (N orth Dam 3A, WD 6, WD 9, WD 11) have had tritium concentrations of 2.1 to $3.6 \mathrm{TU}$ since 2008. Because of low $\mathrm{Cl}: \mathrm{Br}$ ratios (less than 250 ), the el evated tritium concentrations at WD 12 and WD 19 (about 1 to 3 TU) could indicate the arrival of modern natural recharge rather than reservoir recharge.

Chlorofluorocarbons (CFC-11, CFC-12, and CFC-113) and sulfur hexafluoride $\left(\mathrm{SF}_{6}\right)$ are trace atmospheric gases that have been sampled in Sand Hollow since 2008, as additional indicators of reservoir recharge. CFC-12 is considered the most stable of the three chlorofluorocarbons-both CFC-11 and CFC -113 are more likely to be affected by microbial decay. Dissolved CFC-12 concentrations in surface water collected from Sand Hollow Reservoir gradually increased from about 1.5 to $3.0 \mathrm{pmol} / \mathrm{kg}$ between 2008 and 2010 (H eilweil and $M$ arston, 2011), then remained relatively stable during 2011-2012 (table 2; fig. 17). Monitoring wells sampled near the reservoir during 2011-2012 (N orth Dam 3A, WD 6, WD
9, WD 11) had CFC - 12 concentrations ranging from 1.7 to $2.6 \mathrm{pmol} / \mathrm{kg}$, signifying the arrival of reservoir recharge. Low CFC -12 concentrations (less than $0.2 \mathrm{pmol} / \mathrm{kg}$ ) measured in 2010 and 2011 at monitoring wells farther from the reservoir (WD 5, WD RJ, WD 16, WD 18, WD 20), indicate older native groundwater. D uring 2011-2012, higher CFC-12 concentrations (1.7 to $2.2 \mathrm{pmol} / \mathrm{kg}$ ) were measured in the group of wells (WD 8, WD 12, WD 15, WD 19) having elevated tritium concentrations and low $\mathrm{Cl}: \mathrm{Br}$ ratios, which are attributed to modern natural recharge, as discussed previously. The unique locations on the trilinear diagram (fig. 14) of samples from this group of wells is not easily explained. While it is conceivable that reservoir water has reached WD 8 and WD 12 (both located within $1,000 \mathrm{ft}$ of the reservoir), it is unlikely that this has occurred at WD 15 and WD 19, located 2,400 to 5,000 ft from the reservoir and co-located with the deeper (WD 16, WD 20) monitoring wells showing no signs of the arrival of reservoir recharge. Rising specific-conductance values at all wells but W $D 8$ could instead be indicative of the mobilization of nearby vadose-zone salts associated with the rising regional water table.

The measured $\mathrm{SF}_{6}$ concentrations in surface water from the reservoir boat ramp, representing water equilibrated with modern atmospheric concentrations of the gas, increased from about 1.5 to $1.9 \mathrm{fmol} / \mathrm{kg}$ in 2008-2009 to about $2.9 \mathrm{fmol} / \mathrm{kg}$ in 2011-2012 (table 2). This is at least partly attributed to the increase in atmospheric $\mathrm{SF}_{6}$ concentrations of about 7 percent per year, or from about 6.5 to 7.8 pptv from 2008 to 2012 (http://water.usgs.gov/lab/sf6/background/). D uring 20112012, groundwater $\mathrm{SF}_{6}$ concentrations ranged from about 0.1 to $1.5 \mathrm{fmol} / \mathrm{kg}$. The locations of low and high groundwater $\mathrm{SF}_{6}$ concentrations were generally similar to the locations of CFC -12 concentrations. Like CFC- $12, \mathrm{SF}_{6}$ concentrations increased at WD 4 and WD 6 from 2008 to 2012. The increase at WD 6 was due to the increasing fraction of reservoir water at this location, consistent with increasing $\mathrm{Cl}: \mathrm{Br}$ ratios and major-ion geochemical evolution (see fig. 14B of Heilweil and $M$ arston, 2011). WD 4 is unique among all the monitoring wells, showing an increase in dissolved-gas concentrations (both CFCs and SF $_{6}$ ) during 2011-2012, but very low tritium concentrations (less than about $0.1 \mathrm{TU}$ ). Specific conductance of water from this well has recently increased to $550 \mu \mathrm{S} / \mathrm{cm}$ and its geochemical signature is evolving toward that of other groundwater sites affected by reservoir recharge. B ecause it is located about 2,600 ft from the reservoir (similar to WD 15 and WD 5, which are not yet receiving reservoir recharge), this monitoring well could be affected by a combination of the mobilization of vadose-zone salts and recent water-table drawdown (fig. 5) associated with pumping at nearby Well 19. Screened about 80 to $90 \mathrm{ft}$ bel ow land surface, water levels in WD 4 rose from about $20 \mathrm{ft}$ above the screened interval in 2002 to about $50 \mathrm{ft}$ above the screened interval in 2010, likely mobilizing vadose-zone salts. Subsequent, pumping at Well 19 lowered the water level in WD 4 by about $20 \mathrm{ft}$ by 2012, likely bringing younger, higher salinity water toward 


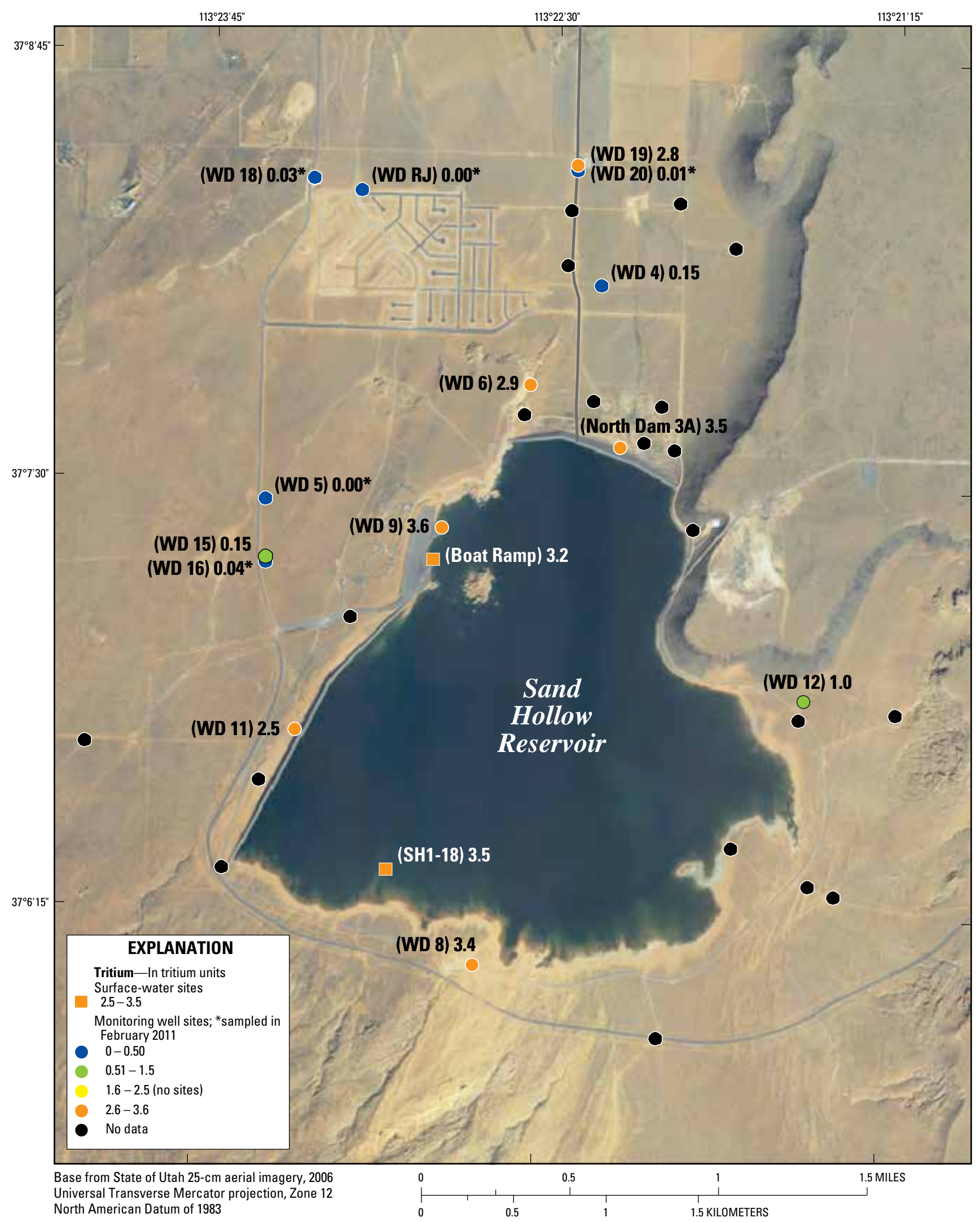

Figure 16. Tritium concentrations of reservoir water and groundwater from selected monitoring wells in Sand Hollow, Utah, February 2012. 


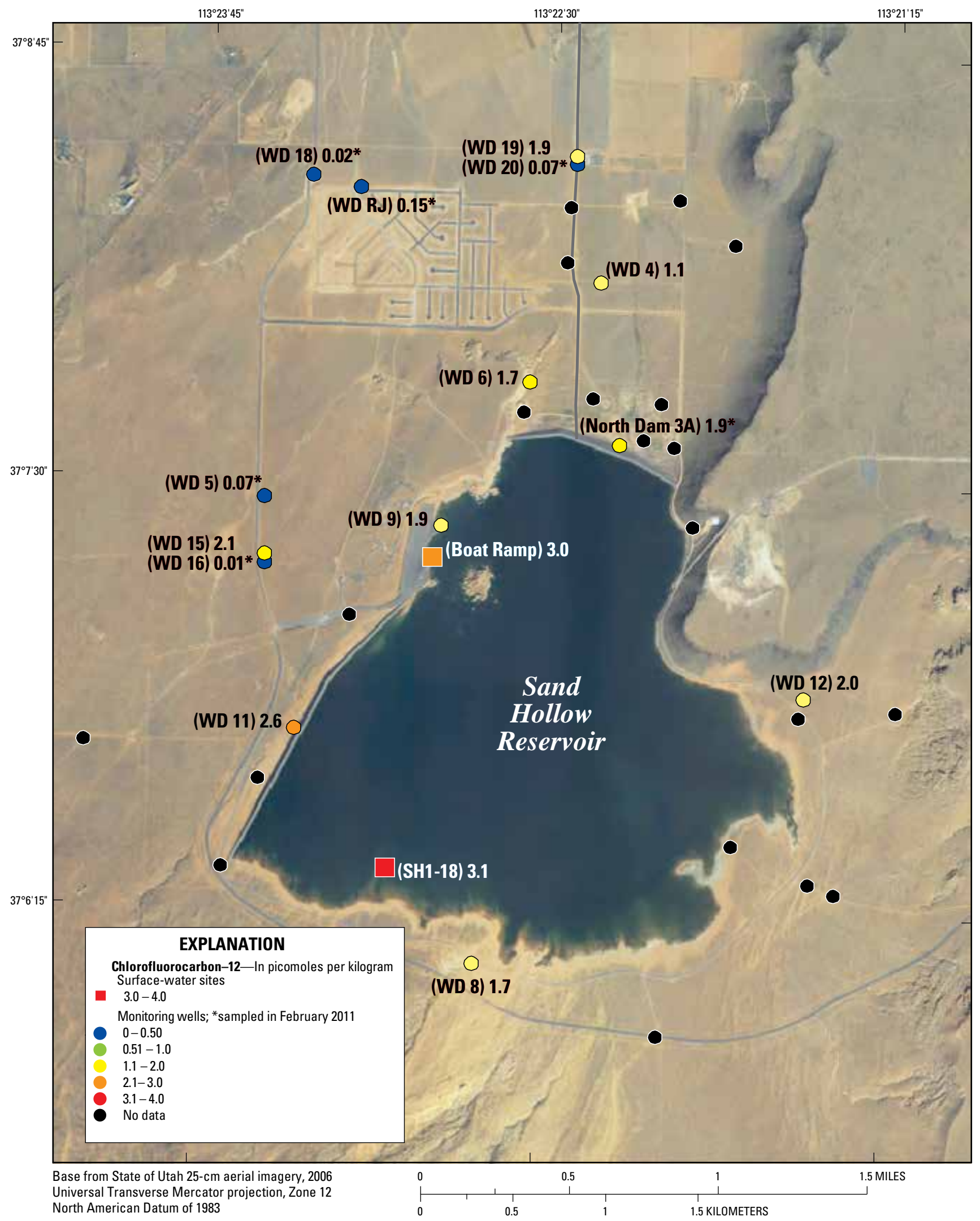

Figure 17. CFC-12 concentrations of reservoir water and groundwater from selected monitoring wells in Sand Hollow, Utah, February 2012. 
the screened interval. While this pumping could explain the changing salinity and ages, the contradiction between modern dissolved gases and pre-modern tritium concentrations at this site requires further investigation.

As discussed in Heilweil and M arston (2011), water samples collected at selected monitoring wells were also analyzed for dissolved noble gases (helium, neon, argon, krypton, and xenon) to allow for the calculation of excess neon by using the closed system equilibrium (CE) model (A eschbach-H ertig and others, 2000). Neon excess refers to the amount of dissolved neon in water above atmospherically equilibrated quantities. High neon-excess values in Sand Hollow indicate dissolution of air bubbles, either associated with the rapidly rising water table or air trapped directly beneath the reservoir as this region became saturated. Prior to the filling of Sand Hollow Reservoir, noble-gas measurements in monitoring wells indicated excess neon concentrations up to about 70 percent (table 2). In contrast, excess-neon values ranged from about 160 to 340 percent in monitoring wells close to the reservoir. While neon excess in most monitoring wells near the reservoir has been increasing, values at WD 9 have rapidly decreased from about 250 percent in 2005 to 30 percent in 2012, indicating the dissolution of most of the initially trapped air beneath the reservoir.

\section{Evaluating the Arrival of Managed Aquifer Recharge at Monitoring Wells}

As described by Heilweil and M arston (2011), changes in values for selected field parameters (TDG pressure, DO, and specific conductance), dissolved chemical constituents $(\mathrm{Cl}: \mathrm{Br}$ ratios, major-ion chemistry, organic carbon), tritium, and dissolved industrial and noble gases (CFC-12, $\mathrm{SF}_{6}$, neon) at the monitoring wells were used to evaluate the movement of managed aquifer recharge through the Navajo Sandstone aquifer in Sand Hollow. While the various tracers show ed recharge from Sand Hollow Reservoir had arrived at several monitoring wells close to the reservoir, they often indicated different peak arrival years for the same monitoring well (table 4). This is likely due to different behavioral characteristics of each of the tracers, such as adsorption and retardation, dispersion, and gas dissolution as recharge enters and moves through the aquifer. In general, these tracers indicated that recharge from the reservoir arrived earliest at the wells located closest to the reservoir (WD 9, North Dam 3A). North Dam 3A was initially a dry borehole in the vadose zone and received recharge from the reservoir in mid-2002, when it first became saturated, even though the peak $\mathrm{Cl}: \mathrm{Br}$ ratio and $\mathrm{DO}$ concentration were not measured until 2004 and 2005, respectively. The recharge arrival year at WD 9 is less certain, with tracer peaks between 2003 and 2011. A tWD 11, most of the tracers indicated that the peak breakthrough of reservoir recharge was likely in 2005 or 2006; the peak in neon excess could have been in 2011. At WD 6, the majority of the tracers indicated that recharge arrived between 2005 and 2009. A lthough CFC and $\mathrm{SF}_{6}$ concentrations were still rising at WD 6 in 2012, this could partially reflect their rising concentrations in the reservoir in recent years. It is not clear whether reservoir recharge was beginning to reach WD 8, WD 12, or WD 4 during 2012. All three of these wells showed some changes in field parameters (TDG pressure, dissolved oxygen, or specific conductance) and various other environmental tracers, but no increase in $\mathrm{Cl}: \mathrm{Br}$ ratios was observed during 2012. While some tracers sampled at WD 15 and WD 19 were elevated above background levels, this could have been caused by the rising water table rather than the arrival of reservoir recharge. This interpretation is consistent with the fact that the wells are farther from the reservoir and had low $\mathrm{Cl}: \mathrm{Br}$ ratios. This is also supported by environmental tracer concentrations at monitoring wells WD 16 and WD 20, deeper monitoring wells at the same sites as WD 15 and WD 19, respectively, that showed no indication of the arrival of reservoir recharge through 2012. M onitoring wells WD 5, WD RJ, and WD 18, similarly, did not show any indication of the arrival of reservoir recharge by 2012. 


\section{Assessment of Managed Aquifer Recharge at Sand Hollow Reservoir, Updated to Conditions in 2012}

Table 4. Summary of tracer peaks showing year of arrival for reservoir recharge at selected monitoring wells in Sand Hollow, Utah. [Cl:Br, chloride-to-bromide ratio; - , no data available; ?, uncertain year of tracer peak; NMF, not meaningful]

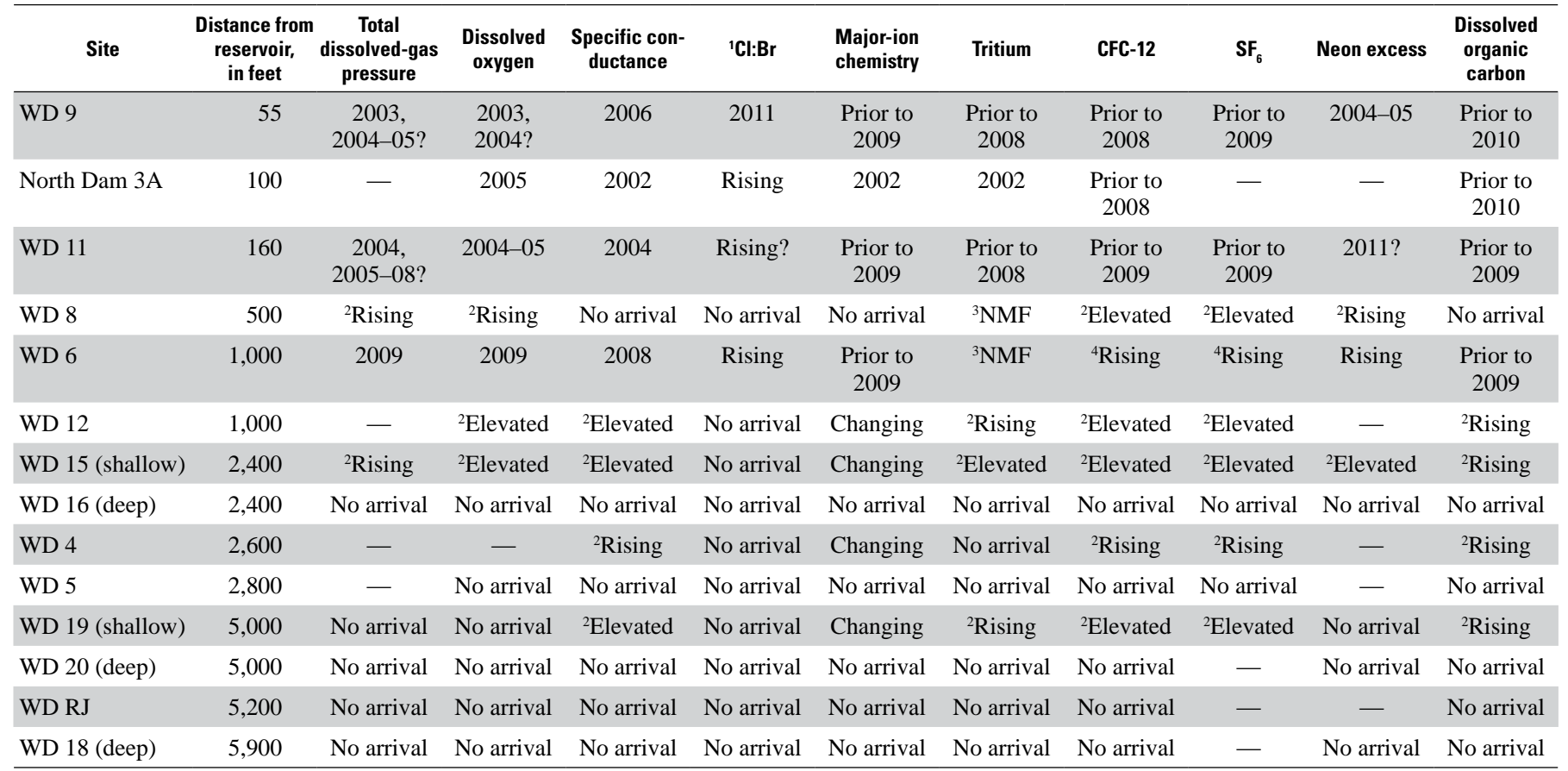

${ }^{1}$ B ased on data from both Table 3 of this report and Table 1 of Heilweil and others (2009).

${ }^{2}$ Rising or elevated dissolved-gas pressure, dissolved oxygen, specific conductance, tritium, CFC-12, SF 6 , neon excess, or dissolved organic carbon during 2011-2012 may indicate local natural recharge and rising water table (dissolution of trapped air bubbles and chloride bulge) rather than reservoir recharge.

${ }^{3} \mathrm{~N}$ ot meaningful because of elevated tritium concentrations prior to reservoir construction.

${ }^{4}$ Rising CFC-12 and (or) SF ${ }_{6}$ concentrations in the reservoir during 2008-2012 complicate the interpretation of peak arrival year. 


\section{Summary}

The objectives of this study were to both quantify amounts of recharge from Sand Hollow Reservoir and to evaluate its movement through the Navajo Sandstone aquifer, with conditions updated to 2012. This study follows previous USG S Scientific Investigations Reports 2005-5185 (Heilweil and others, 2005), 2007-5023 (Heilweil and Susong, 2007), 2009-5050

(Heilweil and others, 2009), and Heilweil and M arston (2011).

Since its inception in 2002, diversions to the reservoir from the nearby Virgin River have resulted in generally rising reservoir altitude, from about $3,000 \mathrm{ft}$ in 2002 to a maximum of about $3,060 \mathrm{ft}$ in 2006 , which then fluctuated between about 3,040 and 3,060 ft from 2008 through 2011. Similarly, groundwater levels in monitoring wells closest to the reservoir generally rose between 2002 and 2006, and then fluctuated with reservoir altitude and pumpage rate from nearby production wells. Water levels in monitoring wells farther from the reservoir were still rising during 2011.

A bout 19,000 acre-ft of groundwater were withdrawn from Sand Hollow Reservoir between 2004 and 2011, mostly from production wells located near the N orth Dam. French drains, installed to capture shallow seepage near the reservoir, are also pumped as they fill with water. About 6,000 acre-ft of groundwater were pumped from the N orth D am drain between 2003 and 2011. This water initially was returned to the reservoir, but since 2007 has been used by Sand Hollow Resort for irrigation. A bout 1,500 acre-ft of water were pumped from the West Dam drain back into the reservoir from 2005 through 2011. In 2006, the West Dam Spring drain was constructed and has largely replaced the function of the West Dam drain. From 2006 through 2011 about 13,500 acre-ft have been pumped from this drain into the Washington County Water Conservancy District's municipal supply system.

Total annual surface-water inflow to Sand Hollow Reservoir ranged from about 800 acre-ft in 2007 to 56,000 acre-ft in 2005. Total inflow from 2002 through 2011 was about 199,000 acre-ft. The general increase in reservoir water-level altitude and surface area from 2002 and 2007 resulted in a steady increase in the volume of annual evaporation from about 1,000 in 2002 to about 6,600 acre-ft through 2006, which leveled off from 2007 through 2011. Total estimated cumulative evaporative loss from 2002 through 2009 was about 50,000 acre-ft. During this same period, annual reservoir recharge to the underlying Navajo Sandstone aquifer fluctuated between about 5,000 and 18,000 acre-ft. A nnual recharge for 2010 and 2011was approximately 11,000 and 8,400 acre-ft, respectively. Total calculated reservoir recharge from 2002 through 2011 was about 106,000 acre-ft with a two standard deviation uncertainty of 9,200 acre-ft. From 2002 through 2011, calculated monthly recharge volumes ranged from about 50 to 3,500 acre-ft, and average daily recharge rates (cal culated for each month) ranged from 0.001 to $0.43 \mathrm{ft}$. From M arch 2002 through M ay 2002, there was a rapid decrease in rates as the vadose zone wetted up and the reservoir became hydraulically connected to the aquifer. From mid-2002 through 2007, there was a gentler decline in recharge rates, likely caused by both the decreasing hydraulic gradient in the aquifer and permeability reduction beneath the reservoir. From 2007 through 2011, recharge rates generally remained low but were highly variable from month to month.

Water quality was assessed at various monitoring wells in Sand Hollow to evaluate the timing and location of reservoir recharge moving through the aquifer. Tracers of reservoir recharge include major and minor dissolved inorganic ions, tritium, dissolved organic carbon, and dissolved industrial and noble gases, including chlorofluorocarbons, sulfur hexafluoride, and neon. The various tracers often have different peak arrival years at individual monitoring wells. This is likely due to different behavioral characteristics of each of the tracers, such as adsorption and retardation, dispersion, and gas dissolution as recharge enters and moves through the aquifer. By 2012, reservoir recharge clearly arrived at four monitoring wells (North Dam 3A, W D 6, W D 9, WD 11) within about $1,000 \mathrm{ft}$ of the reservoir. Tracer concentrations at five other monitoring wells (WD 5, WD RJ, WD 16, WD 18, WD 20) indicated that reservoir recharge had not reached these sites. The low $\mathrm{Cl}: \mathrm{Br}$ ratios but elevated or increasing environmental tracer concentrations at the remaining monitoring wells (WD 4, WD 8, WD 12, WD 15, WD 19), are difficult to interpret; these changes could either be an early indication of the arrival of reservoir recharge or could be caused by other processes such as local mobilization of vadose-zone salts and incorporation of dissolved gases associated with rising groundwater levels. 


\section{References Cited}

A eschbach-H ertig, W., Peeters, F., B eyerle, U., and Kipfer, R.,2000, Pal eotemperature reconstruction from noble gases in ground water taking into account equilibrium with trapped air: Nature, v. 405, p. 1040-1044.

Clarke, W.B., J enkins, W.J ., and Top, Z., 1976, D etermination of tritium by mass spectrometric measurements of ${ }^{3} \mathrm{He}$ : International J ournal of A pplied Radiation Isotopes, v. 27, p. 515-522.

Freeze, R.A ., and Cherry, J.A ., 1979, Groundwater: PrenticeHall, Inc., Englewood Cliffs, N ew J ersey, 604 p.

Gardner, P., and Solomon, D.K., 2009, An advanced passive diffusion sampler for the determination of dissolved gas concentrations: Water Resources Research, v. 45, W 06423.

Heilweil, V.M ., Freethey, G.W., Stolp, B.J ., Wilkowske, C.D., and Wilberg, D.E., 2000, Geohydrology and numerical simulation of ground-water flow in the central Virgin River basin of Iron and Washington Counties, U tah: U tah Department of Natural Resources Technical Publication 116, $182 \mathrm{p}$.

Heilweil, V.M ., and M arston, T.M ., 2011, A ssessment of managed aquifer recharge from Sand Hollow Reservoir, Washington County, updated to conditions in 2010: U.S. Geological Survey Scientific Investigations Report 2011$5142,39 \mathrm{p}$.

Heilweil, V.M., and McKinney, T.S., 2007, Net-infiltration map of the Navajo Sandstone outcrop area in western Washington County, Utah: U.S. Geological Survey Scientific Investigations M ap 2988.

Heilweil, V.M., M cK inney, T.S., Zhdanov M.S., and Watt, D.E., 2007, Controls on the variability of net infiltration to desert sandstone: Water Resources Research, v. 43, W 07431. D OI:10.1029/2006W R 005113, 15 p.

Heilweil, V.M ., Ortiz, G., and Susong, D.D., 2009a, A ssessment of managed aquifer recharge at Sand Hollow Reservoir, Washington County, U tah, updated to conditions through 2007: U.S. Geological Survey Scientific Investigations Report 2009-5050, 19 p.

Heilweil, V.M . and Solomon, D.K ., 2004, M illimeter- to kilometer-scale variations in vadose-zone bedrock solutes: implications for estimating recharge in arid settings, in Phillips, F., Scanlon, B ., and Hogan, J., eds., Ground-water recharge in a desert environment: the southwestern $U$ nited States, Water Science and A pplication 9: Washington, D.C., A merican Geophysical Union, p. 49-67.
Heilweil, V.M ., Solomon, D.K ., and Gardner, P.M ., 2006, Borehole environmental tracers for evaluating net infiltration and recharge through desert bedrock: Vadose Zone Journal, v. 5, p. 98-120.

Heilweil, V.M., Solomon, D.K ., and Ortiz, G., 2009b, Silt and gas accumulation beneath an artificial recharge spreading basin, southwestern U tah, U.S.A: B ol etin Geologico y M inero, v. 120, no. 2, p. 185-195.

Heilweil, V.M ., Solomon, D.K., Perkins, K.S., and Ellett, K.M ., 2004, Gas-partitioning tracer test to quantify trapped gas during recharge: Ground Water, v. 42, no. 4, p. 589-600.

Heilweil, V.M ., and Susong, D.D., 2007, A ssessment of artificial recharge at Sand Hollow Reservoir, Washington County, U tah, updated to conditions through 2006: U.S. Geological Survey Scientific Investigations Report 20075023, $14 \mathrm{p}$.

Heilweil, V.M ., Susong, D.D., Gardner, P.M ., and Watt, D.E, 2005, Pre- and post-reservoir ground-water conditions and assessment of artificial recharge at Sand Hollow, Washington County, U tah, 1995-2005: U.S. Geological Survey Scientific Investigations Report 2005-5185, 74 p.

Heilweil, V.M., and Watt, D.E., 2011, Trench infiltration for managed aquifer recharge to permeable bedrock: Hydrological Processes 25, DOI: 10.1002/hyp.7833, p. 141-151.

Hurlow, H.A ., 1998, The geology of the central Virgin River basin, southwestern $U$ tah, and its relation to ground-water conditions: State of U tah Water Resources Bulletin 26, 53 p.

M arston, T.M ., and Heilweil, V.M ., 2012, Numerical simulation of groundwater movement and managed aquifer recharge from Sand Hollow R eservoir, Hurricane B ench area, Washington County, U tah: U.S. Geological Survey Scientific Investigations Report 2012-5236, 34 p.

M cGuinness, J.L., and Bordne, E.F., 1971, A comparison of lysimeter-derived potential evapotranspiration with computed values: U.S. D epartment of A griculture Technical Bulletin 1472, A gricultural Research Service, Washington D.C., 71 p.

Rosenberry, D.O., Winter, T.C., B uso, D.C., and Likens, G.E., 2007, Comparison of 15 evaporation methods applied to a small mountain lake in the northeastern USA : J ournal of Hydrology, v. 340, p. 149-166.

Sheldon, A ., 2002, Diffusion of radiogenic helium in shallow ground water: Implications for crustal degassing: Salt Lake City, U tah, U niversity of U tah, Ph.D. Dissertation, 185 p.

Wilde, F.D., and Radtke, D.B., 1998, National field manual for the collection of water-quality data: Field measurements: U.S. Geological Survey Techniques of Water-Resources Investigations, book 9, chap. A6, 233 p. 

